\title{
Investigation of a Flexible Enantiospecific Approach to Aziridines
}

\author{
Clare E. Jamookeeah, ${ }^{\dagger}$ Christopher D. Beadle, ${ }^{\dagger}$ Richard F. W. Jackson ${ }^{\dagger}$ \\ and Joseph P. A. Harrity ${ }^{*, \dagger}$ \\ ${ }^{\dagger}$ Department of Chemistry, University of Sheffield, Sheffield, S3 7HF, U. K. \\ Lilly UK, Erl Wood Manor, Windlesham, Surrey, GU20 6PH, U. K.
}

\section{Table of Contents}

E-mail: j.harrity@sheffield.ac.uk

General Information

Supporting Information

Preparation of $\mathbf{1 1}$

Preparation of $\mathbf{1 2}$

Preparation of $\mathbf{1 3}$

Preparation of $\mathbf{1 4}$

Preparation of $\mathbf{1 5}$

Preparation of $\mathbf{1 6}$

Preparation of $\mathbf{2 1}$

Preparation of $\mathbf{2 2}$

Preparation of $\mathbf{2 3}$

Preparation of $\mathbf{2 4}$

Preparation of $\mathbf{2 5}$

Preparation of $\mathbf{2 6}$

Preparation of $\mathbf{2 7}$

${ }^{1} \mathrm{H} /{ }^{13} \mathrm{C}$ NMR Spectrum of $\mathbf{2}$

${ }^{1} \mathrm{H} /{ }^{13} \mathrm{C}$ NMR Spectrum of $\mathbf{1 0}$

${ }^{1} \mathrm{H} /{ }^{13} \mathrm{C}$ NMR Spectrum of $\mathbf{1 1}$

${ }^{1} \mathrm{H} /{ }^{13} \mathrm{C}$ NMR Spectrum of $\mathbf{1 2}$

${ }^{1} \mathrm{H} /{ }^{13} \mathrm{C}$ NMR Spectrum of $\mathbf{1 3}$

${ }^{1} \mathrm{H} /{ }^{13} \mathrm{C}$ NMR Spectrum of $\mathbf{1 4}$

${ }^{1} \mathrm{H} /{ }^{13} \mathrm{C}$ NMR Spectrum of $\mathbf{1 5}$

${ }^{1} \mathrm{H} /{ }^{13} \mathrm{C}$ NMR Spectrum of $\mathbf{1 6}$

${ }^{1} \mathrm{H} /{ }^{13} \mathrm{C}$ NMR Spectrum of $\mathbf{1 7}$

${ }^{1} \mathrm{H} /{ }^{13} \mathrm{C}$ NMR Spectrum of $\mathbf{2 0}$ 
${ }^{1} \mathrm{H} /{ }^{13} \mathrm{C}$ NMR Spectrum of $\mathbf{2 4}$

${ }^{1} \mathrm{H} /{ }^{13} \mathrm{C}$ NMR Spectrum of $\mathbf{2 5}$

${ }^{1} \mathrm{H} /{ }^{13} \mathrm{C}$ NMR Spectrum of $\mathbf{2 6}$

${ }^{1} \mathrm{H} /{ }^{13} \mathrm{C}$ NMR Spectrum of $\mathbf{2 7}$

HPLC Trace of $(R)-\mathbf{2 0}$

26

HPLC Trace of racemic $\mathbf{2 0}$

27

X-ray data of $\mathbf{2 5}$

28

X-ray data of $\mathbf{2 6}$

49

X-ray data of $\mathbf{2 7}$

59 


\section{General Information}

All reactions were conducted in oven or flame-dried glassware under an inert atmosphere of dry nitrogen. Flash chromatography was performed on silica gel (BDH Silica Gel 60 43-60, or Fluorochem Davisil silica gel 43-60). The solvent system used was a gradient of petroleum ether/ ethyl acetate (90-10), increasing in polarity to ethyl acetate. Thin layer chromatography (TLC) was performed on aluminium backed plates pre-coated with silica (0.2 mm, Merck DC-alufolien Kieselgel 60 F254), which were developed using standard visualizing agents: Ultraviolet light or potassium permanganate. ${ }^{1} \mathrm{H} /{ }^{13} \mathrm{C}$ NMR spectra were recorded on Bruker AC-250 or Av1-250 instruments or AMX-400 or AV1400 instruments. ${ }^{1} \mathrm{H}$ : Chemical shifts are reported in ppm with the solvent resonance as the internal standard $\left(\mathrm{CHCl}_{3}: \delta 7.27 \mathrm{ppm}\right)$. Data are reported as follows: chemical shift, multiplicity ( $\mathrm{s}=$ singlet, $\mathrm{d}=$ doublet, $\mathrm{t}=$ triplet, $\mathrm{q}=$ quartet, $\mathrm{br}=\mathrm{broad}, \mathrm{m}=$ =multiplet), integration, coupling constants $(J)$ in Hz. ${ }^{13} \mathrm{C}$ NMR spectra were with complete proton decoupling. Chemical shifts are reported in ppm with the solvent resonance as the internal standard $\left(\mathrm{CDCl}_{3}: \delta 77.0 \mathrm{ppm}\right)$. Infrared (FTIR) spectra were recorded on a Perkin Elmer Paragon 100 FTIR spectrophotometer, $v_{\max }$ in $\mathrm{cm}^{-1}$. Bands are characterized as broad (br), strong (s), medium (m) and weak (w). Samples were recorded as thin films using sodium chloride plates, as a $\mathrm{CH}_{2} \mathrm{Cl}_{2}$ solution or as a $\mathrm{KBr}$ disc. Low resolution mass spectra were recorded on Micromass Autospec, operating in E.I., C.I. or FAB mode; or a Perkin-Elmer Turbomass Bench top GC-MS operating in either E.I. or C.I mode. Highresolution mass spectra (HRMS) recorded for accurate mass analysis, were performed on either a MicroMass LCT operating in Electrospray mode (TOF ES+) or a MicroMass Prospec operating in either FAB (FAB+), EI (EI+) or CI (CI+) mode. Melting points were performed on recrystallized solids and recorded on a Gallenkamp melting point apparatus and are uncorrected. All solvents and reagents were purified using standard, laboratory techniques according to methods published in "Purification of Laboratory Chemicals" by Perrin, Armarego, and Perrin (Pergamon Press, 1966). 
(R)-2-(Pent-4-enyl)-1-tritylaziridine 11. To a solution of $2(0.1 \mathrm{~g}, 0.213 \mathrm{mmol}, 1 \mathrm{eq})$, CuBr.DMS (11 mg, $0.05 \mathrm{mmol}, 0.25 \mathrm{eq})$ in THF (1.3 mL) was added but-3enylmagnesium bromide solution in THF $(0.44 \mathrm{M}, 0.72 \mathrm{~mL}, 0.426 \mathrm{mmol}, 2 \mathrm{eq})$. Flash chromatography (5:1, petroleum ether: ethyl acetate) provided 11 as a clear oil $(67 \mathrm{mg}$, $89 \%) .[\alpha]^{22}-23.3\left(c 3.00, \mathrm{CHCl}_{3}\right) .{ }^{1} \mathrm{H}$ NMR $\left(250 \mathrm{MHz}, \mathrm{CDCl}_{3}\right): \delta 1.05(1 \mathrm{H}, \mathrm{d}, J=6.0$ $\mathrm{Hz}), 1.18(1 \mathrm{H}, \mathrm{m}), 1.43(3 \mathrm{H}, \mathrm{m}), 1.58(1 \mathrm{H}, \mathrm{d}, J=3.0 \mathrm{~Hz}), 1.89-2.08(3 \mathrm{H}, \mathrm{m}), 4.92-5.02$ $(2 \mathrm{H}, \mathrm{m}), 5.71-5.87(1 \mathrm{H}, \mathrm{m}), 7.18-7.53(15 \mathrm{H}, \mathrm{m}) ;{ }^{13} \mathrm{C} \mathrm{NMR}\left(62.9 \mathrm{MHz}, \mathrm{CDCl}_{3}\right): \delta 26.3$, 26.5, 32.2, 32.8, 33.7, 74.1, 107.4, 114.5, 126.5, 127.3, 129.6, 144.9; FTIR (film, $v_{\max }$ $\left.\mathrm{cm}^{-1}\right) 3056(\mathrm{w}), 2927$ (w), 1596 (s), 1489 (s), 1447 (m); HRMS (FAB) m/z $\left(\mathrm{MH}^{+}\right)$calcd for $\mathrm{C}_{26} \mathrm{H}_{28} \mathrm{~N}$ 354.2221, found 354.2214.

(R)-2-(But-3-enyl)-1-tritylaziridine 12. To a solution of $2(0.15 \mathrm{~g}, 0.31 \mathrm{mmol}, 1 \mathrm{eq})$, CuBr.DMS (16 mg, $0.08 \mathrm{mmol}, 0.25 \mathrm{eq})$ in THF (2.0 mL) was allylmagnesium bromide solution in diethyl ether $(0.50 \mathrm{M}, 1.3 \mathrm{~mL}, 0.64 \mathrm{mmol}, 2 \mathrm{eq})$. Flash chromatography on $\mathrm{AgNO}_{3} 10 \%$ silica, (1:1 ethyl acetate: petroleum ether) provided a mixture of $\mathbf{2}$ and $\mathbf{1 2}$, $0.06 \mathrm{~g}$. Flash chromatography (5:1 petroleum ether: EtOAc) provided $\mathbf{1 2}$ as a clear oil (39 $\mathrm{mg}, 36 \%) .[\alpha]_{\mathrm{D}}^{22}-22.0\left(\right.$ c 2.20, $\left.\mathrm{CHCl}_{3}\right) .{ }^{1} \mathrm{H} \mathrm{NMR}\left(250 \mathrm{MHz}, \mathrm{CDCl}_{3}\right): \delta 0.97(1 \mathrm{H}, \mathrm{d}$, $J=6.0 \mathrm{~Hz}), 1.12(1 \mathrm{H}, \mathrm{m}), 1.46(1 \mathrm{H}, \mathrm{m}), 1.51(1 \mathrm{H}, \mathrm{d}, J=3.0 \mathrm{~Hz}), 1.86(1 \mathrm{H}, \mathrm{m}), 1.98(2 \mathrm{H}$, $\mathrm{m})$, 4.79-4.87 (2H, m), $5.71(1 \mathrm{H}, \mathrm{m}), 7.16-7.44(15 \mathrm{H}, \mathrm{m}) ;{ }^{13} \mathrm{C} \mathrm{NMR}\left(62.9 \mathrm{MHz}, \mathrm{CDCl}_{3}\right)$ :

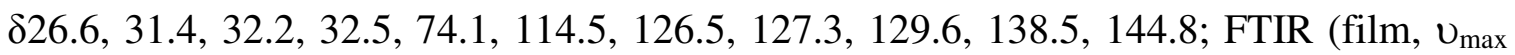
$\left.\mathrm{cm}^{-1}\right) 3051(\mathrm{w}), 2934(\mathrm{w}), 1482$ (s), 1445 (s); HRMS (CI) $\mathrm{m} / z\left(\mathrm{M}^{+}\right)$calcd for $\mathrm{C}_{25} \mathrm{H}_{25} \mathrm{~N}$ 339.1987, found 339.1976.

(R)-2-Benzyl-1-tritylaziridine 13. To a solution of $2(0.2 \mathrm{~g}, 0.43 \mathrm{mmol}, 1 \mathrm{eq})$, CuBr.DMS (22 mg, $0.11 \mathrm{mmol}, 0.25 \mathrm{eq})$ in THF (2.6 mL) was added phenylmagnesium bromide solution in THF (0.39M, $3.3 \mathrm{~mL}, 1.28 \mathrm{mmol}, 2 \mathrm{eq})$. Flash chromatography (20:1, petroleum ether: EtOAc) provided 13 as a colourless solid upon trituration with $\mathrm{MeOH}$ (0.22 g, $90 \%)$. Mp 75-78 ${ }^{\circ} \mathrm{C} .[\alpha]^{24} \mathrm{D}-47.6$ (c 1.00, $\left.\mathrm{CHCl}_{3}\right) .{ }^{1} \mathrm{H}$ NMR (250 MHz, $\left.\mathrm{CDCl}_{3}\right)$ : $\delta 1.11(1 \mathrm{H}, \mathrm{d}, J=6.0 \mathrm{~Hz}), 1.48(1 \mathrm{H}, \mathrm{m}), 1.72(1 \mathrm{H}, \mathrm{d}, J=3.0 \mathrm{~Hz}), 2.68(1 \mathrm{H}, \mathrm{dd}, J=14.0,8.0$ $\mathrm{Hz}), 3.28(1 \mathrm{H}, \mathrm{dd}, J=14.0,4.5 \mathrm{~Hz}), 7.18-7.35(14 \mathrm{H}, \mathrm{m}), 7.57(6 \mathrm{H}, \mathrm{d}, J=7.0 \mathrm{~Hz}) ;{ }^{13} \mathrm{C}$ NMR $\left(62.9 \mathrm{MHz}, \mathrm{CDCl}_{3}\right)$ : $\delta 27.3,34.0,39.5,74.4,126.4,126.8,127.7,128.5,129.1$, 129.8, 139.8, 145.0 ; FTIR (film, $\mathrm{v}_{\max } \mathrm{cm}^{-1}$ ) 3259 (w), 1620 (s); HRMS (EI) $\mathrm{m} / z$ calcd for $\mathrm{C}_{28} \mathrm{H}_{25} \mathrm{~N} 375.1987$, found 375.1981.

(R)-2-(4-Chlorobenzyl)-1-tritylaziridine 14. To a solution of $2(0.2 \mathrm{~g}, 0.43 \mathrm{mmol}, 1$ eq), CuBr.DMS (22 mg, $0.11 \mathrm{mmol}, 0.25 \mathrm{eq}$ ) in THF (1.3 mL) was added (4chlorophenyl)magnesium bromide solution in THF (0.2M, $4.3 \mathrm{~mL}, 0.85 \mathrm{mmol}, 2 \mathrm{eq})$. Flash chromatography (20:1, petroleum ether: EtOAc) provided $\mathbf{1 4}$ as a colourless solid after trituration with $\mathrm{MeOH}(0.11 \mathrm{~g}, 65 \%)$. Mp 74-76 ${ }^{\circ} \mathrm{C}$. $[\alpha]^{22} \mathrm{D}-50.0\left(c 1.00, \mathrm{CHCl}_{3}\right)$. ${ }^{1} \mathrm{H}$ NMR (250 MHz, $\left.\mathrm{CDCl}_{3}\right): \delta 1.02(1 \mathrm{H}, \mathrm{d}, J=6.0 \mathrm{~Hz}), 1.37(1 \mathrm{H}, \mathrm{m}), 1.59(1 \mathrm{H}, \mathrm{d}, J=3.0$ 
$\mathrm{Hz}), 2.63(1 \mathrm{H}, \mathrm{dd}, J=14.0,7.5 \mathrm{~Hz}), 3.12(1 \mathrm{H}, \mathrm{dd}, J=14.0,4.5 \mathrm{~Hz}), 7.13(2 \mathrm{H}, \mathrm{d}, J=8.5 \mathrm{~Hz})$, 7.22-7.35 (11H, m), 7.53-7.58 (6H, m). $\left.{ }^{13} \mathrm{C} \mathrm{NMR} \mathrm{(62.9} \mathrm{MHz,} \mathrm{CDCl}_{3}\right): \delta 26.8,33.5,38.5$, 74.1, 126.6, 127.4, 128.4, 129.5, 130.1, 131.9, 137.9, 144.6; FTIR (film, $v_{\max } \mathrm{cm}^{-1}$ ) 2920 (w), $1597(\mathrm{w}), 1490(\mathrm{~m}), 1447$ (m); HRMS (EI) $\mathrm{m} / \mathrm{z}\left(\mathrm{M}^{+}\right)$calcd for $\mathrm{C}_{28} \mathrm{H}_{24} \mathrm{~N}^{35} \mathrm{Cl}$ 409.1597, found 409.1583.

(R)-2-(3-(1,3-Dioxolan-2-yl)propyl)-1-tritylaziridine 15. To a solution of 2 (0.1 g, $0.213 \mathrm{mmol}, 1 \mathrm{eq}), \mathrm{CuBr} . \mathrm{DMS}$ (22 $\mathrm{mg}, 0.1 \mathrm{mmol}, 0.5 \mathrm{eq})$ in THF (1.3 mL) was added [2(1,3-dioxolan-2-yl)ethyl]magnesium bromide in THF $(0.40 \mathrm{M}, 1.1 \mathrm{~mL}, 0.426 \mathrm{mmol}, 2$ eq). Flash chromatography (6:1, petroleum ether: EtOAc) provided $\mathbf{1 5}$ as a clear oil (69 mg, $81 \%$ ). $[\alpha]^{22}{ }_{\mathrm{D}}-20.0\left(c\right.$ 1.00, $\left.\mathrm{CHCl}_{3}\right) .{ }^{1} \mathrm{H}$ NMR $\left(250 \mathrm{MHz}, \mathrm{CDCl}_{3}\right): \delta 1.04(1 \mathrm{H}, \mathrm{d}$, $J=6.0 \mathrm{~Hz}), 1.17(1 \mathrm{H}, \mathrm{m}), 1.36-1.49(3 \mathrm{H}, \mathrm{m}), 1.57-1.68(3 \mathrm{H}, \mathrm{m}), 1.96(1 \mathrm{H}, \mathrm{m}), 3.79-3.97$ $(4 \mathrm{H}, \mathrm{m}), 4.81(1 \mathrm{H}, \mathrm{t}, J=4.5 \mathrm{~Hz}), 7.15-7.33(9 \mathrm{H}, \mathrm{m}), 7.45-7.55(6 \mathrm{H}, \mathrm{m}) ;{ }^{13} \mathrm{C}$ NMR $(62.9$ $\left.\mathrm{MHz}, \mathrm{CDCl}_{3}\right): \delta 21.6,25.6,32.6,32.7,33.7,64.8,74.0,104.5,126.5,127.3,129.5$, 144.9; FTIR (film, $v_{\max } \mathrm{cm}^{-1}$ ) 3056 (w), 1596 (w), 1489 (m), 1447 (m); HRMS (EI) $\mathrm{m} / z$ $\left(\mathrm{M}^{+}\right)$calcd for $\mathrm{C}_{27} \mathrm{H}_{29} \mathrm{NO}_{2} 399.2198$, found 399.2195

(R)-2-(2-(Trimethylsilyl)allyl)-1-tritylaziridine 16. To a solution of 2 (0.05 g, 0.11 mmol, 1 eq), CuBr.DMS ( $6 \mathrm{mg}, 0.03 \mathrm{mmol}, 0.25 \mathrm{eq}$ ) in THF (0.7 mL) was added 1trimethylsilyl vinylmagnesium bromide solution in THF $(0.30 \mathrm{M}, 0.76 \mathrm{~mL}, 0.212 \mathrm{mmol}, 2$ eq). Flash chromatography (6:1, petroleum ether: EtOAc) provided $\mathbf{1 6}$ as a clear oil (36 $\mathrm{mg}, 82 \%) .[\alpha]^{22}{ }_{\mathrm{D}}-20.1\left(c 1.50, \mathrm{CHCl}_{3}\right) .{ }^{1} \mathrm{H}$ NMR $\left(250 \mathrm{MHz}, \mathrm{CDCl}_{3}\right): \delta 0.00(9 \mathrm{H}, \mathrm{s}$, $\left.\mathrm{Si}\left(\mathrm{CH}_{3}\right)_{3}\right), 1.12(1 \mathrm{H}, \mathrm{d}, J=6.0 \mathrm{~Hz}), 1.26-1.36(1 \mathrm{H}, \mathrm{m}), 1.58(1 \mathrm{H}, \mathrm{d}, J=3.0 \mathrm{~Hz}), 2.13(1 \mathrm{H}$, dd, $J=15.0,8.5 \mathrm{~Hz}), 2.89(1 \mathrm{H}, \mathrm{dd}, J=15.03 .5 \mathrm{~Hz}), 5.26-5.27(1 \mathrm{H}, \mathrm{m}), 5.50-5.53(1 \mathrm{H}$, m), 7.16-7.32 (9H, m), 7.45-7.54 (6H, m); ${ }^{13} \mathrm{C}$ NMR (62.9 $\left.\mathrm{MHz}, \mathrm{CDCl}_{3}\right): \delta 0.0,29.1$, 34.1, 41.0, 75.8, 126.7, 128.2, 129.0, 131.2, 146.5, 151.3; FTIR (film, $v_{\max } \mathrm{cm}^{-1}$ ) 3056 (m), 2955 (m), 1683 (m), 1608 (w), 1489 (m); HRMS (TOF ESI+) calcd. for $\mathrm{C}_{27} \mathrm{H}_{32} \mathrm{NSi}$ (MH+): 398.2304, found: 398.2304

(R)-2-Benzyl-1-(4-methoxyphenylsulfonyl)aziridine 21. To a solution of $13(0.1 \mathrm{~g}$, $0.27 \mathrm{mmol}, 1 \mathrm{eq})$ in a mixture of chloroform $(1.5 \mathrm{~mL})$ and methanol $(0.5 \mathrm{~mL})$ was added trifluoroacetic acid $(0.616 \mathrm{~mL}, 7.99 \mathrm{mmol}, 30 \mathrm{eq})$ at $-78{ }^{\circ} \mathrm{C}$. The solution was stirred at $-78{ }^{\circ} \mathrm{C}$ for 15 minutes then, allowed to warm to $0{ }^{\circ} \mathrm{C}$ for 4 hours upon which triethylamine $(1.23 \mathrm{~mL}, 8.79 \mathrm{mmol}, 33 \mathrm{eq})$ was added to the solution at $0{ }^{\circ} \mathrm{C}$. After 10 mins, 4-methoxybenzene-1-sulfonyl chloride (70 mg, $0.32 \mathrm{mmol}, 1.2 \mathrm{eq}$ ) was added and the mixture was stirred overnight at $0{ }^{\circ} \mathrm{C} . \mathrm{NaHCO}_{3}$ solution was added and the solution was extracted with chloroform. The organic extracts were dried over $\mathrm{MgSO}_{4}$ and concentrated in vacuo and the product purified by flash chromatography (5:1 petroleum ether: EtOAc) to provide 21 as a pale yellow solid (65 mg, 79\%). Mp 81-83 ${ }^{\circ} \mathrm{C}$. $[\alpha]^{22} \mathrm{D}-$ 
6.3 (c 1.60, $\left.\mathrm{CHCl}_{3}\right) ; \mathrm{Lit}^{1}(\mathrm{~S})-21:[\alpha]_{\mathrm{D}}^{25}=+9.6\left(c\right.$ 1.02, $\left.\mathrm{CHCl}_{3}\right) .{ }^{1} \mathrm{H}$ NMR $(250 \mathrm{MHz}$, $\left.\mathrm{CDCl}_{3}\right): \delta 2.15(1 \mathrm{H}, \mathrm{d}, J=2.0 \mathrm{~Hz}), 2.63-2.70(3 \mathrm{H}, \mathrm{m}), 2.91(1 \mathrm{H}, \mathrm{m}), 3.86(3 \mathrm{H}, \mathrm{s}), 6.85$ $(2 \mathrm{H}, \mathrm{d}, J=9.0 \mathrm{~Hz}), 7.00-7.16(5 \mathrm{H}, \mathrm{m}), 7.70(2 \mathrm{H}, \mathrm{d}, J=9.0 \mathrm{~Hz}) ;{ }^{13} \mathrm{C} \mathrm{NMR}(62.9 \mathrm{MHz}$, $\left.\mathrm{CDCl}_{3}\right): \delta 32.7,37.5,41.2,55.6,114.2,126.6,128.5,128.7,129.3,130.0,137.1,163.5$; FTIR (film, $v_{\max } \mathrm{cm}^{-1}$ ) 3063 (w), 2921 (w), 1596 (s) 1499 (s); HRMS (TOF ESI+) $\mathrm{m} / \mathrm{z}$ $\left(\mathrm{MNa}^{+}\right)$calcd for $\mathrm{C}_{16} \mathrm{H}_{17} \mathrm{NO}_{3} \mathrm{NaS} 326.0827$, found 326.0813.

(R)-1-(Benzyloxycarbonyl)-2-benzylaziridine 22. To a solution of $13(0.1 \mathrm{~g}, 0.27$ mmol, $1 \mathrm{eq})$ in a mixture of chloroform $(1.5 \mathrm{~mL})$ and methanol $(0.5 \mathrm{~mL})$ was added trifluoroacetic acid $(0.616 \mathrm{~mL}, 7.99 \mathrm{mmol}, 30 \mathrm{eq})$ at $-78{ }^{\circ} \mathrm{C}$. The solution was stirred at $-78{ }^{\circ} \mathrm{C}$ for 15 minutes then, allowed to warm to $0{ }^{\circ} \mathrm{C}$ for 4 hours upon which triethylamine $(1.23 \mathrm{~mL}, 8.79 \mathrm{mmol}, 33 \mathrm{eq})$ was added to the solution at $0{ }^{\circ} \mathrm{C}$. After 10 mins, benzyl chloroformate $(0.046 \mathrm{~mL}, 0.32 \mathrm{mmol}, 1.2 \mathrm{eq})$ was added and the mixture was stirred overnight at $0{ }^{\circ} \mathrm{C}$. $\mathrm{NaHCO}_{3}$ solution was added and the solution was extracted with chloroform. The organic extracts were dried over $\mathrm{MgSO}_{4}$ and concentrated in vacuo and the product purified by flash chromatography (7:1 petroleum ether: EtOAc) to provide 22 as a clear oil $(61 \mathrm{mg}, 85 \%)$. $[\alpha]^{22}{ }_{\mathrm{D}}-38.9\left(c 1.80, \mathrm{CHCl}_{3}\right) .{ }^{1} \mathrm{H}$ NMR $(250 \mathrm{MHz}$, $\left.\mathrm{CDCl}_{3}\right): \delta 2.10(1 \mathrm{H}, \mathrm{d}, J=3.0 \mathrm{~Hz}), 2.38(1 \mathrm{H}, \mathrm{d}, J=6.0 \mathrm{~Hz}), 2.70(2 \mathrm{H}, \mathrm{m}), 2.96(1 \mathrm{H}, \mathrm{m})$, $5.11(2 \mathrm{H}, \mathrm{s}), 7.25-7.32(10 \mathrm{H}, \mathrm{m}) ;{ }^{13} \mathrm{C} \mathrm{NMR}\left(62.9 \mathrm{MHz}, \mathrm{CDCl}_{3}\right): \delta 31.6,38.3,38.5,68.1$, 126.6, 128.0, 128.2, 128.5, 128.8, 135.7, 137.6, 163.2; FTIR (film, $\mathrm{U}_{\max } \mathrm{cm}^{-1}$ ) $3030(\mathrm{w})$, 2921 (m), 2851 (w), 1720 (s), 1497 (w); HRMS (TOF ESI+) m/z (MH ${ }^{+}$) calcd for $\mathrm{C}_{17} \mathrm{H}_{18} \mathrm{NO}_{2} 268.1338$, found 268.1329 .

(R)-2-Benzyl-1-(diphenylphosphoryl)aziridine $23{ }^{2}$ To a solution of $\mathbf{1 3}(0.05 \mathrm{~g}, 0.133$ mmol, $1 \mathrm{eq})$ in chloroform $(0.75 \mathrm{~mL})$ was added trifluoroacetic acid $(0.308 \mathrm{~mL}, 3.99$ mmol, 30 eq) at $-78{ }^{\circ} \mathrm{C}$. The solution was stirred at $-78{ }^{\circ} \mathrm{C}$ for 15 minutes then, allowed to warm to $0{ }^{\circ} \mathrm{C}$ for 4 hours upon which triethylamine $(0.613 \mathrm{~mL}, 4.39 \mathrm{mmol}, 33 \mathrm{eq})$ was added to the solution at $0{ }^{\circ} \mathrm{C}$. After 10 mins, diphenylphosphinyl chloride $(0.05 \mathrm{~mL}, 0.27$ mmol, 2 eq) was added and the mixture was stirred overnight at $0{ }^{\circ} \mathrm{C} . \mathrm{NaHCO}_{3}$ solution was added and the solution was extracted with chloroform. The organic extracts were dried over $\mathrm{MgSO}_{4}$ and concentrated in vacuo and the product purified by flash chromatography (1:1 petroleum ether: EtOAc) to provide $\mathbf{2 3}$ as a colourless solid (31 $\mathrm{mg}$, $65 \%) . \mathrm{Mp} 113-115{ }^{\circ} \mathrm{C}\left(\mathrm{lit}=112-114{ }^{\circ} \mathrm{C}\right),[\alpha]^{22} \mathrm{D}-10.0\left(c 1.00, \mathrm{CHCl}_{3}\right) .{ }^{1} \mathrm{H}$ NMR (250 $\left.\mathrm{MHz}_{\mathrm{CDCl}}\right): \delta 1.97(1 \mathrm{H}, \mathrm{ddd}, J=12.0,3.5,1.0 \mathrm{~Hz}), 2.53(1 \mathrm{H}, \mathrm{ddd}, J=17.5,6.0,1.0$ $\mathrm{Hz}), 2.82(2 \mathrm{H}, \mathrm{m}), 2.99(1 \mathrm{H}, \mathrm{m}), 7.06-7.56(11 \mathrm{H}, \mathrm{m}), 7.75-7.95$ (4H, m).

2-(1-Hydroxyethyl)-1-tritylaziridine 24. To a solution of oxalyl chloride $(0.33 \mathrm{ml}, 3.8$ mmol, $1.2 \mathrm{eq})$ in $\mathrm{CH}_{2} \mathrm{Cl}_{2}(27 \mathrm{~mL})$ was added a solution of DMSO (0.56 mL, $7.9 \mathrm{mmol}$,

\footnotetext{
${ }^{1}$ Alonso, A.A.; Andersson, P.G. J. Org. Chem. 1998, 63, 9455.

${ }^{2}$ Cantrill, A. A.; Osborn, M. I.; Sweeney, J. Tetrahedron. 1998, 54, 2181.
} 
$2.5 \mathrm{eq})$ in $\mathrm{CH}_{2} \mathrm{Cl}_{2}(52 \mathrm{~mL})$ at $-78{ }^{\circ} \mathrm{C}$. The solution was stirred for 10 minutes upon which a solution of 1 (1g, $3.17 \mathrm{mmol}, 1 \mathrm{eq})$ in $\mathrm{CH}_{2} \mathrm{Cl}_{2}(26 \mathrm{~mL})$ was added. After one hour triethylamine $(1.86 \mathrm{~mL}, 13.3 \mathrm{mmol}, 4.2 \mathrm{eq})$ was added and the mixture warmed to $\mathrm{rt}$. After one hour the reaction was quenched with water and extracted with $\mathrm{CH}_{2} \mathrm{Cl}_{2}$. The organic extracts were dried over $\mathrm{MgSO}_{4}$ and concentrated in vacuo to provide a crude residue $(1 \mathrm{~g}, 100 \%)$. To a solution of the crude residue $(0.15 \mathrm{~g}, 0.48 \mathrm{mmol}, 1 \mathrm{eq})$ in THF $(3 \mathrm{~mL})$ was added methyllithium $(0.91 \mathrm{~mL}, 0.64 \mathrm{M}, 1.2 \mathrm{eq})$ at $-78{ }^{\circ} \mathrm{C}$. After 30 minutes the solution was warmed to $\mathrm{rt}$ and stirred for an additional 45 minutes. The reaction was quenched with water, washed with brine and extracted with EtOAc. The organic extracts were dried over $\mathrm{MgSO}_{4}$ and concentrated in vacuo. Flash chromatography (4:1 petroleum ether: EtOAc) provided 24 as a colourless solid $(0.14 \mathrm{~g}, 81 \%)$ as a $4: 1$ mixture of diastereoisomers. ${ }^{1} \mathrm{H}$ NMR $\left(250 \mathrm{MHz}, \mathrm{CDCl}_{3}\right): \delta 0.96(0.2 \mathrm{H}, \mathrm{d}, J=6.5 \mathrm{~Hz}), 1.01-1.27$ $(3.8 \mathrm{H}, \mathrm{m}), 1.29-1.34(0.8 \mathrm{H}, \mathrm{m}), 1.39-1.44(0.2 \mathrm{H}, \mathrm{m}), 1.74(0.8 \mathrm{H}, \mathrm{d}, J=3.5 \mathrm{~Hz}), 1.79$ $(0.2 \mathrm{H}, \mathrm{d}, J=4.0 \mathrm{~Hz}), 2.26(0.8 \mathrm{H}, \mathrm{d}, J=5.0 \mathrm{~Hz}), 3.04(0.2 \mathrm{H}, \mathrm{s}), 3.60-3.72(0.8 \mathrm{H}, \mathrm{m}), 4.03-$ $4.11(0.2 \mathrm{H}, \mathrm{m}), 7.20-7.50(15 \mathrm{H}, \mathrm{m}) ;{ }^{13} \mathrm{C} \mathrm{NMR}\left(62.9 \mathrm{MHz}, \mathrm{CDCl}_{3}\right): \delta 19.7,21.0,21.7$, $24.5,37.1,38.8,63.2,68.4,73.7,126.8,126.8,127.6,129.3,129.4,144.2,144.3$, FTIR (film, $v_{\max } \mathrm{cm}^{-1}$ ) 3426 (s), 3045 (w), 2929 (w), 1649 (m), 1490 (m), HRMS (FAB) $\mathrm{m} / \mathrm{z}$ $\left(\mathrm{MH}^{+}\right)$calcd for $\mathrm{C}_{23} \mathrm{H}_{24} \mathrm{NO} 330.1857$, found 330.1846 .

1-Tritylaziridin-2(S)-yl-1(S)-ethyl 4-methylbenzenesulfonate 25. To a solution of 24 (0.125 g, $0.38 \mathrm{mmol}, 1 \mathrm{eq})$ in $\mathrm{CH}_{2} \mathrm{Cl}_{2}(1.9 \mathrm{~mL})$, was added tosyl chloride $(0.08 \mathrm{~g}, 0.42$ mmol, $1.1 \mathrm{eq}$ ), triethylamine ( $58 \mu \mathrm{l}, 0.42 \mathrm{mmol}, 1.1 \mathrm{eq})$ and DMAP ( $5 \mathrm{mg}, 0.037 \mathrm{mmol}$, 0.1 eq) at $0{ }^{\circ} \mathrm{C}$. The solution was left to warm to $\mathrm{rt}$ and stirred for 4 days, washed with $\mathrm{NaHCO}_{3}$ and extracted with $\mathrm{CH}_{2} \mathrm{Cl}_{2}$. The organic extracts were dried over $\mathrm{MgSO}_{4}$ and concentrated in vacuo. Flash chromatography (3:1 petroleum ether: EtOAc) provided 25 as a colourless solid $(0.15 \mathrm{~g}, 81 \%)$. Two recrystallisations from hexanes/EtOAc provided a sample of the major diastereoisomer (20:1), Mp 128-130 ${ }^{\circ} \mathrm{C},{ }^{1} \mathrm{H}$ NMR $(250 \mathrm{MHz}$, $\left.\mathrm{CDCl}_{3}\right): \delta 0.98(1 \mathrm{H}, \mathrm{d}, J=6.0 \mathrm{~Hz}), 1.29(3 \mathrm{H}, \mathrm{d}, J=6.5 \mathrm{~Hz}), 1.35-1.41(1 \mathrm{H}, \mathrm{m}), 1.80(1 \mathrm{H}$, $\mathrm{d}, J=3.0 \mathrm{~Hz}), 2.45(3 \mathrm{H}, \mathrm{s}), 4.90-4.99(1 \mathrm{H}, \mathrm{m}), 7.18-7.41(15 \mathrm{H}, \mathrm{m}), 7.69(2 \mathrm{H}, \mathrm{d}, J=8.5$ $\mathrm{Hz}) ;{ }^{13} \mathrm{C} \mathrm{NMR}\left(62.9 \mathrm{MHz}, \mathrm{CDCl}_{3}\right): \delta 16.5,21.6,23.3,34.9,74.1,79.8,126.8,127.5$, 127.7, 129.3, 129.7, 134.4, 143.9, 144.3, FTIR (film, $v_{\max } \mathrm{cm}^{-1}$ ) 2922 (s), 2851 (s), 1597 (w), 1447 (w), 1364 (s), HRMS (FAB) $m / z\left(\mathrm{MH}^{+}\right.$) calcd for $\mathrm{C}_{30} \mathrm{H}_{30} \mathrm{NO}_{3} \mathrm{~S}$ 484.1946, found 484.1945 .

2(S)-(1(R)-Azidoethyl)-1-tritylaziridine 26. To a solution of $25(0.1 \mathrm{~g}, 0.21 \mathrm{mmol}, 1$ eq) in DMF (0.7 mL) was added sodium azide (67 mg, $1.03 \mathrm{mmol}, 5 \mathrm{eq})$. The solution was heated to reflux and left overnight. Upon cooling diethyl ether was added and the reaction quenched with brine. The organic extracts were washed with water, dried over $\mathrm{MgSO}_{4}$ and concentrated in vacuo. Flash chromatography (5:1 petroleum ether: EtOAc) provided 26 as a colourless solid (54 mg, 72\%). Mp 90-92 $\mathrm{C},[\alpha]^{22}-20.0$ (c 1.6, 
$\left.\mathrm{CHCl}_{3}\right),{ }^{1} \mathrm{H} \mathrm{NMR}\left(250 \mathrm{MHz}, \mathrm{CDCl}_{3}\right): \delta 1.13(1 \mathrm{H}, \mathrm{d}, J=6.0 \mathrm{~Hz}), 1.21(3 \mathrm{H}, \mathrm{d}, J=7.0 \mathrm{~Hz})$, 1.35-1.38 (1H, m), $1.94(1 \mathrm{H}, \mathrm{d}, J=3.0), 3.71-3.77(1 \mathrm{H}, \mathrm{m}), 7.21-7.34(9 \mathrm{H}, \mathrm{m}), 7.50-7.55$ $(6 \mathrm{H}, \mathrm{m}) ;{ }^{13} \mathrm{C}$ NMR $\left(62.9 \mathrm{MHz}, \mathrm{CDCl}_{3}\right): \delta 18.0,24.1,37.3,58.5,74.1,126.7,127.5$, 129.4, 144.2, FTIR (film, $v_{\max } \mathrm{cm}^{-1}$ ) 3058 (w) 2922 (m), 2854 (m), 2097 (s), 1596 (m), HRMS (EI) $m / z\left(\mathrm{MH}^{+}\right)$calcd for $\mathrm{C}_{23} \mathrm{H}_{22} \mathrm{~N}_{4} 354.1844$, found 354.1829.

2(R)-(1(S)-Phenylethyl)-1-tosylaziridine 27 To a solution of $\mathbf{2 5}(90 \mathrm{mg}, 0.19 \mathrm{mmol}, 1$ eq) and CuBr.DMS (10 mg, $0.05 \mathrm{mmol}, 0.25 \mathrm{eq})$ in THF $(1.2 \mathrm{~mL})$ was added freshly prepared phenylmagnesium bromide solution (titrated to $0.32 \mathrm{M}, 1.1 \mathrm{~mL}, 0.37 \mathrm{mmol}, 2$ eq) at $-78{ }^{\circ} \mathrm{C}$. The resulting solution was stirred at $-78{ }^{\circ} \mathrm{C}$ for 15 minutes and allowed to warm to $\mathrm{rt}$ overnight. The reaction mixture was quenched with water, poured onto brine and extracted with ethyl acetate. The combined organic extracts were dried over $\mathrm{MgSO}_{4}$ and concentrated in vacuo. Flash chromatography (10:1 petroleum ether: EtOAc) provided an impure sample of the substitiution product. To a solution of this residue (57 $\mathrm{mg}, 0.15 \mathrm{mmol}, 1 \mathrm{eq})$ in a mixture of chloroform $(1.3 \mathrm{~mL})$ and methanol $(0.4 \mathrm{~mL})$ was added TFA $(0.34 \mathrm{~mL}, 4.4 \mathrm{mmol}, 30$ eq. $)$ at $-78{ }^{\circ} \mathrm{C}$. The solution was stirred at $-78{ }^{\circ} \mathrm{C}$ for 15 minutes then, allowed to warm to $0{ }^{\circ} \mathrm{C}$ for 4 hours upon which triethylamine $(0.67$ $\mathrm{mL}, 4.79 \mathrm{mmol}, 33 \mathrm{eq}$ ) was added to the solution at $0{ }^{\circ} \mathrm{C}$. After $10 \mathrm{mins}$, a solution of tosyl chloride ( $33 \mathrm{mg}, 0.17 \mathrm{mmol}, 1.2 \mathrm{eq}$ ) in chloroform $(0.3 \mathrm{~mL})$ was added via cannula and the mixture was stirred overnight at $0{ }^{\circ} \mathrm{C} . \mathrm{NaHCO}_{3}$ solution was added and the solution was extracted with chloroform. The organic extracts were dried over $\mathrm{MgSO}_{4}$ and concentrated in vacuo and the product purified by flash chromatography to provide $\mathbf{2 7}$ as a white solid (30 mg, 54\%) Mp 59-60 ${ }^{\circ} \mathrm{C},[\alpha]^{22}{ }_{\mathrm{D}}-4.4\left(c 2.3, \mathrm{CHCl}_{3}\right),{ }^{1} \mathrm{H} \mathrm{NMR}(250 \mathrm{MHz}$, $\left.\mathrm{CDCl}_{3}\right): \delta 1.21(3 \mathrm{H}, \mathrm{d}, J=7.0 \mathrm{~Hz}), 2.18(1 \mathrm{H}, \mathrm{d}, J=4.5 \mathrm{~Hz}), 2.43-2.50(4 \mathrm{H}, \mathrm{m}), 2.64(1 \mathrm{H}$, $\mathrm{d}, J=7.0 \mathrm{~Hz}), 2.85-2.90(1 \mathrm{H}, \mathrm{m}), 7.15-7.39(7 \mathrm{H}, \mathrm{m}), 7.86(2 \mathrm{H}, \mathrm{d}, J=8.5 \mathrm{~Hz}) ;{ }^{13} \mathrm{C} \mathrm{NMR}$ (62.9 MHz, $\left.\mathrm{CDCl}_{3}\right): \delta 18.9,21.6,33.3,41.8,45.6,126.9,127.1,128.1,128.6,129.6$, 135.0, 142.3, 144.5, FTIR (film, $v_{\max } \mathrm{cm}^{-1}$ ) 3029 (w), 2986 (w), 1698 (m), 1597 (m), 1494 (m), HRMS (TOF ESI+) $\mathrm{m} / z\left(\mathrm{MNa}^{+}\right)$calcd for $\mathrm{C}_{17} \mathrm{H}_{19} \mathrm{NO}_{2} \mathrm{NaS} 324.1034$, found 324.1024 . 

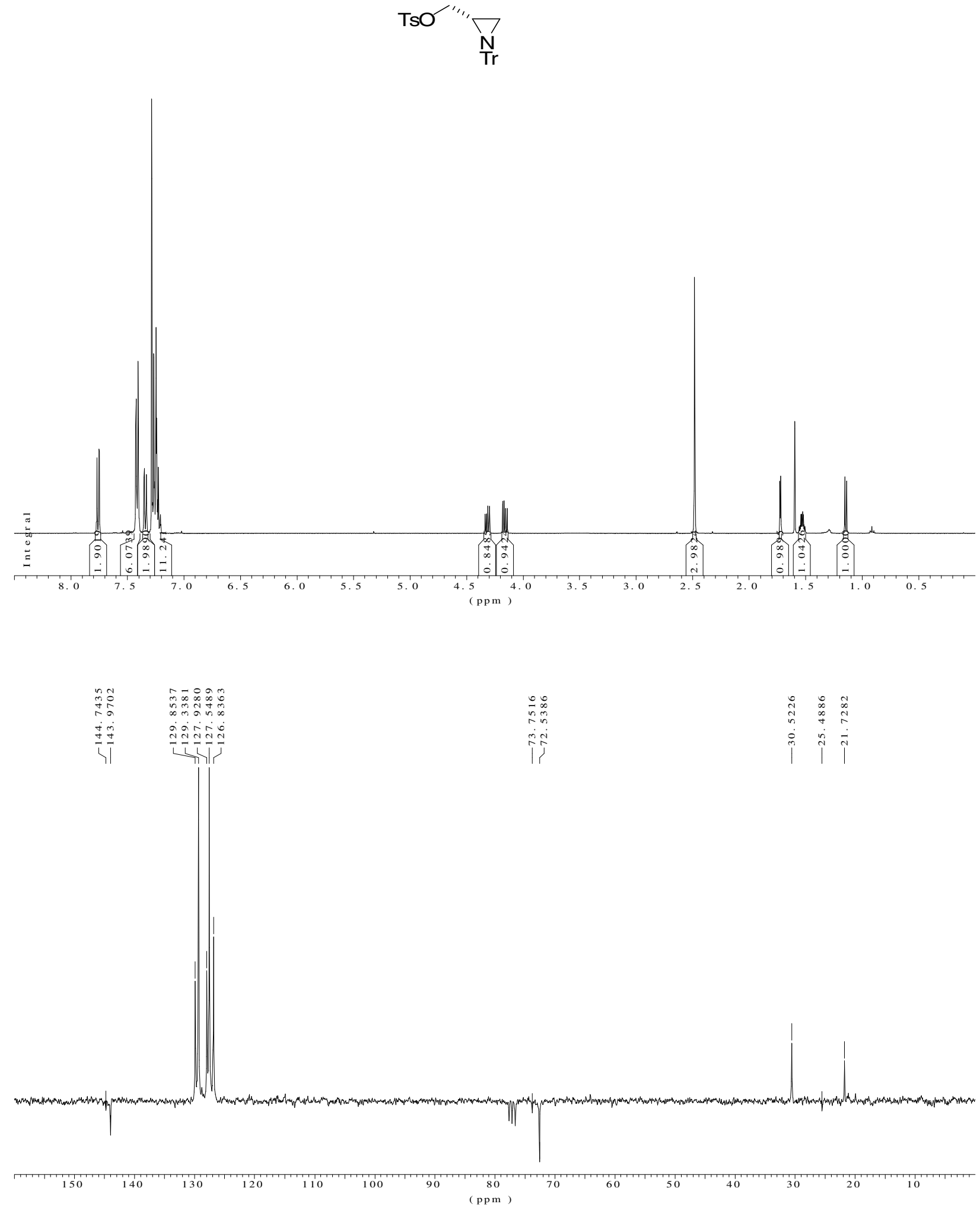
10

$$
\mathrm{Bu}^{\prime \prime \prime \prime \prime} \underset{\mathrm{Tr}}{\mathrm{N}}
$$
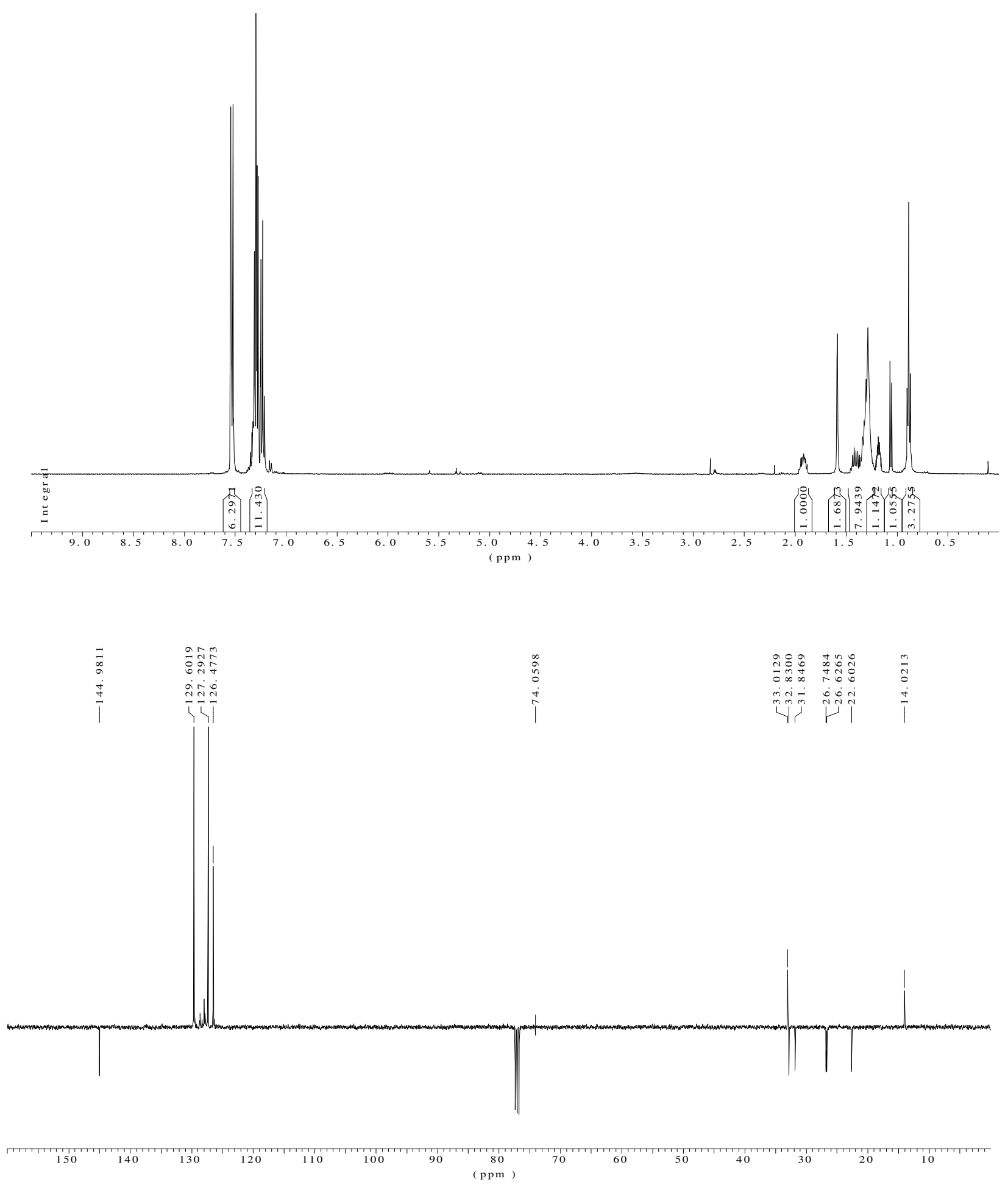


\section{1}

$$
\left(r_{2}^{\prime \prime \prime \prime}{ }^{\mathrm{N}}\right.
$$
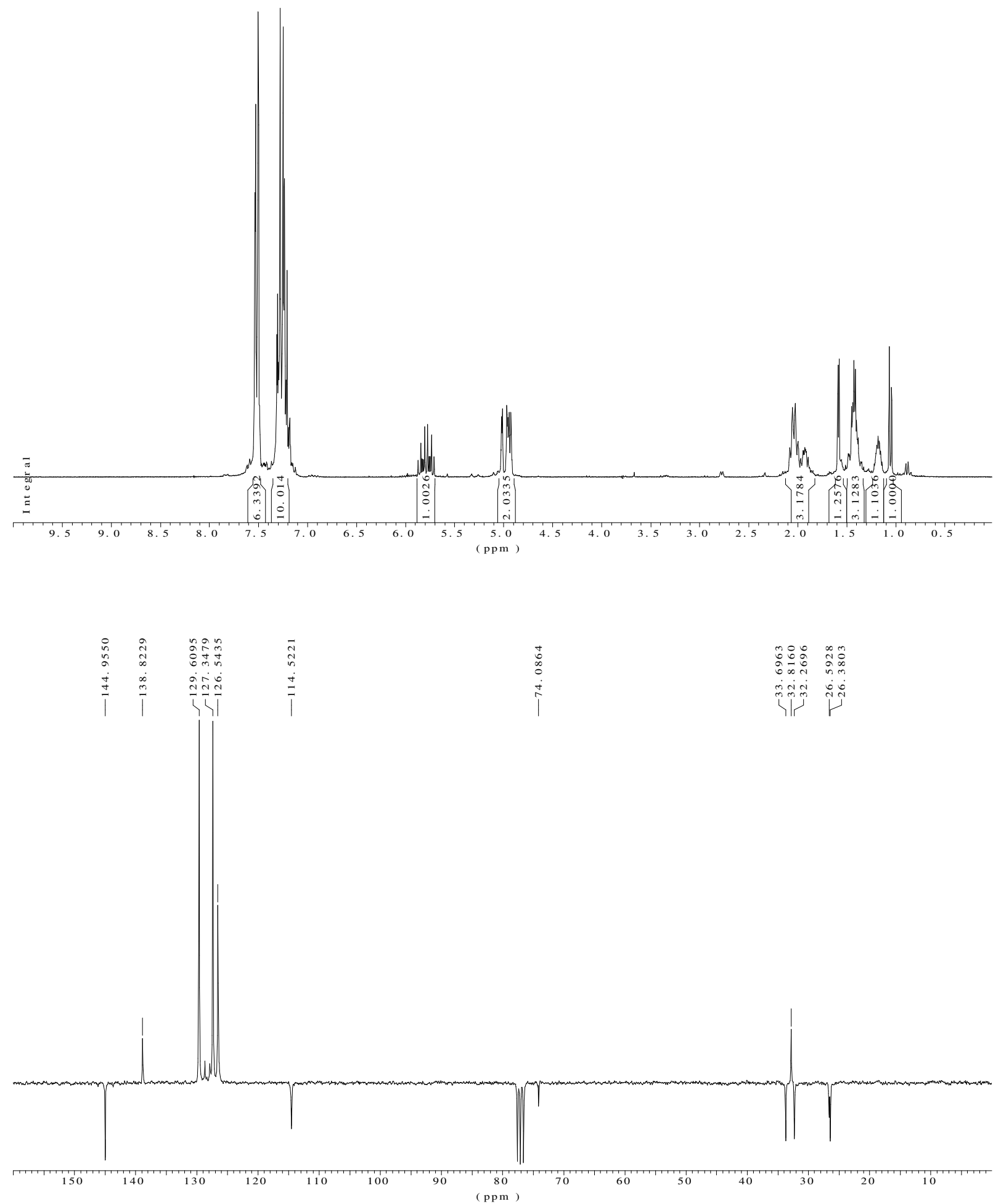
12

${ }^{\prime \prime \prime} \stackrel{\mathrm{N}}{\mathrm{Nr}}$
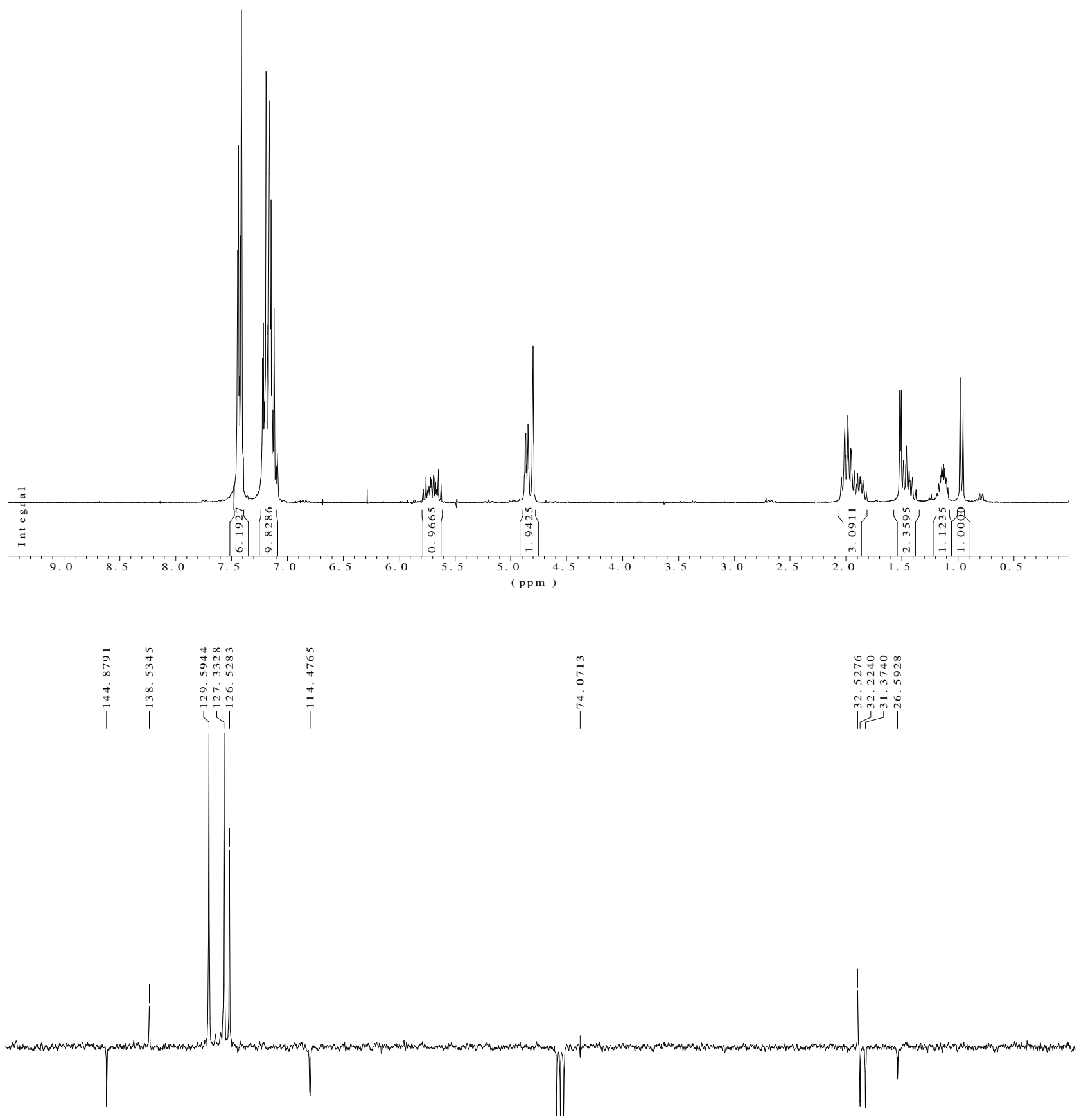
13

$\mathrm{Ph}^{\prime \prime \prime} \stackrel{\mathrm{Tr}}{\mathrm{N}}$
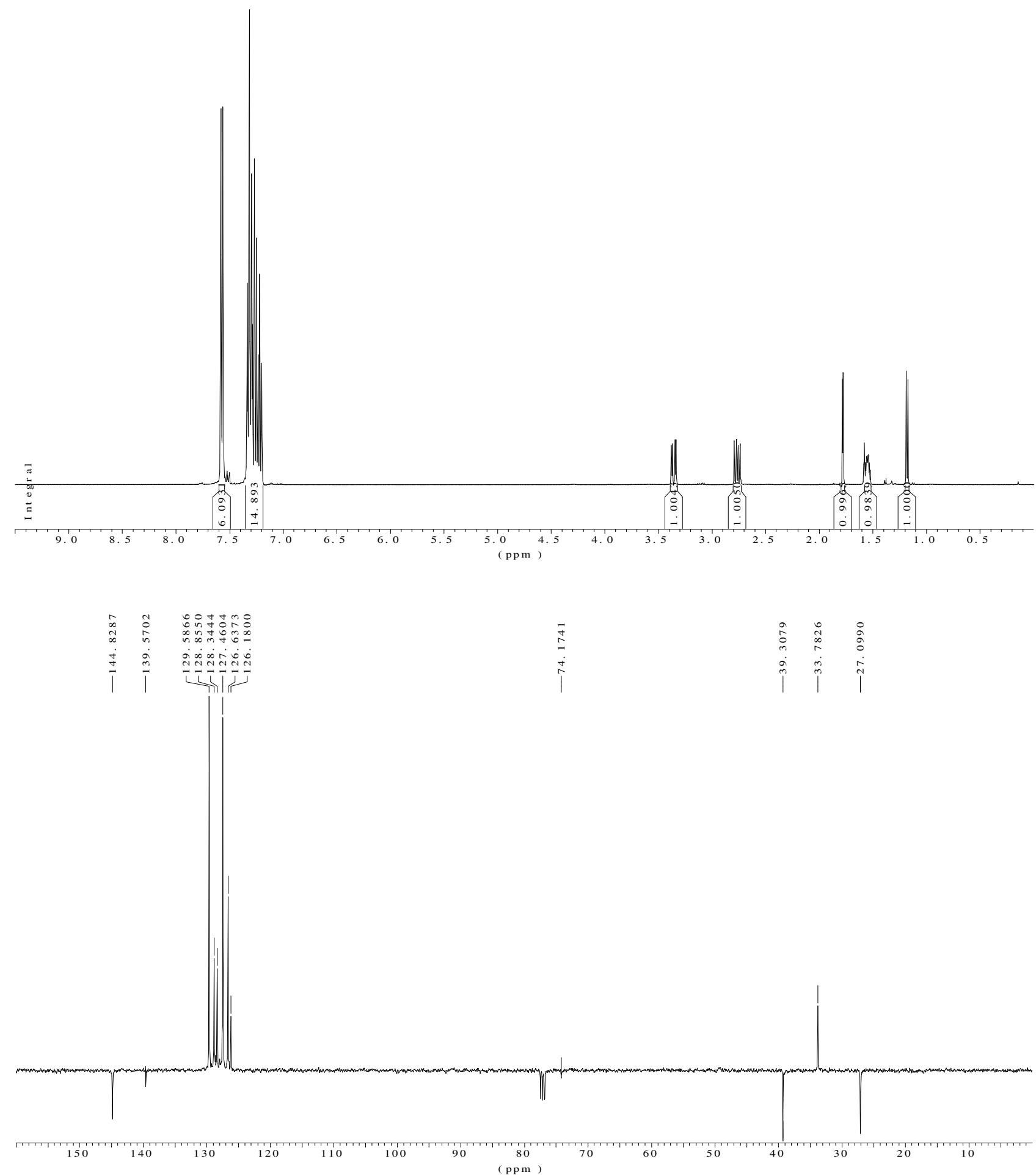


\section{4}
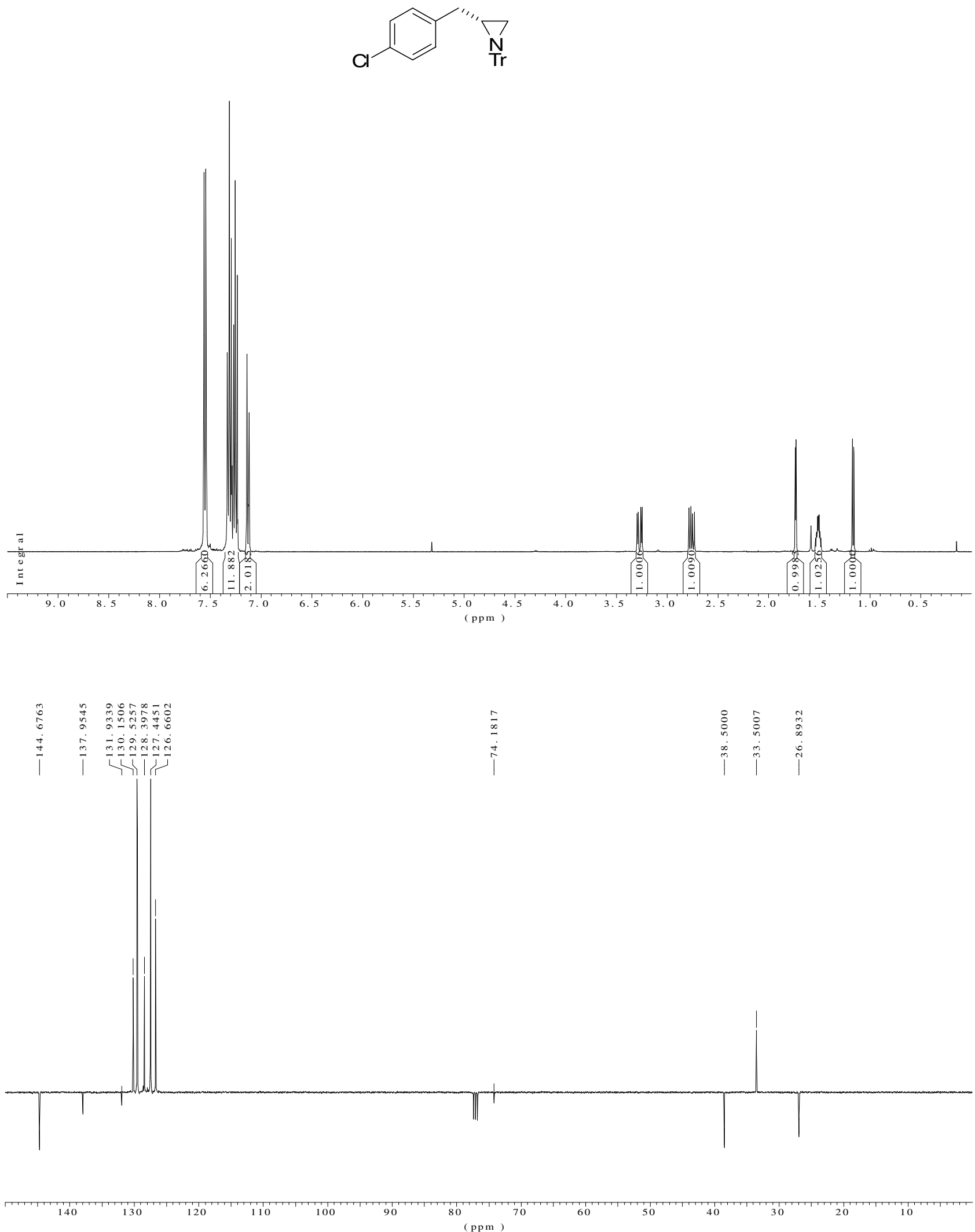
15
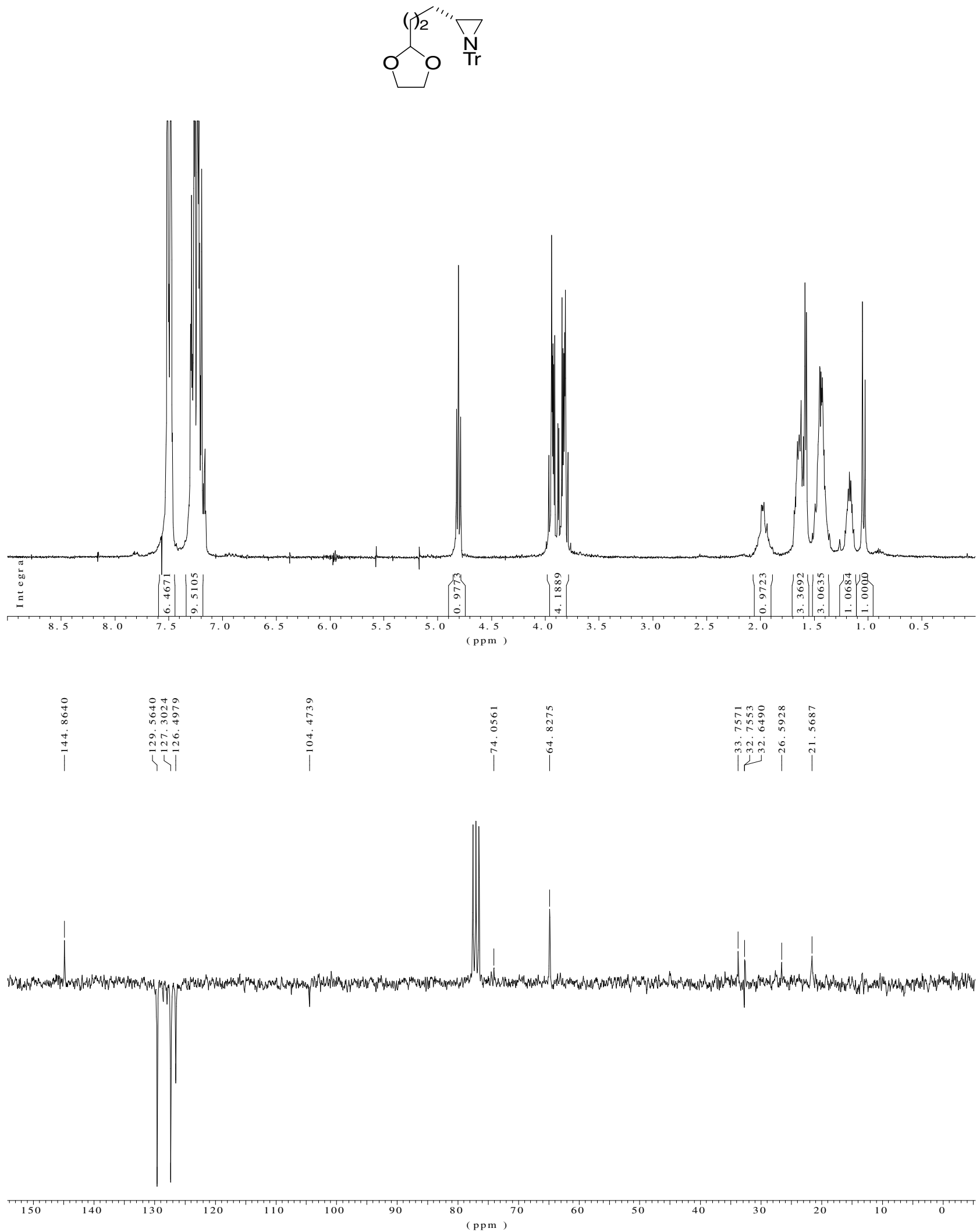
16

$$
\mathrm{Me}_{3} \mathrm{Si} \stackrel{\mathrm{N}}{\mathrm{Tr}}
$$
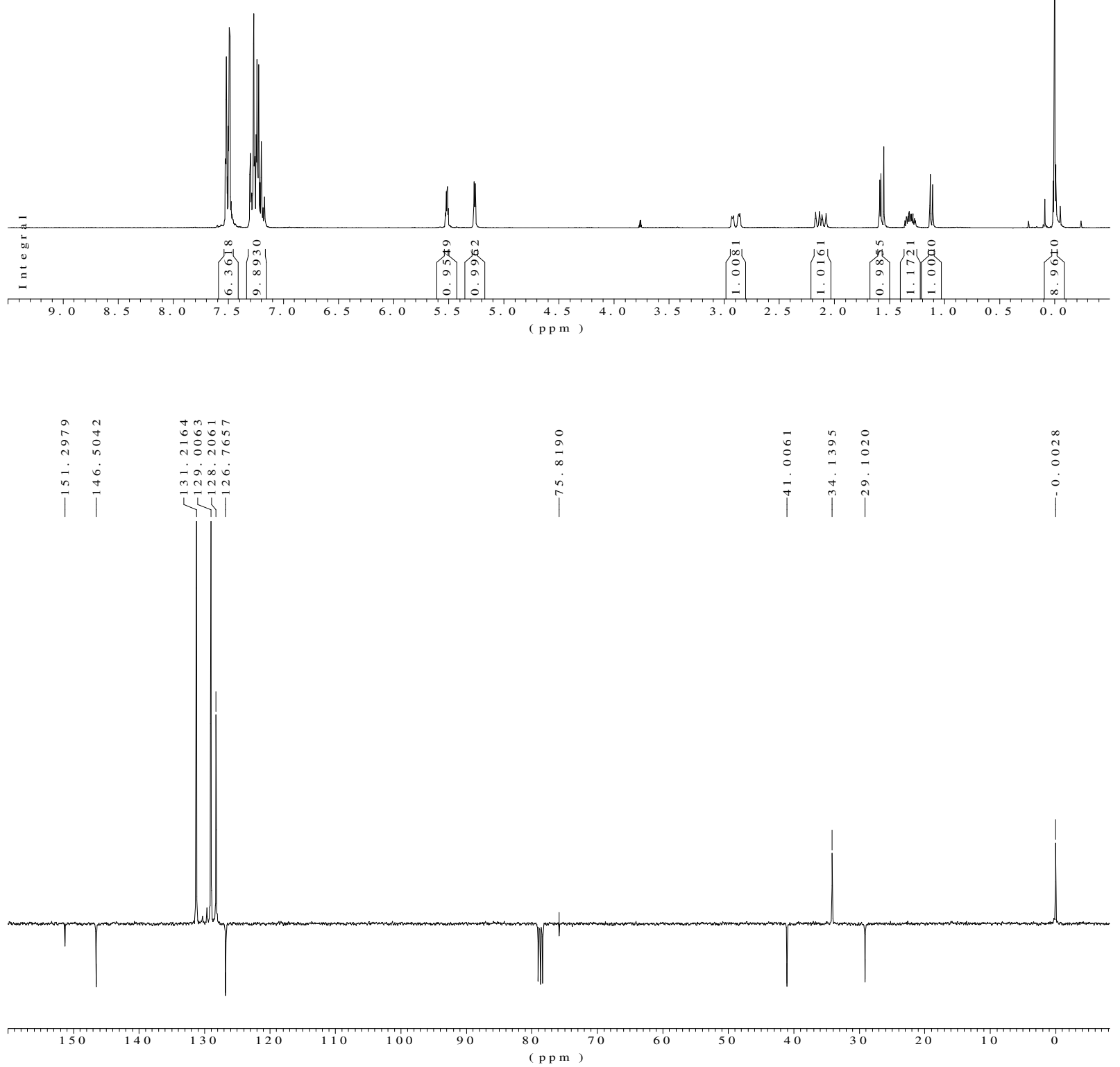
17

$$
\mathrm{N}_{3}{ }^{\prime \prime \prime} \stackrel{\mathrm{Tr}}{\mathrm{N}}
$$
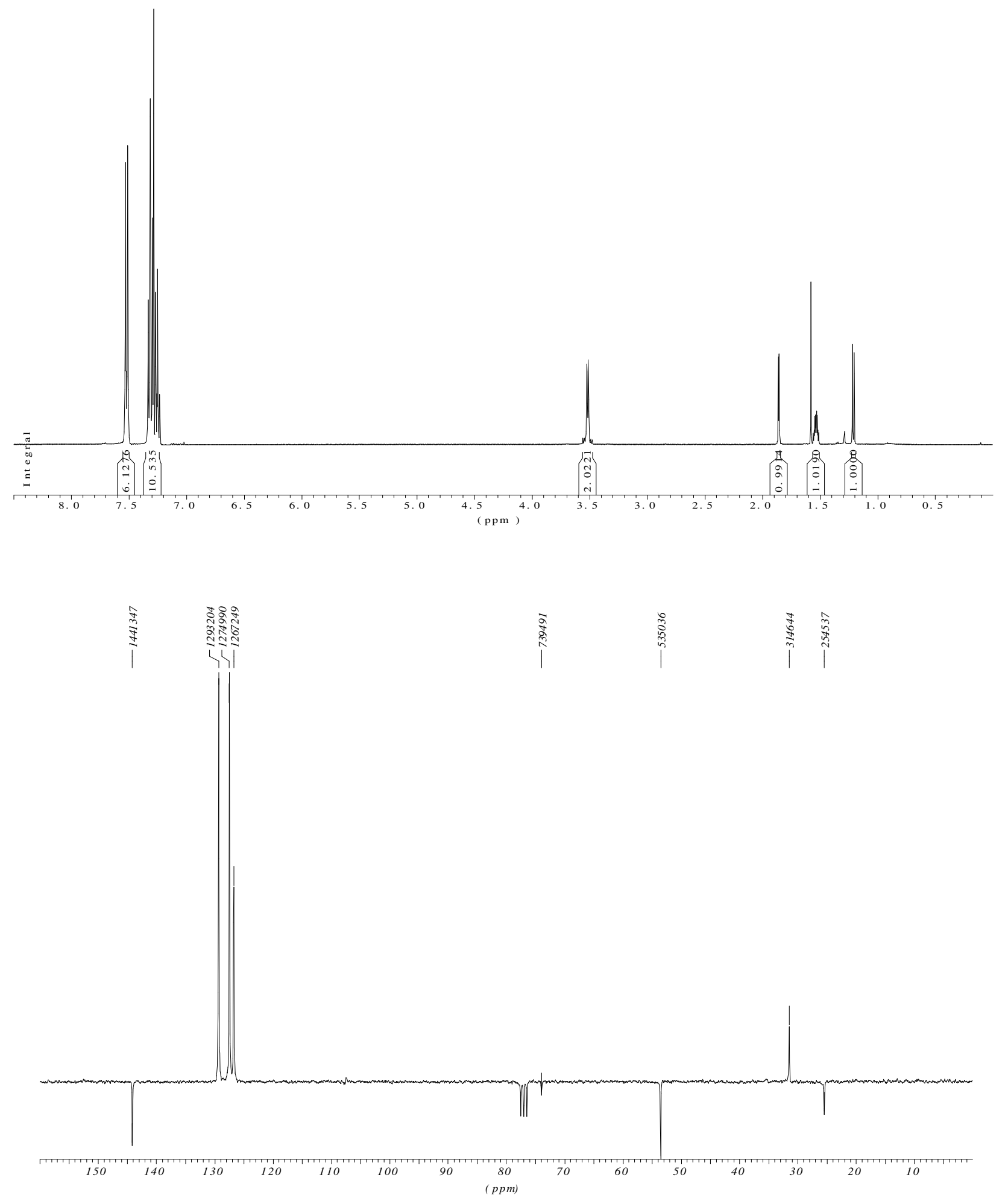
$B n$,

Ts
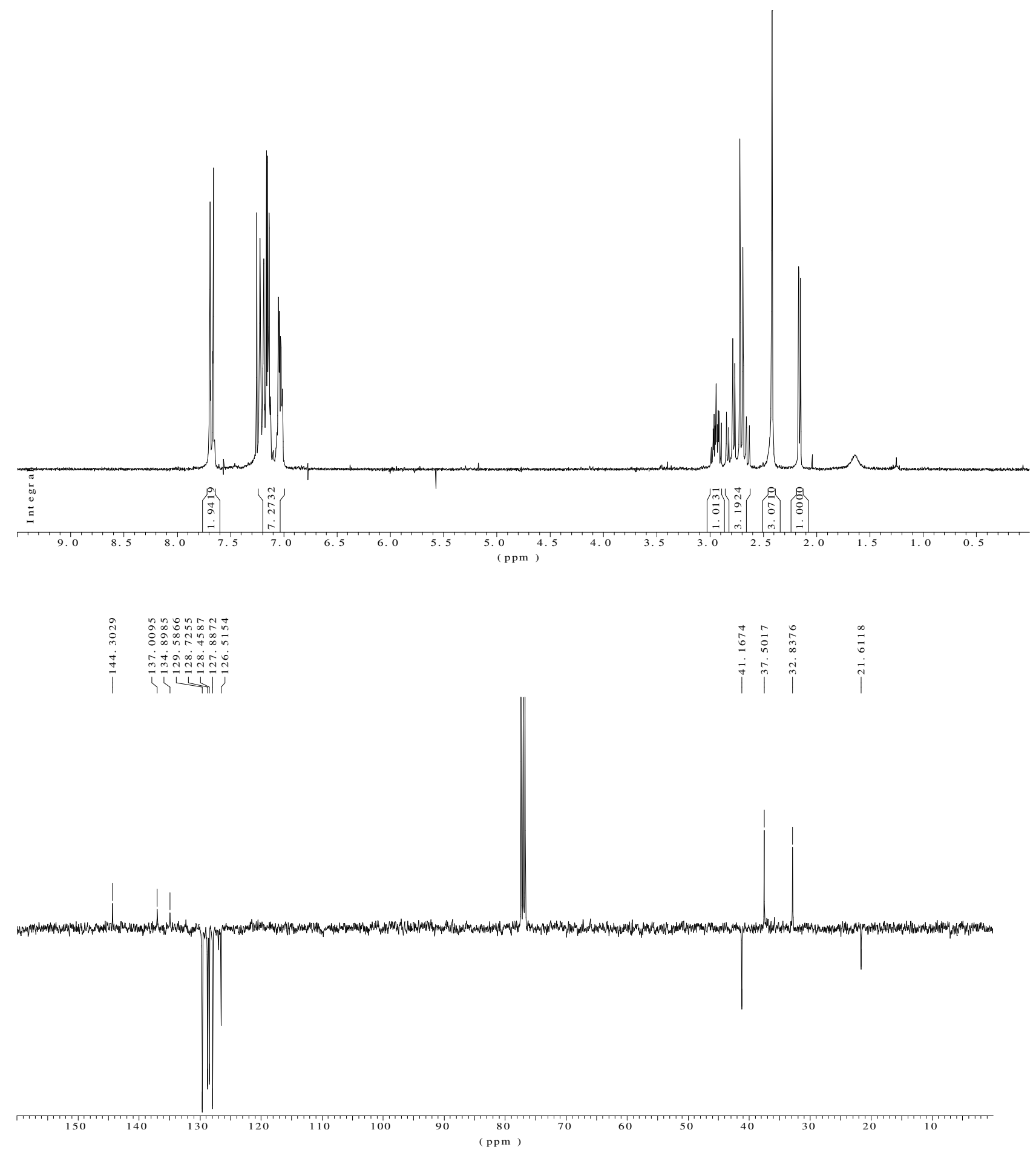
Bn, N PMBS

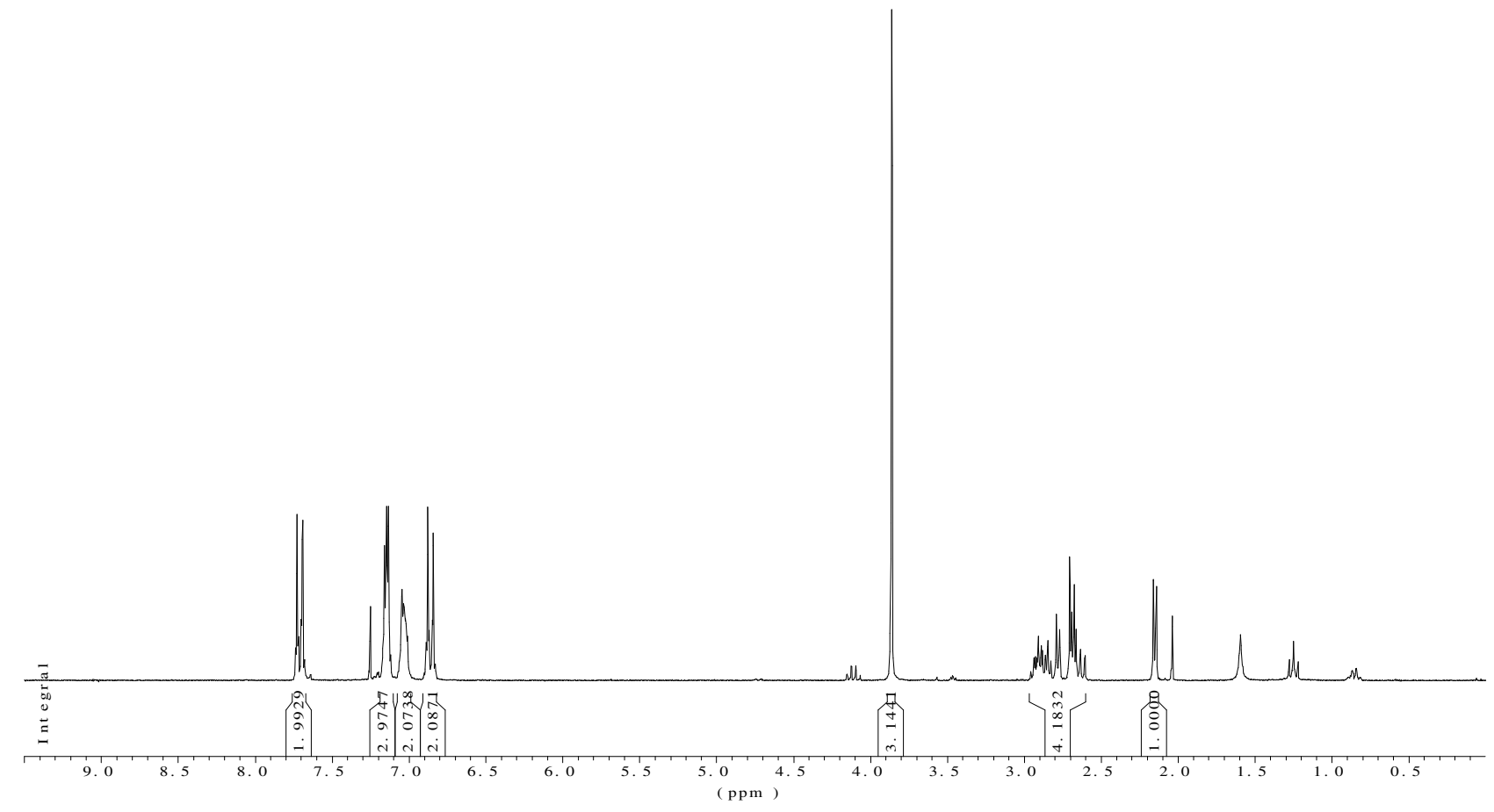

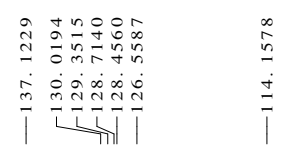

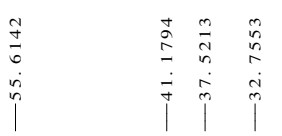

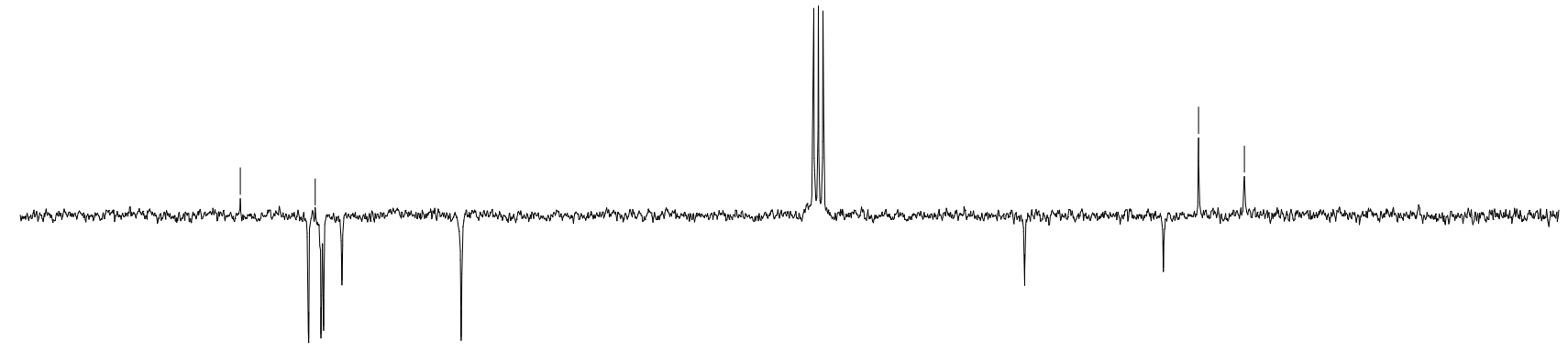

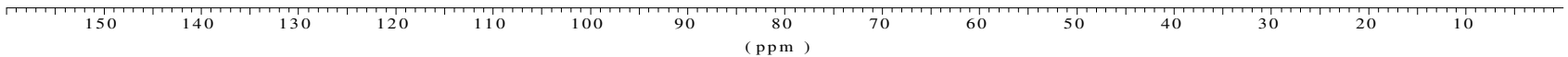


22

$\mathrm{Bn}$

$$
\text { "N }
$$
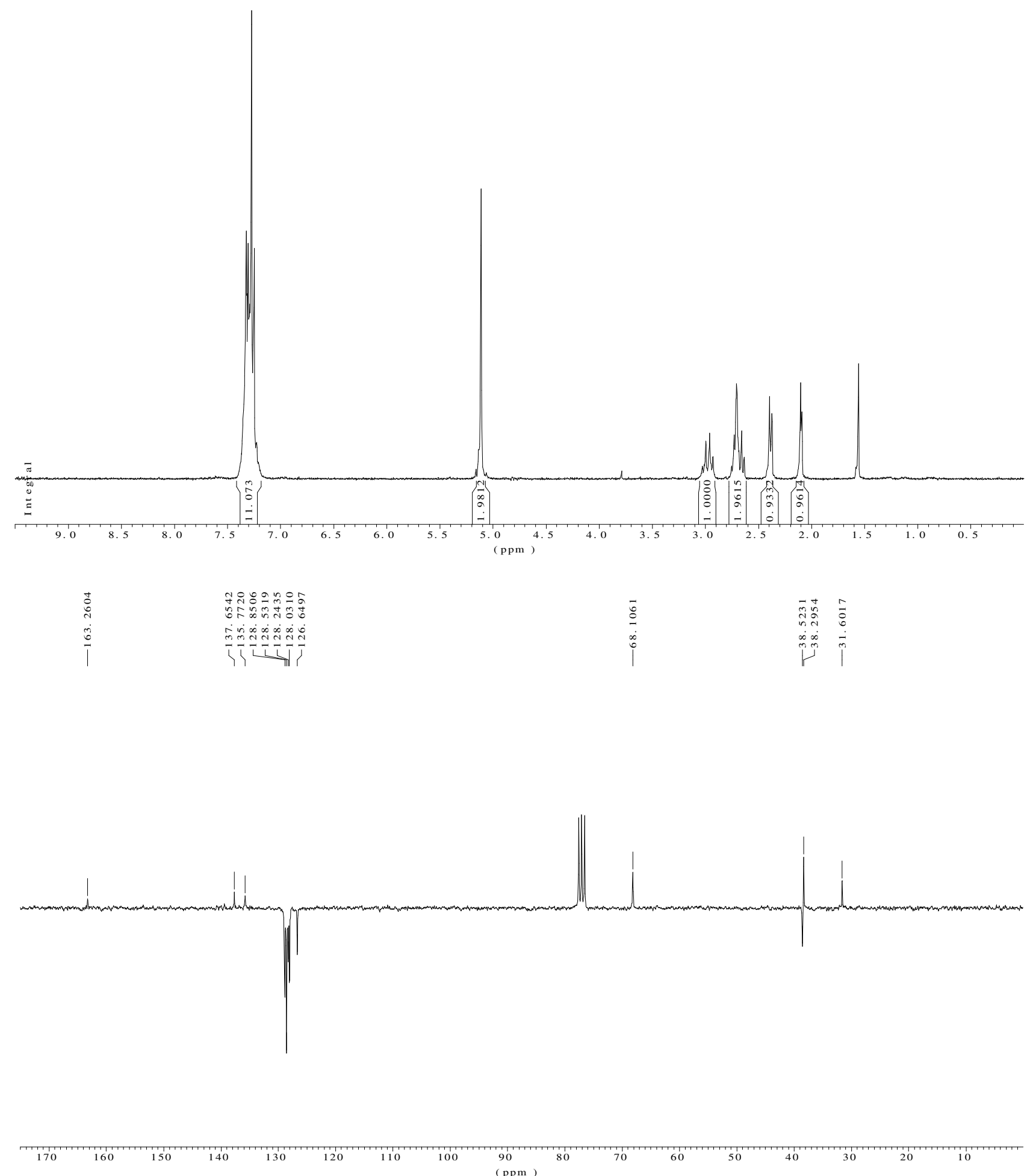
Bn,

$\stackrel{N}{\text { Dpp }}$

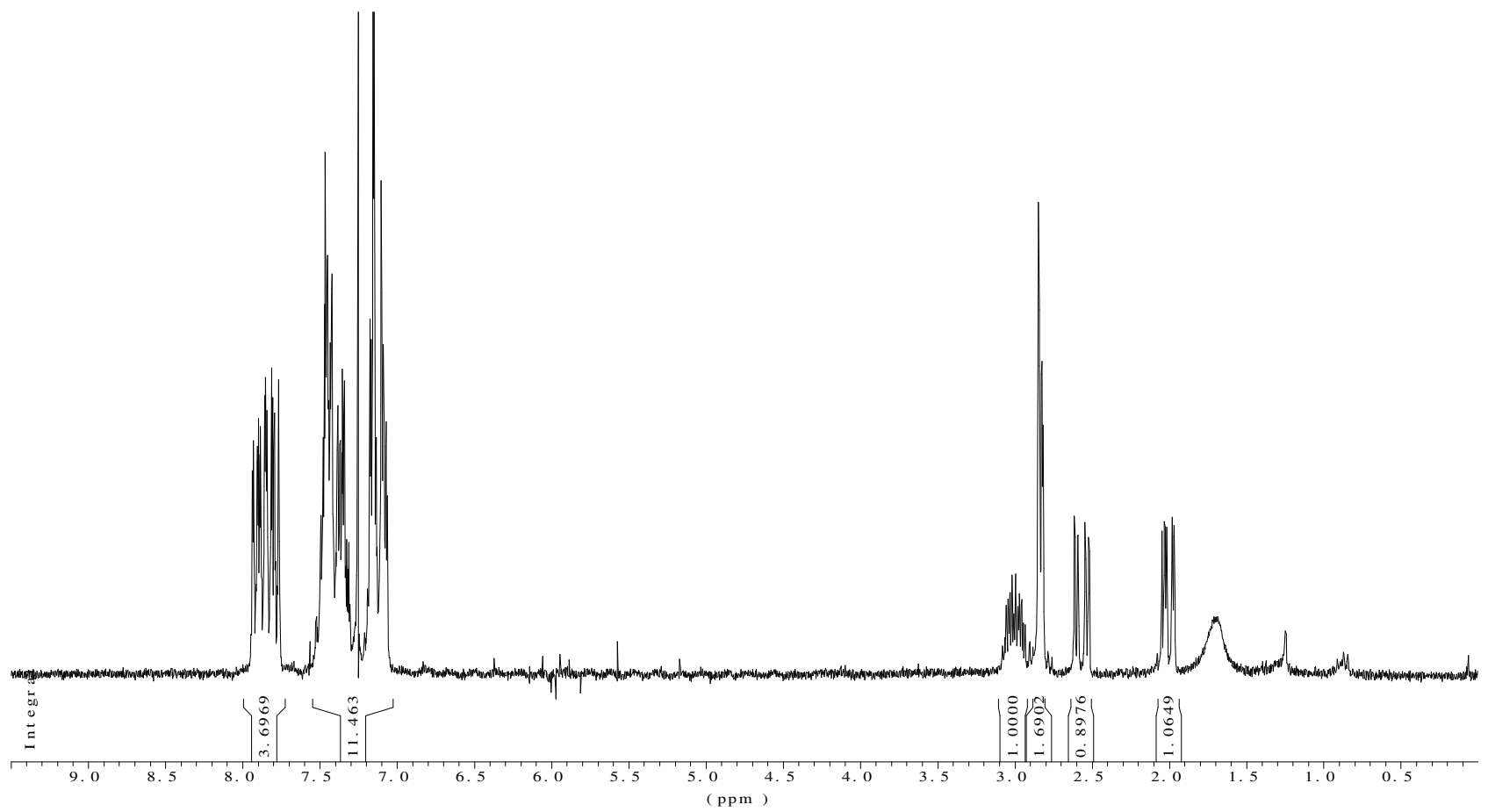


24
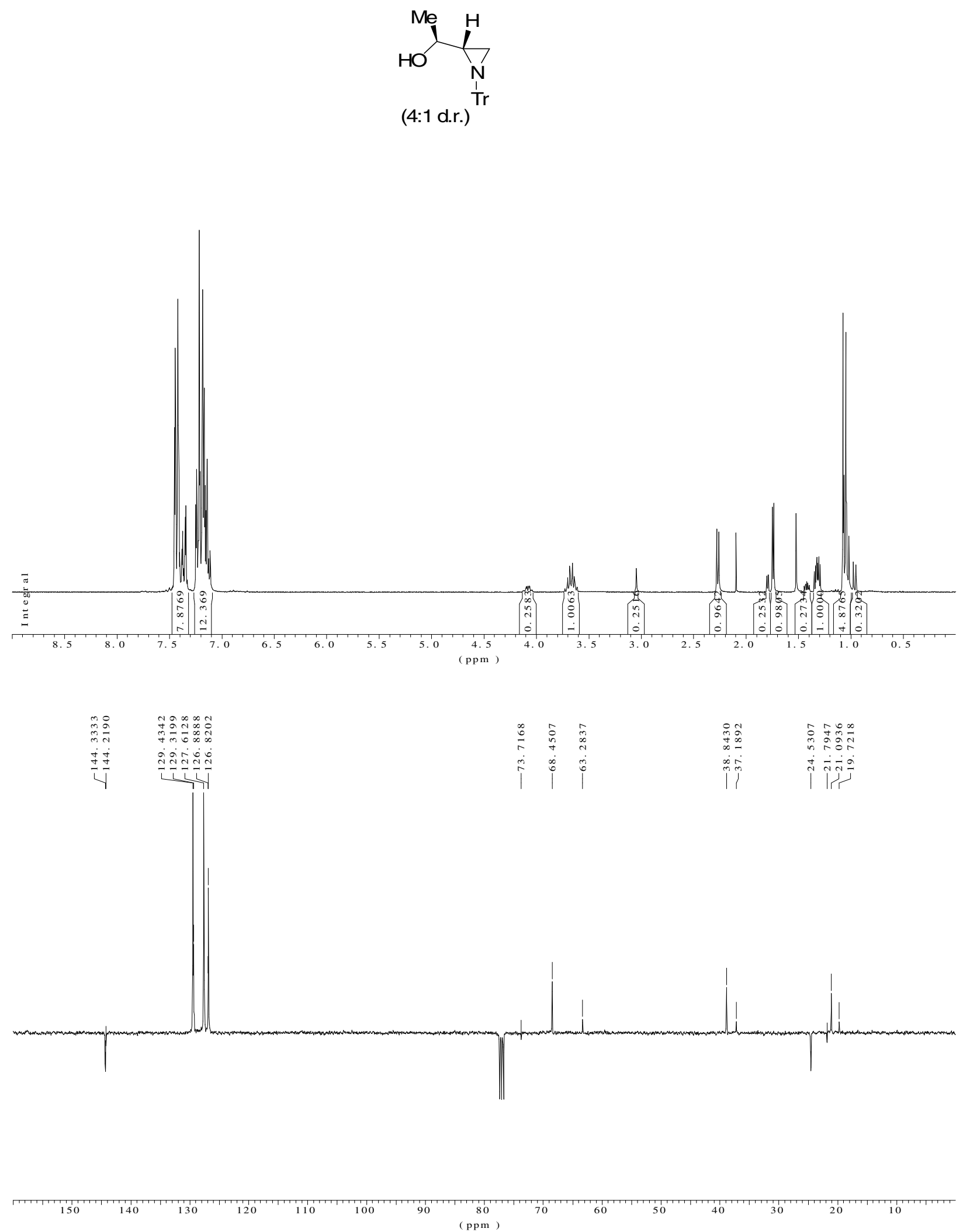

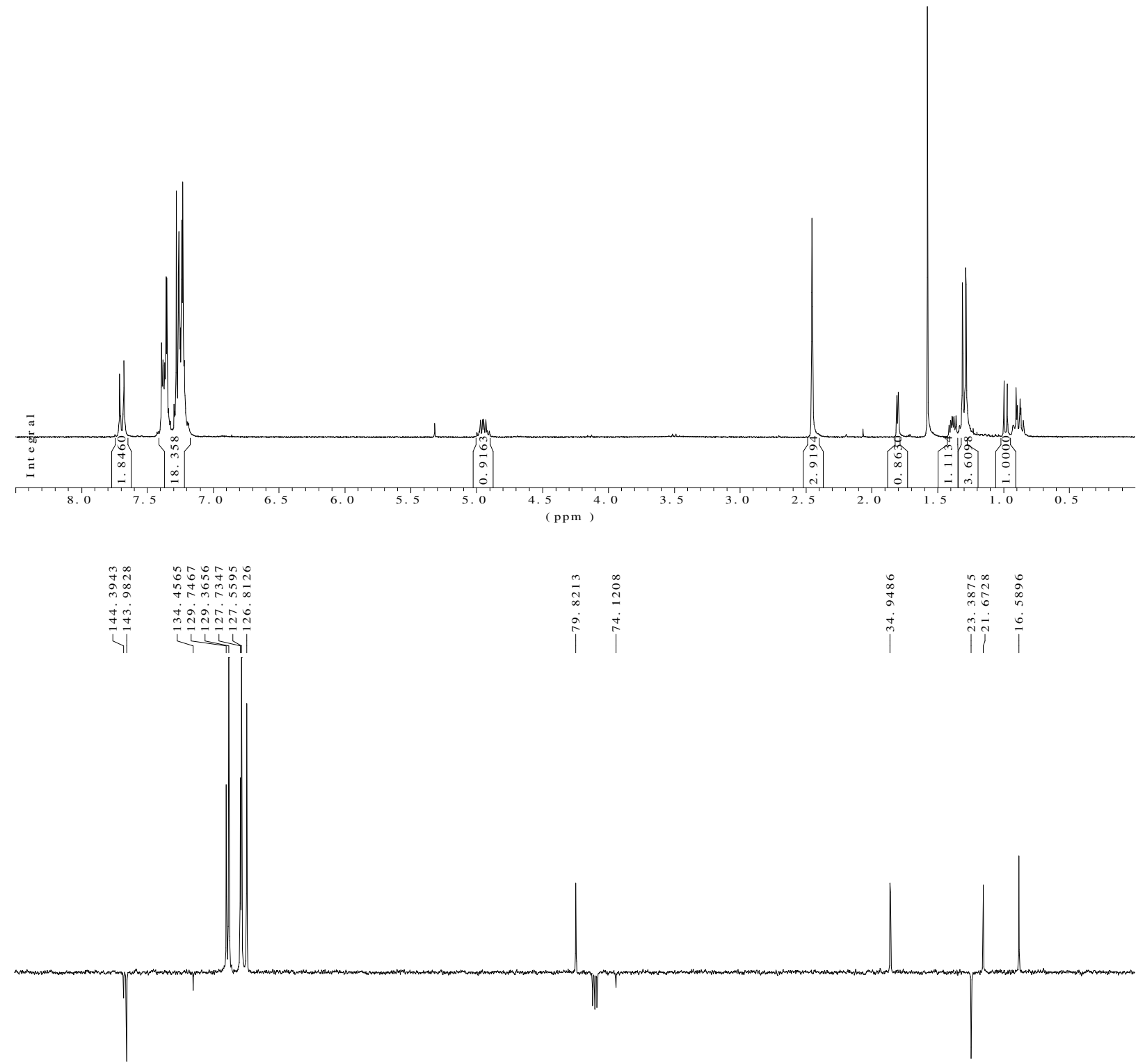

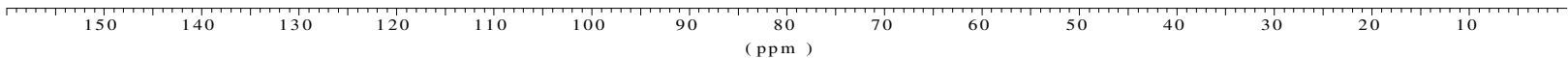




$$
\underbrace{\mathrm{Ne}}_{\substack{1 \\ \mathrm{Tr}}}
$$
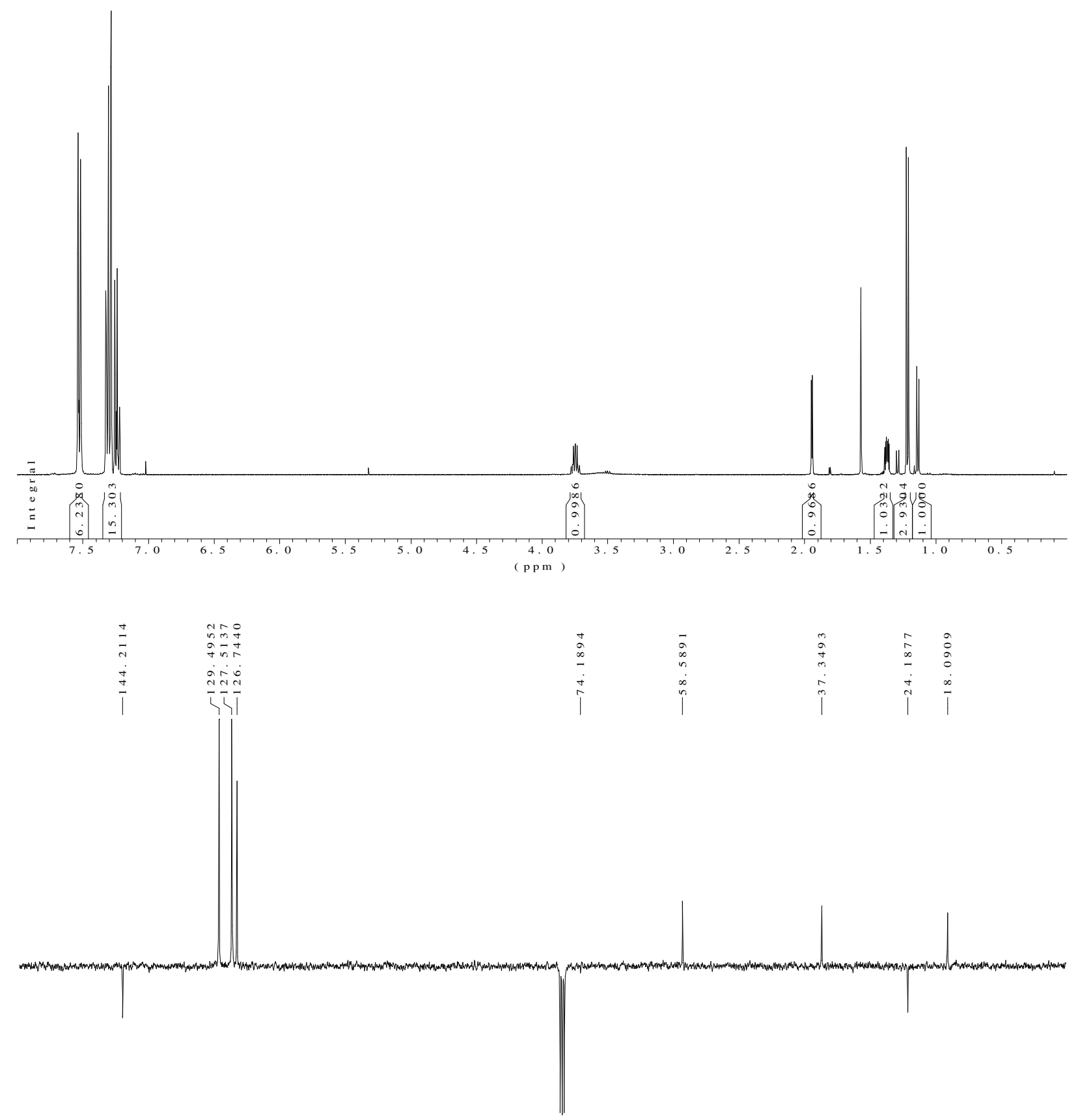

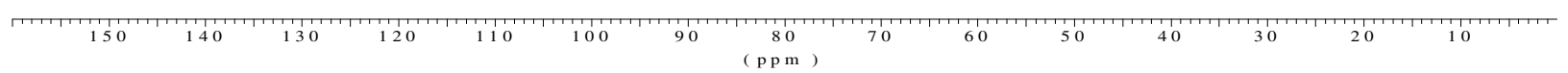



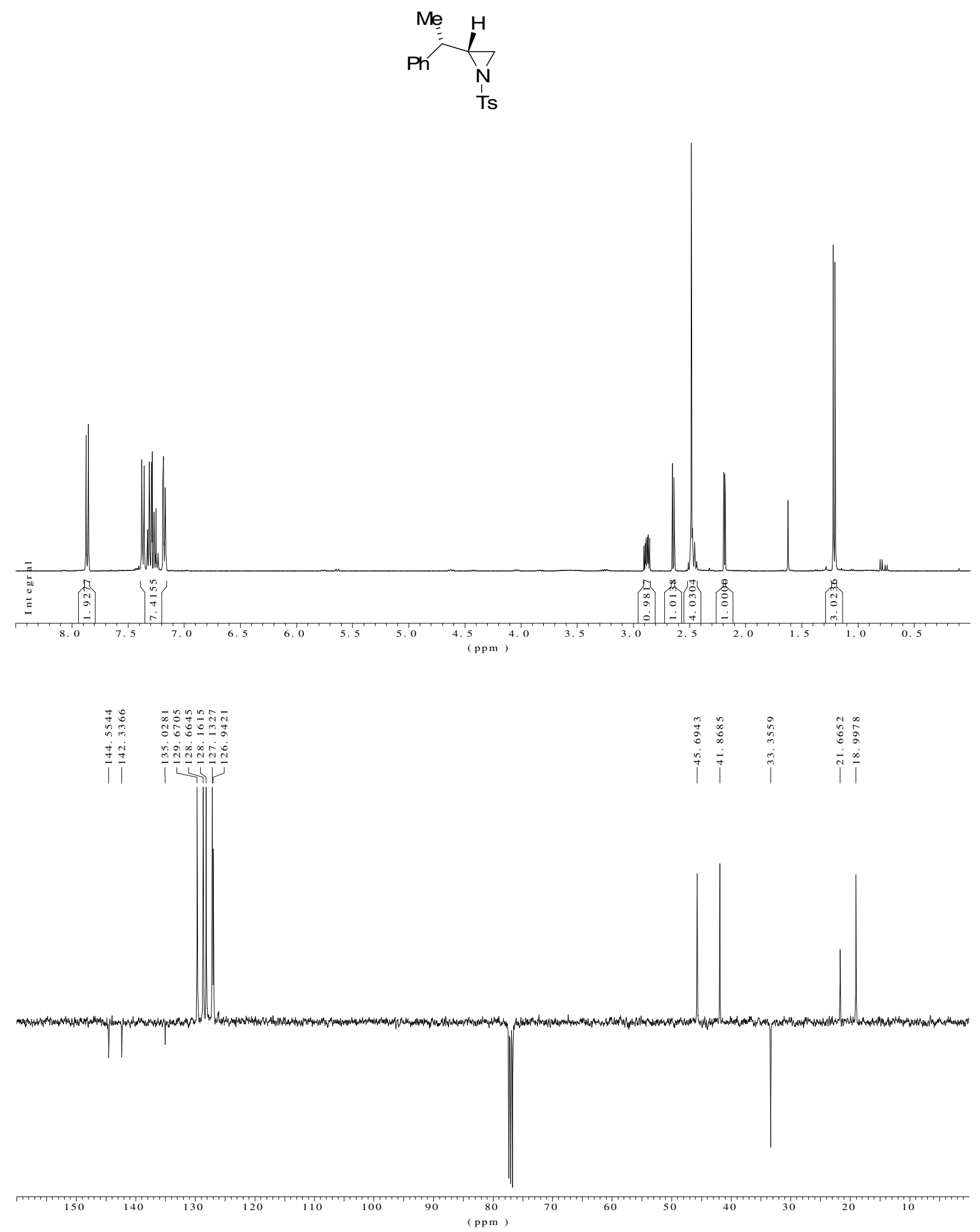
$11 / 07 / 2007 \quad 11: 40$

Created : 11/07/2007 11:26:53

Project : WORK4

Sample ID : CJ.tsaziridine

Calibration : CJ.tsaziridine

Method

Description

Created

Column

Mobile Phase

Flow Rate

Note

Detector 1

Subtraction chromatogram : (None)
Chromatogram CJ.tsaziridine

Page 1 of 1

By

Report Style : Cull

$\begin{array}{ll}\text { Report Style } & \text { Full } \\ \text { Sample } & \text { CJ.tsaziridine }\end{array}$

Chromatogram : DATAICJ.tsaziridine

By

: Clarity

Modified $\quad: 11 / 07 / 2007 \quad 11: 37$

Detection : UV@254nm

Temperature : RT

Pressure
External Start : Start Only, Down

Range 1 : Bipolar, $10000 \mathrm{mV}, 10$ Samp. per Sec.

Matching : No Change

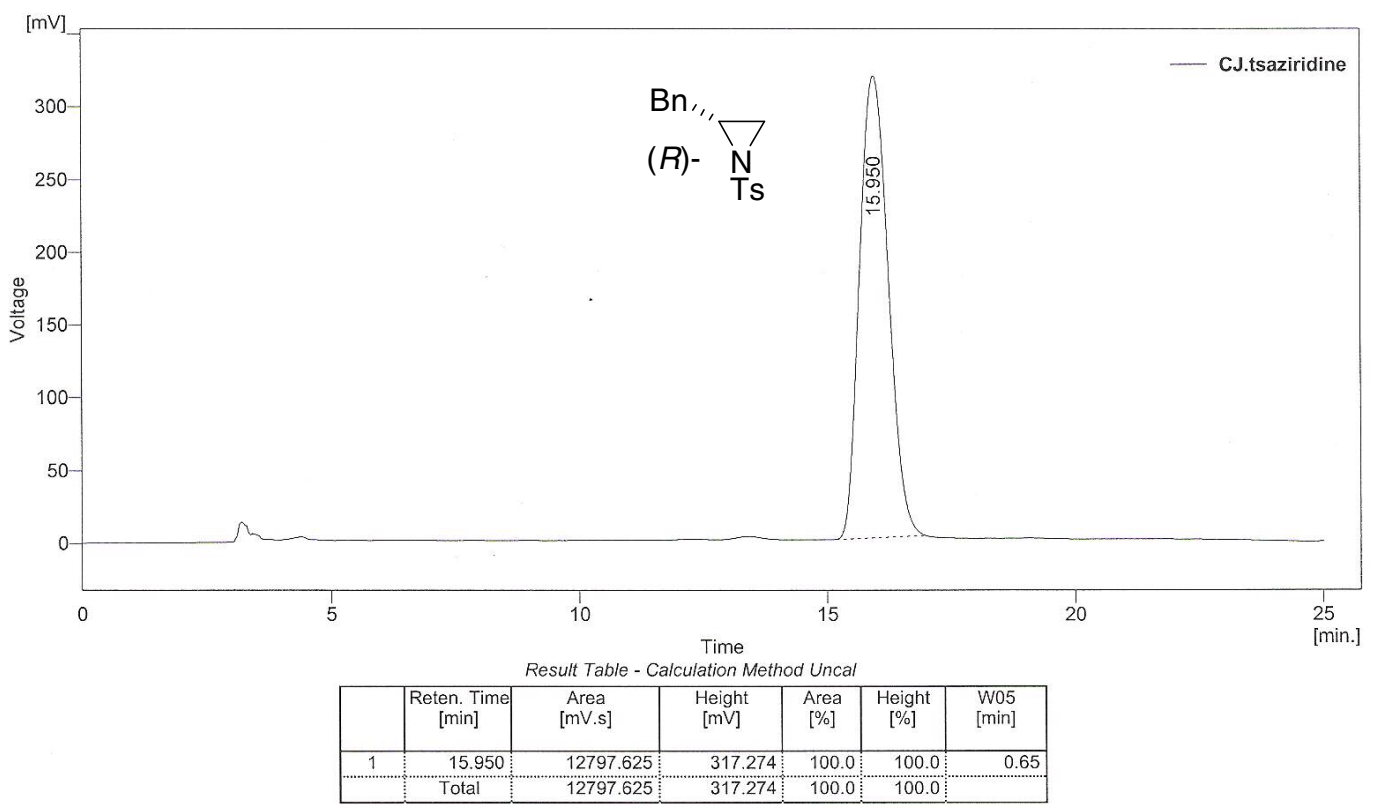




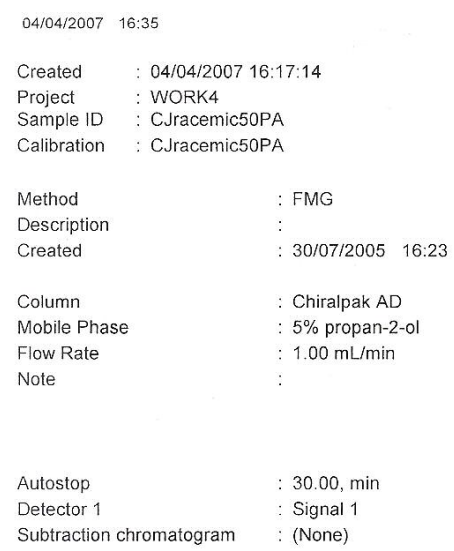

Chromatogram CJracemic50PA

: Clarity

$\begin{array}{ll}\text { Seport Style } & \text { Full } \\ \text { Sample } & \text { (1R)-(2S), B-Me 0.5\% }\end{array}$

Chromatogram : DATAICJracemic50PA

By

: Clarity

Modified $\quad:$ 04/04/2007 $\quad 16: 17$

Detection : UV@254 nm

Temperature : RT

Pressure
External Start : Start - Restart, Down

Range 1 : Bipolar, $1250 \mathrm{mV}, 10$ Samp. per Sec Matching : No Change

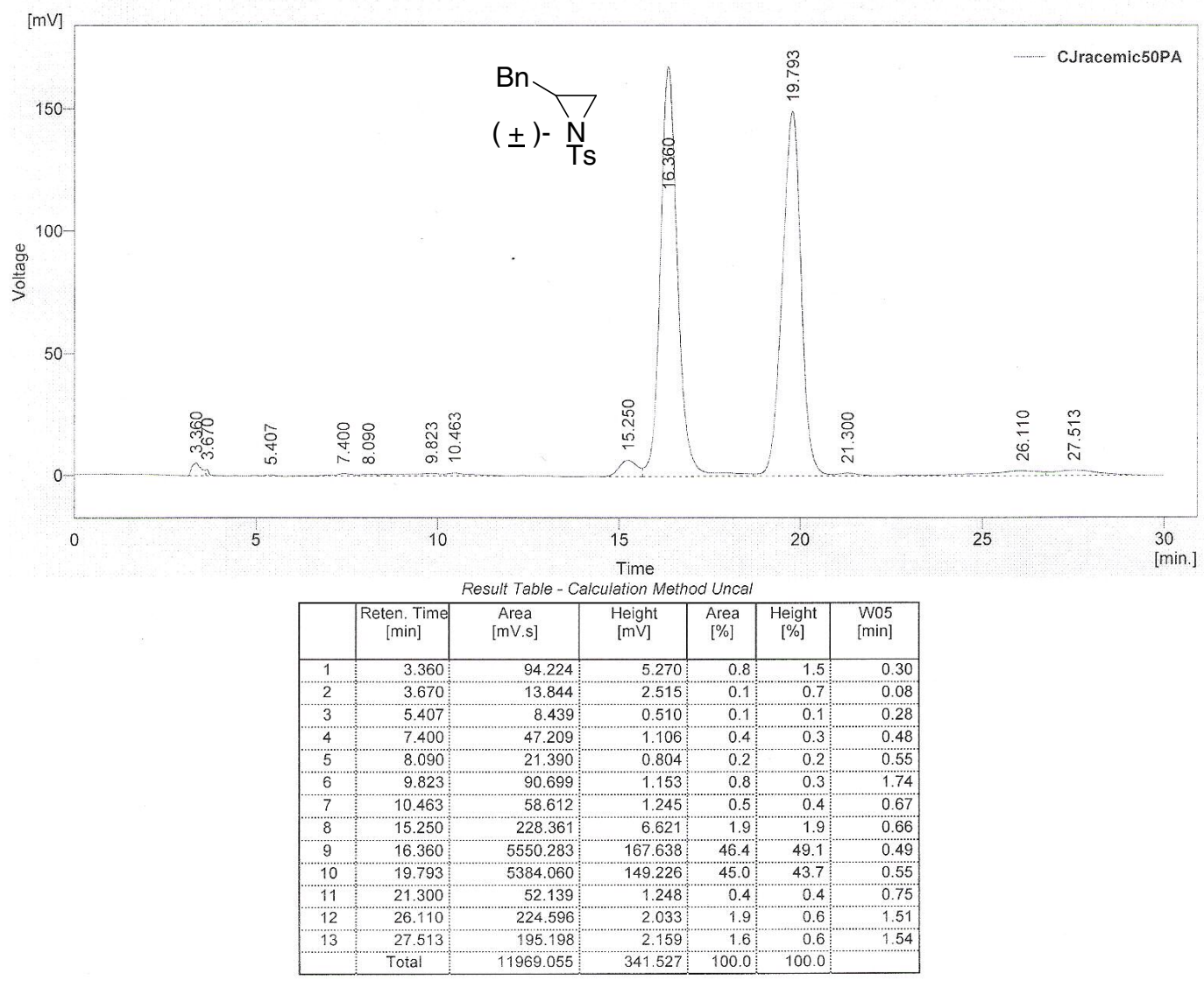




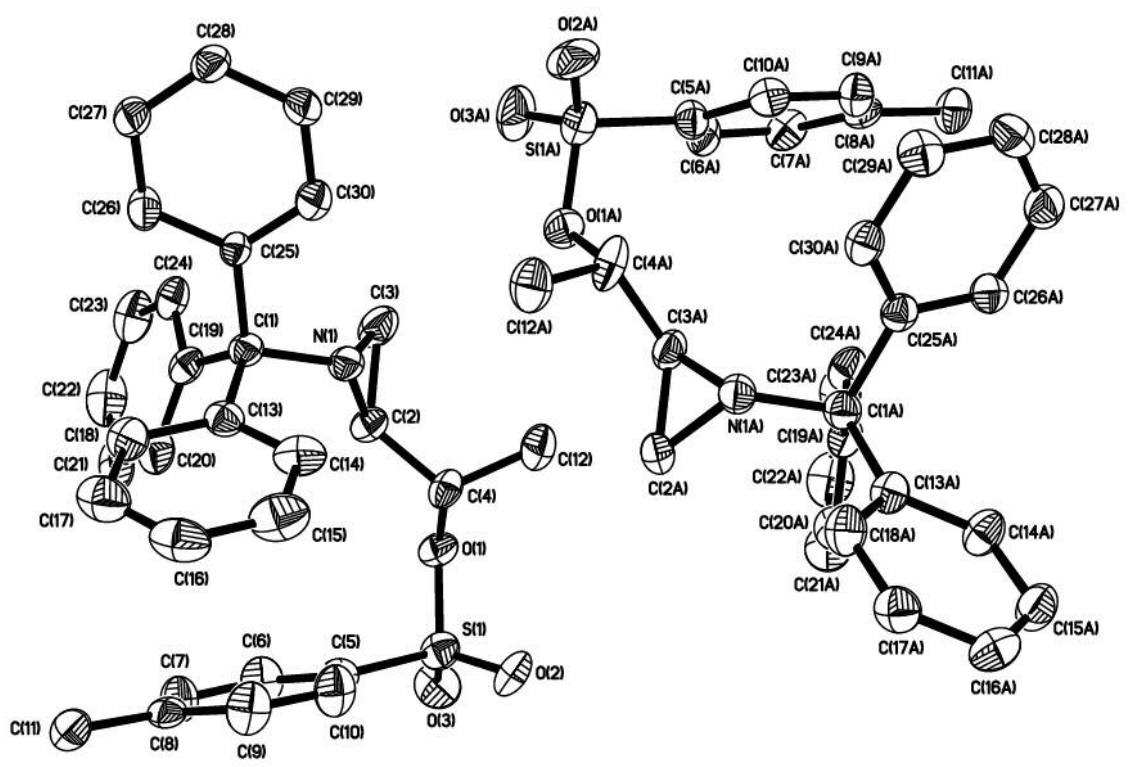

ORTEP of compound $\mathbf{2 5}$ 
Table 1. Crystal data and structure refinement for $\mathbf{2 5}$.

Identification code

Empirical formula

Formula weight

Temperature

Wavelength

Crystal system

Space group

Unit cell dimensions

Volume

$\mathrm{Z}$

Density (calculated)

Absorption coefficient

$\mathrm{F}(000)$

Crystal size

Theta range for data collection

Index ranges

Reflections collected

Independent reflections

Completeness to theta $=25.00^{\circ}$

Absorption correction

Max. and min. transmission

Refinement method

Data / restraints / parameters

Goodness-of-fit on $\mathrm{F}^{2}$

Final $\mathrm{R}$ indices $[\mathrm{I}>2 \operatorname{sigma}(\mathrm{I})]$

$\mathrm{R}$ indices (all data)

Absolute structure parameter

Largest diff. peak and hole ohj $132 \mathrm{~m}$

C30 H29 N O3 S

483.60

150(2) K

$0.71073 \AA$

Monoclinic

$\mathrm{P} 2_{1}$

$\mathrm{a}=8.947(2) \AA$

$\alpha=90^{\circ}$.

$\mathrm{b}=19.636(4) \AA$

$\beta=98.839(4)^{\circ}$.

$\mathrm{c}=14.563(3) \AA$

$\gamma=90^{\circ}$.
4

$1.271 \mathrm{Mg} / \mathrm{m}^{3}$

$0.160 \mathrm{~mm}^{-1}$

1024

$0.37 \times 0.21 \times 0.11 \mathrm{~mm}^{3}$

1.42 to $25.00^{\circ}$.

$-10<=\mathrm{h}<=10,-23<=\mathrm{k}<=23,-17<=\mathrm{l}<=17$

24258

$8921[\mathrm{R}(\mathrm{int})=0.0905]$

$100.0 \%$

Semi-empirical from equivalents

0.9826 and 0.9431

Full-matrix least-squares on $\mathrm{F}^{2}$

$8921 / 1 / 635$

0.947

$\mathrm{R} 1=0.0587, \mathrm{wR} 2=0.1307$

$\mathrm{R} 1=0.1027, \mathrm{wR} 2=0.1546$

$-0.01(9)$

0.431 and -0.353 e. $\AA^{-3}$ 
Table 2. Atomic coordinates ( x 10 $0^{4}$ ) and equivalent isotropic displacement parameters $\left(\AA^{2} \times 10^{3}\right)$ for $\mathbf{2 5}$. $U(\mathrm{eq})$ is defined as one third of the trace of the orthogonalized $\mathrm{U}^{\mathrm{ij}}$ tensor.

\begin{tabular}{|c|c|c|c|c|}
\hline & $\mathrm{x}$ & $\mathrm{y}$ & $\mathrm{z}$ & $\mathrm{U}(\mathrm{eq})$ \\
\hline $\mathrm{S}(1)$ & $10289(1)$ & 7103(1) & $7596(1)$ & $34(1)$ \\
\hline $\mathrm{N}(1)$ & $8299(4)$ & $5201(2)$ & $9148(2)$ & $29(1)$ \\
\hline $\mathrm{O}(1)$ & $10395(3)$ & $6309(2)$ & $7726(2)$ & $33(1)$ \\
\hline $\mathrm{O}(2)$ & 8982(4) & $7270(2)$ & 6933(2) & $40(1)$ \\
\hline $\mathrm{O}(3)$ & 11758(4) & $7298(2)$ & $7411(2)$ & $49(1)$ \\
\hline $\mathrm{C}(1)$ & $8329(5)$ & $5234(2)$ & 10188(3) & $26(1)$ \\
\hline $\mathrm{C}(2)$ & $9500(5)$ & $5508(3)$ & $8726(3)$ & $31(1)$ \\
\hline$C(3)$ & $9342(6)$ & 4751(3) & $8768(4)$ & $44(1)$ \\
\hline $\mathrm{C}(4)$ & $9006(5)$ & $5946(3)$ & 7872(3) & $29(1)$ \\
\hline$C(5)$ & $10003(5)$ & $7421(2)$ & $8681(3)$ & $28(1)$ \\
\hline$C(6)$ & $11226(5)$ & $7482(3)$ & 9401(4) & $41(1)$ \\
\hline$C(7)$ & 10998(5) & $7721(3)$ & $10259(4)$ & $39(1)$ \\
\hline$C(8)$ & $9568(5)$ & $7893(2)$ & $10439(3)$ & $28(1)$ \\
\hline $\mathrm{C}(9)$ & $8362(5)$ & $7834(3)$ & $9702(4)$ & $41(1)$ \\
\hline $\mathrm{C}(10)$ & $8580(5)$ & 7611(3) & $8840(4)$ & $42(1)$ \\
\hline $\mathrm{C}(11)$ & $9279(5)$ & $8119(3)$ & $11369(3)$ & $36(1)$ \\
\hline $\mathrm{C}(12)$ & $8376(6)$ & $5559(3)$ & 6993(3) & $46(1)$ \\
\hline $\mathrm{C}(13)$ & $7385(5)$ & $5864(2)$ & $10367(3)$ & $29(1)$ \\
\hline$C(14)$ & $6325(5)$ & $6131(3)$ & $9656(4)$ & $38(1)$ \\
\hline$C(15)$ & $5363(6)$ & $6661(3)$ & $9846(4)$ & $50(2)$ \\
\hline$C(16)$ & $5447(6)$ & 6917(3) & $10727(4)$ & $46(2)$ \\
\hline $\mathrm{C}(17)$ & $6477(6)$ & $6646(3)$ & 11437(4) & $46(1)$ \\
\hline$C(18)$ & $7428(5)$ & $6124(3)$ & $11262(3)$ & $35(1)$ \\
\hline$C(19)$ & $9968(5)$ & $5265(2)$ & $10693(3)$ & $29(1)$ \\
\hline $\mathrm{C}(20)$ & $10724(5)$ & $5887(3)$ & $10856(3)$ & $38(1)$ \\
\hline $\mathrm{C}(21)$ & $12267(6)$ & 5916(3) & 11199(3) & $47(2)$ \\
\hline $\mathrm{C}(22)$ & 13091(6) & 5321(4) & 11387(4) & $52(2)$ \\
\hline $\mathrm{C}(23)$ & $12360(6)$ & 4699(3) & $11239(3)$ & $48(2)$ \\
\hline $\mathrm{C}(24)$ & $10821(5)$ & 4666(3) & 10902(3) & $36(1)$ \\
\hline$C(25)$ & $7459(5)$ & $4606(2)$ & $10469(3)$ & $23(1)$ \\
\hline$C(26)$ & 7563(5) & $4432(2)$ & $11410(3)$ & $33(1)$ \\
\hline
\end{tabular}




\begin{tabular}{|c|c|c|c|c|}
\hline $\mathrm{C}(27)$ & $6707(5)$ & $3903(3)$ & $11670(3)$ & $34(1)$ \\
\hline$C(28)$ & $5731(5)$ & $3545(3)$ & $11027(3)$ & $32(1)$ \\
\hline $\mathrm{C}(29)$ & $5588(5)$ & $3729(2)$ & $10102(3)$ & $34(1)$ \\
\hline $\mathrm{C}(30)$ & $6450(5)$ & $4262(2)$ & $9822(3)$ & $33(1)$ \\
\hline$S(1 \mathrm{~A})$ & $5194(1)$ & $3454(1)$ & $7346(1)$ & $35(1)$ \\
\hline $\mathrm{N}(1 \mathrm{~A})$ & 2916(4) & $5491(2)$ & $5830(2)$ & $29(1)$ \\
\hline $\mathrm{O}(1 \mathrm{~A})$ & $5247(4)$ & $4250(2)$ & $7382(2)$ & $36(1)$ \\
\hline $\mathrm{O}(2 \mathrm{~A})$ & $3838(4)$ & $3222(2)$ & $7658(2)$ & $49(1)$ \\
\hline $\mathrm{O}(3 \mathrm{~A})$ & $6629(4)$ & $3247(2)$ & $7835(2)$ & $51(1)$ \\
\hline $\mathrm{C}(1 \mathrm{~A})$ & $2672(5)$ & $5489(2)$ & $4787(3)$ & $28(1)$ \\
\hline $\mathrm{C}(2 \mathrm{~A})$ & $4176(6)$ & $5873(3)$ & $6359(3)$ & $37(1)$ \\
\hline $\mathrm{C}(3 \mathrm{~A})$ & $4209(5)$ & $5117(2)$ & 6329(3) & $30(1)$ \\
\hline $\mathrm{C}(4 \mathrm{~A})$ & $3841(5)$ & $4633(3)$ & $7077(3)$ & $36(1)$ \\
\hline $\mathrm{C}(5 \mathrm{~A})$ & $5103(5)$ & $3243(2)$ & $6169(3)$ & $30(1)$ \\
\hline $\mathrm{C}(6 \mathrm{~A})$ & $6412(5)$ & $3114(3)$ & $5797(3)$ & $35(1)$ \\
\hline $\mathrm{C}(7 \mathrm{~A})$ & $6323(5)$ & 2937(3) & $4873(3)$ & $34(1)$ \\
\hline $\mathrm{C}(8 \mathrm{~A})$ & $4940(5)$ & $2900(2)$ & 4304(3) & $29(1)$ \\
\hline $\mathrm{C}(9 \mathrm{~A})$ & $3626(5)$ & $3017(2)$ & $4687(3)$ & $33(1)$ \\
\hline $\mathrm{C}(10 \mathrm{~A})$ & $3699(5)$ & $3183(2)$ & 5611(3) & $31(1)$ \\
\hline $\mathrm{C}(11 \mathrm{~A})$ & $4827(6)$ & 2734(3) & $3289(3)$ & $38(1)$ \\
\hline $\mathrm{C}(12 \mathrm{~A})$ & $3323(6)$ & 4972(3) & 7907(3) & $44(1)$ \\
\hline $\mathrm{C}(13 \mathrm{~A})$ & $1690(5)$ & $6107(2)$ & $4460(3)$ & $28(1)$ \\
\hline $\mathrm{C}(14 \mathrm{~A})$ & $1470(5)$ & $6284(2)$ & $3516(3)$ & $33(1)$ \\
\hline $\mathrm{C}(15 \mathrm{~A})$ & $528(5)$ & 6811(3) & $3183(3)$ & $38(1)$ \\
\hline $\mathrm{C}(16 \mathrm{~A})$ & $-197(5)$ & $7190(3)$ & $3783(3)$ & $37(1)$ \\
\hline $\mathrm{C}(17 \mathrm{~A})$ & $4(5)$ & 7023(3) & $4707(3)$ & $33(1)$ \\
\hline $\mathrm{C}(18 \mathrm{~A})$ & $928(5)$ & $6489(3)$ & $5050(3)$ & $34(1)$ \\
\hline $\mathrm{C}(19 \mathrm{~A})$ & $4179(5)$ & $5504(3)$ & 4399(3) & $29(1)$ \\
\hline $\mathrm{C}(20 \mathrm{~A})$ & $4906(5)$ & $6110(3)$ & $4275(3)$ & $36(1)$ \\
\hline $\mathrm{C}(21 \mathrm{~A})$ & $6337(6)$ & $6130(3)$ & 4014(4) & $45(1)$ \\
\hline $\mathrm{C}(22 \mathrm{~A})$ & $7085(6)$ & $5527(4)$ & $3883(4)$ & $52(2)$ \\
\hline $\mathrm{C}(23 \mathrm{~A})$ & $6381(5)$ & 4915(3) & 4002(3) & $43(1)$ \\
\hline $\mathrm{C}(24 \mathrm{~A})$ & $4955(5)$ & $4896(3)$ & $4263(3)$ & $35(1)$ \\
\hline $\mathrm{C}(25 \mathrm{~A})$ & 1693(5) & $4851(2)$ & 4492(3) & $26(1)$ \\
\hline $\mathrm{C}(26 \mathrm{~A})$ & $1573(5)$ & $4597(2)$ & $3592(3)$ & $30(1)$ \\
\hline $\mathrm{C}(27 \mathrm{~A})$ & $617(6)$ & $4047(3)$ & $3323(4)$ & $37(1)$ \\
\hline
\end{tabular}




\begin{tabular}{lrlll}
$\mathrm{C}(28 \mathrm{~A})$ & $-214(5)$ & $3753(3)$ & $3934(3)$ & $35(1)$ \\
$\mathrm{C}(29 \mathrm{~A})$ & $-122(5)$ & $4006(3)$ & $4822(4)$ & $35(1)$ \\
$\mathrm{C}(30 \mathrm{~A})$ & $822(5)$ & $4552(2)$ & $5102(3)$ & $29(1)$ \\
\hline
\end{tabular}


Table 3. Bond lengths [ $\left[\AA\right.$ ] and angles $\left[{ }^{\circ}\right]$ for 25.

\begin{tabular}{|c|c|}
\hline $\mathrm{S}(1)-\mathrm{O}(3)$ & $1.434(3)$ \\
\hline $\mathrm{S}(1)-\mathrm{O}(2)$ & $1.435(3)$ \\
\hline $\mathrm{S}(1)-\mathrm{O}(1)$ & $1.571(3)$ \\
\hline$S(1)-C(5)$ & $1.754(5)$ \\
\hline $\mathrm{N}(1)-\mathrm{C}(2)$ & $1.449(5)$ \\
\hline $\mathrm{N}(1)-\mathrm{C}(3)$ & $1.454(6)$ \\
\hline $\mathrm{N}(1)-\mathrm{C}(1)$ & $1.512(5)$ \\
\hline $\mathrm{O}(1)-\mathrm{C}(4)$ & $1.476(5)$ \\
\hline$C(1)-C(19)$ & $1.538(6)$ \\
\hline$C(1)-C(25)$ & $1.546(6)$ \\
\hline$C(1)-C(13)$ & $1.543(6)$ \\
\hline $\mathrm{C}(2)-\mathrm{C}(3)$ & $1.494(7)$ \\
\hline $\mathrm{C}(2)-\mathrm{C}(4)$ & $1.520(6)$ \\
\hline $\mathrm{C}(2)-\mathrm{H}(2)$ & 1.0000 \\
\hline $\mathrm{C}(3)-\mathrm{H}(3 \mathrm{~A})$ & 0.9900 \\
\hline $\mathrm{C}(3)-\mathrm{H}(3 \mathrm{~B})$ & 0.9900 \\
\hline$C(4)-C(12)$ & $1.522(7)$ \\
\hline $\mathrm{C}(4)-\mathrm{H}(4)$ & 1.0000 \\
\hline$C(5)-C(10)$ & $1.380(6)$ \\
\hline $\mathrm{C}(5)-\mathrm{C}(6)$ & $1.399(7)$ \\
\hline$C(6)-C(7)$ & $1.380(7)$ \\
\hline $\mathrm{C}(6)-\mathrm{H}(6)$ & 0.9500 \\
\hline $\mathrm{C}(7)-\mathrm{C}(8)$ & $1.386(6)$ \\
\hline $\mathrm{C}(7)-\mathrm{H}(7)$ & 0.9500 \\
\hline $\mathrm{C}(8)-\mathrm{C}(9)$ & $1.405(7)$ \\
\hline $\mathrm{C}(8)-\mathrm{C}(11)$ & $1.484(7)$ \\
\hline C(9)-C(10) & $1.372(7)$ \\
\hline $\mathrm{C}(9)-\mathrm{H}(9)$ & 0.9500 \\
\hline $\mathrm{C}(10)-\mathrm{H}(10)$ & 0.9500 \\
\hline $\mathrm{C}(11)-\mathrm{H}(11 \mathrm{~A})$ & 0.9800 \\
\hline $\mathrm{C}(11)-\mathrm{H}(11 \mathrm{~B})$ & 0.9800 \\
\hline $\mathrm{C}(11)-\mathrm{H}(11 \mathrm{C})$ & 0.9800 \\
\hline $\mathrm{C}(12)-\mathrm{H}(12 \mathrm{~A})$ & 0.9800 \\
\hline $\mathrm{C}(12)-\mathrm{H}(12 \mathrm{~B})$ & 0.9800 \\
\hline
\end{tabular}




\begin{tabular}{|c|c|}
\hline $\mathrm{C}(12)-\mathrm{H}(12 \mathrm{C})$ & 0.9800 \\
\hline$C(13)-C(14)$ & $1.394(7)$ \\
\hline $\mathrm{C}(13)-\mathrm{C}(18)$ & $1.395(6)$ \\
\hline$C(14)-C(15)$ & $1.406(7)$ \\
\hline $\mathrm{C}(14)-\mathrm{H}(14)$ & 0.9500 \\
\hline$C(15)-C(16)$ & $1.369(7)$ \\
\hline $\mathrm{C}(15)-\mathrm{H}(15)$ & 0.9500 \\
\hline$C(16)-C(17)$ & $1.381(8)$ \\
\hline $\mathrm{C}(16)-\mathrm{H}(16)$ & 0.9500 \\
\hline $\mathrm{C}(17)-\mathrm{C}(18)$ & $1.381(7)$ \\
\hline $\mathrm{C}(17)-\mathrm{H}(17)$ & 0.9500 \\
\hline $\mathrm{C}(18)-\mathrm{H}(18)$ & 0.9500 \\
\hline $\mathrm{C}(19)-\mathrm{C}(20)$ & $1.400(6)$ \\
\hline C(19)-C(24) & $1.408(7)$ \\
\hline$C(20)-C(21)$ & $1.395(7)$ \\
\hline $\mathrm{C}(20)-\mathrm{H}(20)$ & 0.9500 \\
\hline $\mathrm{C}(21)-\mathrm{C}(22)$ & $1.386(8)$ \\
\hline $\mathrm{C}(21)-\mathrm{H}(21)$ & 0.9500 \\
\hline $\mathrm{C}(22)-\mathrm{C}(23)$ & $1.386(8)$ \\
\hline $\mathrm{C}(22)-\mathrm{H}(22)$ & 0.9500 \\
\hline $\mathrm{C}(23)-\mathrm{C}(24)$ & $1.391(7)$ \\
\hline $\mathrm{C}(23)-\mathrm{H}(23)$ & 0.9500 \\
\hline $\mathrm{C}(24)-\mathrm{H}(24)$ & 0.9500 \\
\hline $\mathrm{C}(25)-\mathrm{C}(30)$ & $1.378(6)$ \\
\hline$C(25)-C(26)$ & $1.401(6)$ \\
\hline $\mathrm{C}(26)-\mathrm{C}(27)$ & $1.377(6)$ \\
\hline C(26)-H(26) & 0.9500 \\
\hline C(27)-C(28) & $1.373(6)$ \\
\hline $\mathrm{C}(27)-\mathrm{H}(27)$ & 0.9500 \\
\hline C(28)-C(29) & $1.383(6)$ \\
\hline C(28)-H(28) & 0.9500 \\
\hline C(29)-C(30) & $1.397(6)$ \\
\hline C(29)-H(29) & 0.9500 \\
\hline $\mathrm{C}(30)-\mathrm{H}(30)$ & 0.9500 \\
\hline $\mathrm{S}(1 \mathrm{~A})-\mathrm{O}(3 \mathrm{~A})$ & $1.429(3)$ \\
\hline $\mathrm{S}(1 \mathrm{~A})-\mathrm{O}(2 \mathrm{~A})$ & $1.434(4)$ \\
\hline
\end{tabular}




\begin{tabular}{|c|c|}
\hline $\mathrm{S}(1 \mathrm{~A})-\mathrm{O}(1 \mathrm{~A})$ & $1.564(4)$ \\
\hline$S(1 \mathrm{~A})-C(5 \mathrm{~A})$ & $1.753(5)$ \\
\hline $\mathrm{N}(1 \mathrm{~A})-\mathrm{C}(3 \mathrm{~A})$ & $1.465(5)$ \\
\hline $\mathrm{N}(1 \mathrm{~A})-\mathrm{C}(2 \mathrm{~A})$ & $1.470(6)$ \\
\hline $\mathrm{N}(1 \mathrm{~A})-\mathrm{C}(1 \mathrm{~A})$ & $1.502(5)$ \\
\hline $\mathrm{O}(1 \mathrm{~A})-\mathrm{C}(4 \mathrm{~A})$ & $1.475(5)$ \\
\hline$C(1 \mathrm{~A})-\mathrm{C}(13 \mathrm{~A})$ & $1.531(6)$ \\
\hline$C(1 \mathrm{~A})-\mathrm{C}(19 \mathrm{~A})$ & $1.540(6)$ \\
\hline$C(1 \mathrm{~A})-\mathrm{C}(25 \mathrm{~A})$ & $1.551(6)$ \\
\hline $\mathrm{C}(2 \mathrm{~A})-\mathrm{C}(3 \mathrm{~A})$ & $1.485(6)$ \\
\hline $\mathrm{C}(2 \mathrm{~A})-\mathrm{H}(2 \mathrm{~A} 1)$ & 0.9900 \\
\hline $\mathrm{C}(2 \mathrm{~A})-\mathrm{H}(2 \mathrm{~A} 2)$ & 0.9900 \\
\hline $\mathrm{C}(3 \mathrm{~A})-\mathrm{C}(4 \mathrm{~A})$ & $1.519(6)$ \\
\hline $\mathrm{C}(3 \mathrm{~A})-\mathrm{H}(3 \mathrm{~A} 1)$ & 1.0000 \\
\hline $\mathrm{C}(4 \mathrm{~A})-\mathrm{C}(12 \mathrm{~A})$ & $1.513(7)$ \\
\hline $\mathrm{C}(4 \mathrm{~A})-\mathrm{H}(4 \mathrm{~A})$ & 1.0000 \\
\hline$C(5 A)-C(6 A)$ & $1.388(6)$ \\
\hline $\mathrm{C}(5 \mathrm{~A})-\mathrm{C}(10 \mathrm{~A})$ & $1.393(6)$ \\
\hline $\mathrm{C}(6 \mathrm{~A})-\mathrm{C}(7 \mathrm{~A})$ & $1.380(7)$ \\
\hline $\mathrm{C}(6 \mathrm{~A})-\mathrm{H}(6 \mathrm{~A})$ & 0.9500 \\
\hline $\mathrm{C}(7 \mathrm{~A})-\mathrm{C}(8 \mathrm{~A})$ & $1.381(6)$ \\
\hline $\mathrm{C}(7 \mathrm{~A})-\mathrm{H}(7 \mathrm{~A})$ & 0.9500 \\
\hline $\mathrm{C}(8 \mathrm{~A})-\mathrm{C}(9 \mathrm{~A})$ & $1.395(6)$ \\
\hline $\mathrm{C}(8 \mathrm{~A})-\mathrm{C}(11 \mathrm{~A})$ & $1.502(6)$ \\
\hline $\mathrm{C}(9 \mathrm{~A})-\mathrm{C}(10 \mathrm{~A})$ & $1.376(6)$ \\
\hline C(9A)-H(9A) & 0.9500 \\
\hline $\mathrm{C}(10 \mathrm{~A})-\mathrm{H}(10 \mathrm{~A})$ & 0.9500 \\
\hline $\mathrm{C}(11 \mathrm{~A})-\mathrm{H}(11 \mathrm{D})$ & 0.9800 \\
\hline $\mathrm{C}(11 \mathrm{~A})-\mathrm{H}(11 \mathrm{E})$ & 0.9800 \\
\hline $\mathrm{C}(11 \mathrm{~A})-\mathrm{H}(11 \mathrm{~F})$ & 0.9800 \\
\hline $\mathrm{C}(12 \mathrm{~A})-\mathrm{H}(12 \mathrm{D})$ & 0.9800 \\
\hline $\mathrm{C}(12 \mathrm{~A})-\mathrm{H}(12 \mathrm{E})$ & 0.9800 \\
\hline $\mathrm{C}(12 \mathrm{~A})-\mathrm{H}(12 \mathrm{~F})$ & 0.9800 \\
\hline $\mathrm{C}(13 \mathrm{~A})-\mathrm{C}(18 \mathrm{~A})$ & $1.395(6)$ \\
\hline $\mathrm{C}(13 \mathrm{~A})-\mathrm{C}(14 \mathrm{~A})$ & $1.402(6)$ \\
\hline$C(14 A)-C(15 A)$ & $1.375(6)$ \\
\hline
\end{tabular}




\begin{tabular}{|c|c|}
\hline $\mathrm{C}(14 \mathrm{~A})-\mathrm{H}(14 \mathrm{~A})$ & 0.9500 \\
\hline$C(15 A)-C(16 A)$ & $1.383(7)$ \\
\hline $\mathrm{C}(15 \mathrm{~A})-\mathrm{H}(15 \mathrm{~A})$ & 0.9500 \\
\hline$C(16 A)-C(17 A)$ & $1.370(6)$ \\
\hline$C(16 A)-H(16 A)$ & 0.9500 \\
\hline $\mathrm{C}(17 \mathrm{~A})-\mathrm{C}(18 \mathrm{~A})$ & $1.381(7)$ \\
\hline $\mathrm{C}(17 \mathrm{~A})-\mathrm{H}(17 \mathrm{~A})$ & 0.9500 \\
\hline $\mathrm{C}(18 \mathrm{~A})-\mathrm{H}(18 \mathrm{~A})$ & 0.9500 \\
\hline$C(19 A)-C(20 A)$ & $1.381(7)$ \\
\hline$C(19 A)-C(24 A)$ & $1.410(7)$ \\
\hline$C(20 A)-C(21 A)$ & $1.391(7)$ \\
\hline $\mathrm{C}(20 \mathrm{~A})-\mathrm{H}(20 \mathrm{~A})$ & 0.9500 \\
\hline$C(21 \mathrm{~A})-\mathrm{C}(22 \mathrm{~A})$ & $1.387(8)$ \\
\hline $\mathrm{C}(21 \mathrm{~A})-\mathrm{H}(21 \mathrm{~A})$ & 0.9500 \\
\hline$C(22 A)-C(23 A)$ & $1.380(8)$ \\
\hline $\mathrm{C}(22 \mathrm{~A})-\mathrm{H}(22 \mathrm{~A})$ & 0.9500 \\
\hline$C(23 \mathrm{~A})-\mathrm{C}(24 \mathrm{~A})$ & $1.386(7)$ \\
\hline $\mathrm{C}(23 \mathrm{~A})-\mathrm{H}(23 \mathrm{~A})$ & 0.9500 \\
\hline $\mathrm{C}(24 \mathrm{~A})-\mathrm{H}(24 \mathrm{~A})$ & 0.9500 \\
\hline$C(25 A)-C(26 A)$ & $1.392(6)$ \\
\hline$C(25 A)-C(30 A)$ & $1.398(6)$ \\
\hline$C(26 A)-C(27 A)$ & $1.396(7)$ \\
\hline $\mathrm{C}(26 \mathrm{~A})-\mathrm{H}(26 \mathrm{~A})$ & 0.9500 \\
\hline $\mathrm{C}(27 \mathrm{~A})-\mathrm{C}(28 \mathrm{~A})$ & $1.372(7)$ \\
\hline $\mathrm{C}(27 \mathrm{~A})-\mathrm{H}(27 \mathrm{~A})$ & 0.9500 \\
\hline $\mathrm{C}(28 \mathrm{~A})-\mathrm{C}(29 \mathrm{~A})$ & $1.377(7)$ \\
\hline $\mathrm{C}(28 \mathrm{~A})-\mathrm{H}(28 \mathrm{~A})$ & 0.9500 \\
\hline$C(29 A)-C(30 A)$ & $1.386(7)$ \\
\hline $\mathrm{C}(29 \mathrm{~A})-\mathrm{H}(29 \mathrm{~A})$ & 0.9500 \\
\hline $\mathrm{C}(30 \mathrm{~A})-\mathrm{H}(30 \mathrm{~A})$ & 0.9500 \\
\hline $\mathrm{O}(3)-\mathrm{S}(1)-\mathrm{O}(2)$ & $119.2(2)$ \\
\hline $\mathrm{O}(3)-\mathrm{S}(1)-\mathrm{O}(1)$ & $104.3(2)$ \\
\hline $\mathrm{O}(2)-\mathrm{S}(1)-\mathrm{O}(1)$ & 109.6(2) \\
\hline $\mathrm{O}(3)-\mathrm{S}(1)-\mathrm{C}(5)$ & $109.8(2)$ \\
\hline $\mathrm{O}(2)-\mathrm{S}(1)-\mathrm{C}(5)$ & $107.9(2)$ \\
\hline
\end{tabular}




\begin{tabular}{|c|c|}
\hline $\mathrm{O}(1)-\mathrm{S}(1)-\mathrm{C}(5)$ & $105.0(2)$ \\
\hline $\mathrm{C}(2)-\mathrm{N}(1)-\mathrm{C}(3)$ & $62.0(3)$ \\
\hline $\mathrm{C}(2)-\mathrm{N}(1)-\mathrm{C}(1)$ & $120.5(3)$ \\
\hline $\mathrm{C}(3)-\mathrm{N}(1)-\mathrm{C}(1)$ & $119.6(4)$ \\
\hline $\mathrm{C}(4)-\mathrm{O}(1)-\mathrm{S}(1)$ & $117.5(3)$ \\
\hline $\mathrm{N}(1)-\mathrm{C}(1)-\mathrm{C}(19)$ & $110.4(3)$ \\
\hline $\mathrm{N}(1)-\mathrm{C}(1)-\mathrm{C}(25)$ & $107.4(3)$ \\
\hline$C(19)-C(1)-C(25)$ & $112.7(4)$ \\
\hline $\mathrm{N}(1)-\mathrm{C}(1)-\mathrm{C}(13)$ & 106.1(3) \\
\hline $\mathrm{C}(19)-\mathrm{C}(1)-\mathrm{C}(13)$ & $113.2(4)$ \\
\hline $\mathrm{C}(25)-\mathrm{C}(1)-\mathrm{C}(13)$ & $106.5(3)$ \\
\hline $\mathrm{N}(1)-\mathrm{C}(2)-\mathrm{C}(3)$ & $59.2(3)$ \\
\hline $\mathrm{N}(1)-\mathrm{C}(2)-\mathrm{C}(4)$ & $116.2(4)$ \\
\hline$C(3)-C(2)-C(4)$ & $125.3(5)$ \\
\hline $\mathrm{N}(1)-\mathrm{C}(2)-\mathrm{H}(2)$ & 114.7 \\
\hline $\mathrm{C}(3)-\mathrm{C}(2)-\mathrm{H}(2)$ & 114.7 \\
\hline $\mathrm{C}(4)-\mathrm{C}(2)-\mathrm{H}(2)$ & 114.7 \\
\hline $\mathrm{N}(1)-\mathrm{C}(3)-\mathrm{C}(2)$ & $58.9(3)$ \\
\hline $\mathrm{N}(1)-\mathrm{C}(3)-\mathrm{H}(3 \mathrm{~A})$ & 117.9 \\
\hline $\mathrm{C}(2)-\mathrm{C}(3)-\mathrm{H}(3 \mathrm{~A})$ & 117.9 \\
\hline $\mathrm{N}(1)-\mathrm{C}(3)-\mathrm{H}(3 \mathrm{~B})$ & 117.9 \\
\hline $\mathrm{C}(2)-\mathrm{C}(3)-\mathrm{H}(3 \mathrm{~B})$ & 117.9 \\
\hline $\mathrm{H}(3 \mathrm{~A})-\mathrm{C}(3)-\mathrm{H}(3 \mathrm{~B})$ & 115.0 \\
\hline $\mathrm{O}(1)-\mathrm{C}(4)-\mathrm{C}(2)$ & $104.2(3)$ \\
\hline $\mathrm{O}(1)-\mathrm{C}(4)-\mathrm{C}(12)$ & $109.4(4)$ \\
\hline $\mathrm{C}(2)-\mathrm{C}(4)-\mathrm{C}(12)$ & $115.5(4)$ \\
\hline $\mathrm{O}(1)-\mathrm{C}(4)-\mathrm{H}(4)$ & 109.2 \\
\hline $\mathrm{C}(2)-\mathrm{C}(4)-\mathrm{H}(4)$ & 109.2 \\
\hline $\mathrm{C}(12)-\mathrm{C}(4)-\mathrm{H}(4)$ & 109.2 \\
\hline$C(10)-C(5)-C(6)$ & $119.1(5)$ \\
\hline $\mathrm{C}(10)-\mathrm{C}(5)-\mathrm{S}(1)$ & $120.9(4)$ \\
\hline$C(6)-C(5)-S(1)$ & $120.0(3)$ \\
\hline$C(7)-C(6)-C(5)$ & $120.0(4)$ \\
\hline $\mathrm{C}(7)-\mathrm{C}(6)-\mathrm{H}(6)$ & 120.0 \\
\hline $\mathrm{C}(5)-\mathrm{C}(6)-\mathrm{H}(6)$ & 120.0 \\
\hline $\mathrm{C}(6)-\mathrm{C}(7)-\mathrm{C}(8)$ & $121.6(4)$ \\
\hline
\end{tabular}




\begin{tabular}{|c|c|}
\hline $\mathrm{C}(6)-\mathrm{C}(7)-\mathrm{H}(7)$ & 119.2 \\
\hline $\mathrm{C}(8)-\mathrm{C}(7)-\mathrm{H}(7)$ & 119.2 \\
\hline $\mathrm{C}(7)-\mathrm{C}(8)-\mathrm{C}(9)$ & $117.3(4)$ \\
\hline $\mathrm{C}(7)-\mathrm{C}(8)-\mathrm{C}(11)$ & $122.7(4)$ \\
\hline$C(9)-C(8)-C(11)$ & $120.0(4)$ \\
\hline$C(10)-C(9)-C(8)$ & $121.7(4)$ \\
\hline $\mathrm{C}(10)-\mathrm{C}(9)-\mathrm{H}(9)$ & 119.2 \\
\hline $\mathrm{C}(8)-\mathrm{C}(9)-\mathrm{H}(9)$ & 119.2 \\
\hline$C(9)-C(10)-C(5)$ & $120.3(4)$ \\
\hline $\mathrm{C}(9)-\mathrm{C}(10)-\mathrm{H}(10)$ & 119.9 \\
\hline $\mathrm{C}(5)-\mathrm{C}(10)-\mathrm{H}(10)$ & 119.9 \\
\hline $\mathrm{C}(8)-\mathrm{C}(11)-\mathrm{H}(11 \mathrm{~A})$ & 109.5 \\
\hline $\mathrm{C}(8)-\mathrm{C}(11)-\mathrm{H}(11 \mathrm{~B})$ & 109.5 \\
\hline $\mathrm{H}(11 \mathrm{~A})-\mathrm{C}(11)-\mathrm{H}(11 \mathrm{~B})$ & 109.5 \\
\hline $\mathrm{C}(8)-\mathrm{C}(11)-\mathrm{H}(11 \mathrm{C})$ & 109.5 \\
\hline $\mathrm{H}(11 \mathrm{~A})-\mathrm{C}(11)-\mathrm{H}(11 \mathrm{C})$ & 109.5 \\
\hline $\mathrm{H}(11 \mathrm{~B})-\mathrm{C}(11)-\mathrm{H}(11 \mathrm{C})$ & 109.5 \\
\hline $\mathrm{C}(4)-\mathrm{C}(12)-\mathrm{H}(12 \mathrm{~A})$ & 109.5 \\
\hline $\mathrm{C}(4)-\mathrm{C}(12)-\mathrm{H}(12 \mathrm{~B})$ & 109.5 \\
\hline $\mathrm{H}(12 \mathrm{~A})-\mathrm{C}(12)-\mathrm{H}(12 \mathrm{~B})$ & 109.5 \\
\hline $\mathrm{C}(4)-\mathrm{C}(12)-\mathrm{H}(12 \mathrm{C})$ & 109.5 \\
\hline $\mathrm{H}(12 \mathrm{~A})-\mathrm{C}(12)-\mathrm{H}(12 \mathrm{C})$ & 109.5 \\
\hline $\mathrm{H}(12 \mathrm{~B})-\mathrm{C}(12)-\mathrm{H}(12 \mathrm{C})$ & 109.5 \\
\hline $\mathrm{C}(14)-\mathrm{C}(13)-\mathrm{C}(18)$ & $118.3(4)$ \\
\hline$C(14)-C(13)-C(1)$ & $120.3(4)$ \\
\hline $\mathrm{C}(18)-\mathrm{C}(13)-\mathrm{C}(1)$ & $120.9(4)$ \\
\hline $\mathrm{C}(13)-\mathrm{C}(14)-\mathrm{C}(15)$ & $120.0(5)$ \\
\hline $\mathrm{C}(13)-\mathrm{C}(14)-\mathrm{H}(14)$ & 120.0 \\
\hline $\mathrm{C}(15)-\mathrm{C}(14)-\mathrm{H}(14)$ & 120.0 \\
\hline$C(16)-C(15)-C(14)$ & $120.6(6)$ \\
\hline $\mathrm{C}(16)-\mathrm{C}(15)-\mathrm{H}(15)$ & 119.7 \\
\hline $\mathrm{C}(14)-\mathrm{C}(15)-\mathrm{H}(15)$ & 119.7 \\
\hline$C(15)-C(16)-C(17)$ & $119.7(5)$ \\
\hline $\mathrm{C}(15)-\mathrm{C}(16)-\mathrm{H}(16)$ & 120.1 \\
\hline $\mathrm{C}(17)-\mathrm{C}(16)-\mathrm{H}(16)$ & 120.1 \\
\hline $\mathrm{C}(18)-\mathrm{C}(17)-\mathrm{C}(16)$ & $120.4(5)$ \\
\hline
\end{tabular}




\begin{tabular}{|c|c|}
\hline $\mathrm{C}(18)-\mathrm{C}(17)-\mathrm{H}(17)$ & 119.8 \\
\hline $\mathrm{C}(16)-\mathrm{C}(17)-\mathrm{H}(17)$ & 119.8 \\
\hline $\mathrm{C}(17)-\mathrm{C}(18)-\mathrm{C}(13)$ & $121.0(5)$ \\
\hline $\mathrm{C}(17)-\mathrm{C}(18)-\mathrm{H}(18)$ & 119.5 \\
\hline $\mathrm{C}(13)-\mathrm{C}(18)-\mathrm{H}(18)$ & 119.5 \\
\hline $\mathrm{C}(20)-\mathrm{C}(19)-\mathrm{C}(24)$ & $117.4(4)$ \\
\hline$C(20)-C(19)-C(1)$ & $121.1(4)$ \\
\hline$C(24)-C(19)-C(1)$ & $121.1(4)$ \\
\hline$C(21)-C(20)-C(19)$ & $121.4(5)$ \\
\hline $\mathrm{C}(21)-\mathrm{C}(20)-\mathrm{H}(20)$ & 119.3 \\
\hline $\mathrm{C}(19)-\mathrm{C}(20)-\mathrm{H}(20)$ & 119.3 \\
\hline $\mathrm{C}(22)-\mathrm{C}(21)-\mathrm{C}(20)$ & $120.3(6)$ \\
\hline $\mathrm{C}(22)-\mathrm{C}(21)-\mathrm{H}(21)$ & 119.9 \\
\hline $\mathrm{C}(20)-\mathrm{C}(21)-\mathrm{H}(21)$ & 119.9 \\
\hline $\mathrm{C}(21)-\mathrm{C}(22)-\mathrm{C}(23)$ & $119.2(5)$ \\
\hline $\mathrm{C}(21)-\mathrm{C}(22)-\mathrm{H}(22)$ & 120.4 \\
\hline $\mathrm{C}(23)-\mathrm{C}(22)-\mathrm{H}(22)$ & 120.4 \\
\hline $\mathrm{C}(22)-\mathrm{C}(23)-\mathrm{C}(24)$ & $120.9(6)$ \\
\hline $\mathrm{C}(22)-\mathrm{C}(23)-\mathrm{H}(23)$ & 119.6 \\
\hline $\mathrm{C}(24)-\mathrm{C}(23)-\mathrm{H}(23)$ & 119.6 \\
\hline $\mathrm{C}(23)-\mathrm{C}(24)-\mathrm{C}(19)$ & $120.8(5)$ \\
\hline $\mathrm{C}(23)-\mathrm{C}(24)-\mathrm{H}(24)$ & 119.6 \\
\hline $\mathrm{C}(19)-\mathrm{C}(24)-\mathrm{H}(24)$ & 119.6 \\
\hline $\mathrm{C}(30)-\mathrm{C}(25)-\mathrm{C}(26)$ & $119.1(4)$ \\
\hline $\mathrm{C}(30)-\mathrm{C}(25)-\mathrm{C}(1)$ & $120.9(4)$ \\
\hline $\mathrm{C}(26)-\mathrm{C}(25)-\mathrm{C}(1)$ & $119.6(4)$ \\
\hline $\mathrm{C}(27)-\mathrm{C}(26)-\mathrm{C}(25)$ & $119.8(4)$ \\
\hline $\mathrm{C}(27)-\mathrm{C}(26)-\mathrm{H}(26)$ & 120.1 \\
\hline $\mathrm{C}(25)-\mathrm{C}(26)-\mathrm{H}(26)$ & 120.1 \\
\hline$C(28)-C(27)-C(26)$ & $121.4(4)$ \\
\hline $\mathrm{C}(28)-\mathrm{C}(27)-\mathrm{H}(27)$ & 119.3 \\
\hline $\mathrm{C}(26)-\mathrm{C}(27)-\mathrm{H}(27)$ & 119.3 \\
\hline $\mathrm{C}(27)-\mathrm{C}(28)-\mathrm{C}(29)$ & $119.0(4)$ \\
\hline $\mathrm{C}(27)-\mathrm{C}(28)-\mathrm{H}(28)$ & 120.5 \\
\hline $\mathrm{C}(29)-\mathrm{C}(28)-\mathrm{H}(28)$ & 120.5 \\
\hline$C(28)-C(29)-C(30)$ & $120.5(4)$ \\
\hline
\end{tabular}




\begin{tabular}{|c|c|}
\hline $\mathrm{C}(28)-\mathrm{C}(29)-\mathrm{H}(29)$ & 119.7 \\
\hline $\mathrm{C}(30)-\mathrm{C}(29)-\mathrm{H}(29)$ & 119.7 \\
\hline$C(25)-C(30)-C(29)$ & $120.1(4)$ \\
\hline $\mathrm{C}(25)-\mathrm{C}(30)-\mathrm{H}(30)$ & 120.0 \\
\hline $\mathrm{C}(29)-\mathrm{C}(30)-\mathrm{H}(30)$ & 120.0 \\
\hline $\mathrm{O}(3 \mathrm{~A})-\mathrm{S}(1 \mathrm{~A})-\mathrm{O}(2 \mathrm{~A})$ & $119.4(2)$ \\
\hline $\mathrm{O}(3 \mathrm{~A})-\mathrm{S}(1 \mathrm{~A})-\mathrm{O}(1 \mathrm{~A})$ & $104.3(2)$ \\
\hline $\mathrm{O}(2 \mathrm{~A})-\mathrm{S}(1 \mathrm{~A})-\mathrm{O}(1 \mathrm{~A})$ & $109.3(2)$ \\
\hline $\mathrm{O}(3 \mathrm{~A})-\mathrm{S}(1 \mathrm{~A})-\mathrm{C}(5 \mathrm{~A})$ & $108.8(2)$ \\
\hline $\mathrm{O}(2 \mathrm{~A})-\mathrm{S}(1 \mathrm{~A})-\mathrm{C}(5 \mathrm{~A})$ & $108.7(2)$ \\
\hline $\mathrm{O}(1 \mathrm{~A})-\mathrm{S}(1 \mathrm{~A})-\mathrm{C}(5 \mathrm{~A})$ & $105.4(2)$ \\
\hline$C(3 \mathrm{~A})-\mathrm{N}(1 \mathrm{~A})-\mathrm{C}(2 \mathrm{~A})$ & $60.8(3)$ \\
\hline$C(3 A)-N(1 A)-C(1 A)$ & $118.8(3)$ \\
\hline$C(2 A)-N(1 A)-C(1 A)$ & $120.8(4)$ \\
\hline $\mathrm{C}(4 \mathrm{~A})-\mathrm{O}(1 \mathrm{~A})-\mathrm{S}(1 \mathrm{~A})$ & 118.7(3) \\
\hline $\mathrm{N}(1 \mathrm{~A})-\mathrm{C}(1 \mathrm{~A})-\mathrm{C}(13 \mathrm{~A})$ & $107.5(3)$ \\
\hline$N(1 A)-C(1 A)-C(19 A)$ & $111.8(3)$ \\
\hline$C(13 A)-C(1 A)-C(19 A)$ & $111.4(4)$ \\
\hline $\mathrm{N}(1 \mathrm{~A})-\mathrm{C}(1 \mathrm{~A})-\mathrm{C}(25 \mathrm{~A})$ & $105.7(4)$ \\
\hline$C(13 \mathrm{~A})-\mathrm{C}(1 \mathrm{~A})-\mathrm{C}(25 \mathrm{~A})$ & $106.3(3)$ \\
\hline$C(19 A)-C(1 A)-C(25 A)$ & $113.8(4)$ \\
\hline $\mathrm{N}(1 \mathrm{~A})-\mathrm{C}(2 \mathrm{~A})-\mathrm{C}(3 \mathrm{~A})$ & $59.4(3)$ \\
\hline $\mathrm{N}(1 \mathrm{~A})-\mathrm{C}(2 \mathrm{~A})-\mathrm{H}(2 \mathrm{~A} 1)$ & 117.8 \\
\hline $\mathrm{C}(3 \mathrm{~A})-\mathrm{C}(2 \mathrm{~A})-\mathrm{H}(2 \mathrm{~A} 1)$ & 117.8 \\
\hline $\mathrm{N}(1 \mathrm{~A})-\mathrm{C}(2 \mathrm{~A})-\mathrm{H}(2 \mathrm{~A} 2)$ & 117.8 \\
\hline $\mathrm{C}(3 \mathrm{~A})-\mathrm{C}(2 \mathrm{~A})-\mathrm{H}(2 \mathrm{~A} 2)$ & 117.8 \\
\hline $\mathrm{H}(2 \mathrm{~A} 1)-\mathrm{C}(2 \mathrm{~A})-\mathrm{H}(2 \mathrm{~A} 2)$ & 115.0 \\
\hline $\mathrm{N}(1 \mathrm{~A})-\mathrm{C}(3 \mathrm{~A})-\mathrm{C}(2 \mathrm{~A})$ & $59.8(3)$ \\
\hline$N(1 A)-C(3 A)-C(4 A)$ & $115.3(4)$ \\
\hline$C(2 A)-C(3 A)-C(4 A)$ & $126.6(4)$ \\
\hline $\mathrm{N}(1 \mathrm{~A})-\mathrm{C}(3 \mathrm{~A})-\mathrm{H}(3 \mathrm{~A} 1)$ & 114.4 \\
\hline $\mathrm{C}(2 \mathrm{~A})-\mathrm{C}(3 \mathrm{~A})-\mathrm{H}(3 \mathrm{~A} 1)$ & 114.4 \\
\hline $\mathrm{C}(4 \mathrm{~A})-\mathrm{C}(3 \mathrm{~A})-\mathrm{H}(3 \mathrm{~A} 1)$ & 114.4 \\
\hline $\mathrm{O}(1 \mathrm{~A})-\mathrm{C}(4 \mathrm{~A})-\mathrm{C}(12 \mathrm{~A})$ & $109.6(4)$ \\
\hline $\mathrm{O}(1 \mathrm{~A})-\mathrm{C}(4 \mathrm{~A})-\mathrm{C}(3 \mathrm{~A})$ & $105.5(3)$ \\
\hline$C(12 A)-C(4 A)-C(3 A)$ & $115.1(5)$ \\
\hline
\end{tabular}




$\begin{array}{ll}\text { O(1A)-C(4A)-H(4A) } & 108.8 \\ \text { C(12A)-C(4A)-H(4A) } & 108.8 \\ \text { C(3A)-C(4A)-H(4A) } & 108.8 \\ \text { C(6A)-C(5A)-C(10A) } & 119.7(4) \\ \text { C(6A)-C(5A)-S(1A) } & 120.7(3) \\ \text { C(10A)-C(5A)-S(1A) } & 119.5(3) \\ \text { C(7A)-C(6A)-C(5A) } & 120.0(4) \\ \text { C(7A)-C(6A)-H(6A) } & 120.0 \\ \text { C(5A)-C(6A)-H(6A) } & 120.0 \\ \text { C(6A)-C(7A)-C(8A) } & 120.7(4) \\ \text { C(6A)-C(7A)-H(7A) } & 119.6 \\ \text { C(8A)-C(7A)-H(7A) } & 119.6 \\ \text { C(7A)-C(8A)-C(9A) } & 118.9(4) \\ \text { C(7A)-C(8A)-C(11A) } & 121.4(4) \\ \text { C(9A)-C(8A)-C(11A) } & 119.7(4) \\ \text { C(10A)-C(9A)-C(8A) } & 120.9(4) \\ \text { C(10A)-C(9A)-H(9A) } & 119.5 \\ \text { C(8A)-C(9A)-H(9A) } & 119.5 \\ \text { C(9A)-C(10A)-C(5A) } & 119.6(4) \\ \text { C(9A)-C(10A)-H(10A) } & 120.2 \\ \text { C(5A)-C(10A)-H(10A) } & 120.2 \\ \text { C(8A)-C(11A)-H(11D) } & 109.5 \\ \text { C(8A)-C(11A)-H(11E) } & 109.5 \\ \text { H(11D)-C(11A)-H(11E) } & 109.5 \\ \text { C(8A)-C(11A)-H(11F) } & 109.5 \\ \text { H(11D)-C(11A)-H(11F) } & 109.5 \\ \text { H(11E)-C(11A)-H(11F) } & 109.5 \\ \text { C(4A)-C(12A)-H(12D) } & 109.5 \\ \text { C(4A)-C(12A)-H(12E) } & 109.5 \\ \text { H(12D)-C(12A)-H(12E) } & 109.5 \\ \text { C(4A)-C(12A)-H(12F) } & 109.5 \\ \text { H(12D)-C(12A)-H(12F) } & 109.5 \\ \end{array}$




\begin{tabular}{|c|c|}
\hline$C(15 A)-C(14 A)-C(13 A)$ & $121.3(4)$ \\
\hline $\mathrm{C}(15 \mathrm{~A})-\mathrm{C}(14 \mathrm{~A})-\mathrm{H}(14 \mathrm{~A})$ & 119.4 \\
\hline $\mathrm{C}(13 \mathrm{~A})-\mathrm{C}(14 \mathrm{~A})-\mathrm{H}(14 \mathrm{~A})$ & 119.4 \\
\hline $\mathrm{C}(14 \mathrm{~A})-\mathrm{C}(15 \mathrm{~A})-\mathrm{C}(16 \mathrm{~A})$ & $120.2(5)$ \\
\hline $\mathrm{C}(14 \mathrm{~A})-\mathrm{C}(15 \mathrm{~A})-\mathrm{H}(15 \mathrm{~A})$ & 119.9 \\
\hline $\mathrm{C}(16 \mathrm{~A})-\mathrm{C}(15 \mathrm{~A})-\mathrm{H}(15 \mathrm{~A})$ & 119.9 \\
\hline$C(17 A)-C(16 A)-C(15 A)$ & $119.2(5)$ \\
\hline $\mathrm{C}(17 \mathrm{~A})-\mathrm{C}(16 \mathrm{~A})-\mathrm{H}(16 \mathrm{~A})$ & 120.4 \\
\hline $\mathrm{C}(15 \mathrm{~A})-\mathrm{C}(16 \mathrm{~A})-\mathrm{H}(16 \mathrm{~A})$ & 120.4 \\
\hline$C(16 A)-C(17 A)-C(18 A)$ & $121.2(4)$ \\
\hline $\mathrm{C}(16 \mathrm{~A})-\mathrm{C}(17 \mathrm{~A})-\mathrm{H}(17 \mathrm{~A})$ & 119.4 \\
\hline $\mathrm{C}(18 \mathrm{~A})-\mathrm{C}(17 \mathrm{~A})-\mathrm{H}(17 \mathrm{~A})$ & 119.4 \\
\hline $\mathrm{C}(17 \mathrm{~A})-\mathrm{C}(18 \mathrm{~A})-\mathrm{C}(13 \mathrm{~A})$ & $120.5(5)$ \\
\hline $\mathrm{C}(17 \mathrm{~A})-\mathrm{C}(18 \mathrm{~A})-\mathrm{H}(18 \mathrm{~A})$ & 119.7 \\
\hline $\mathrm{C}(13 \mathrm{~A})-\mathrm{C}(18 \mathrm{~A})-\mathrm{H}(18 \mathrm{~A})$ & 119.7 \\
\hline$C(20 A)-C(19 A)-C(24 A)$ & $117.4(4)$ \\
\hline$C(20 A)-C(19 A)-C(1 A)$ & $121.3(4)$ \\
\hline$C(24 A)-C(19 A)-C(1 A)$ & $120.9(4)$ \\
\hline $\mathrm{C}(19 \mathrm{~A})-\mathrm{C}(20 \mathrm{~A})-\mathrm{C}(21 \mathrm{~A})$ & $122.0(5)$ \\
\hline $\mathrm{C}(19 \mathrm{~A})-\mathrm{C}(20 \mathrm{~A})-\mathrm{H}(20 \mathrm{~A})$ & 119.0 \\
\hline$C(21 A)-C(20 A)-H(20 A)$ & 119.0 \\
\hline $\mathrm{C}(22 \mathrm{~A})-\mathrm{C}(21 \mathrm{~A})-\mathrm{C}(20 \mathrm{~A})$ & $119.9(5)$ \\
\hline $\mathrm{C}(22 \mathrm{~A})-\mathrm{C}(21 \mathrm{~A})-\mathrm{H}(21 \mathrm{~A})$ & 120.1 \\
\hline $\mathrm{C}(20 \mathrm{~A})-\mathrm{C}(21 \mathrm{~A})-\mathrm{H}(21 \mathrm{~A})$ & 120.1 \\
\hline $\mathrm{C}(23 \mathrm{~A})-\mathrm{C}(22 \mathrm{~A})-\mathrm{C}(21 \mathrm{~A})$ & $119.1(5)$ \\
\hline $\mathrm{C}(23 \mathrm{~A})-\mathrm{C}(22 \mathrm{~A})-\mathrm{H}(22 \mathrm{~A})$ & 120.4 \\
\hline $\mathrm{C}(21 \mathrm{~A})-\mathrm{C}(22 \mathrm{~A})-\mathrm{H}(22 \mathrm{~A})$ & 120.4 \\
\hline $\mathrm{C}(22 \mathrm{~A})-\mathrm{C}(23 \mathrm{~A})-\mathrm{C}(24 \mathrm{~A})$ & $121.0(5)$ \\
\hline $\mathrm{C}(22 \mathrm{~A})-\mathrm{C}(23 \mathrm{~A})-\mathrm{H}(23 \mathrm{~A})$ & 119.5 \\
\hline $\mathrm{C}(24 \mathrm{~A})-\mathrm{C}(23 \mathrm{~A})-\mathrm{H}(23 \mathrm{~A})$ & 119.5 \\
\hline $\mathrm{C}(23 \mathrm{~A})-\mathrm{C}(24 \mathrm{~A})-\mathrm{C}(19 \mathrm{~A})$ & $120.6(5)$ \\
\hline $\mathrm{C}(23 \mathrm{~A})-\mathrm{C}(24 \mathrm{~A})-\mathrm{H}(24 \mathrm{~A})$ & 119.7 \\
\hline $\mathrm{C}(19 \mathrm{~A})-\mathrm{C}(24 \mathrm{~A})-\mathrm{H}(24 \mathrm{~A})$ & 119.7 \\
\hline$C(26 A)-C(25 A)-C(30 A)$ & $118.5(4)$ \\
\hline$C(26 A)-C(25 A)-C(1 A)$ & $120.6(4)$ \\
\hline$C(30 A)-C(25 A)-C(1 A)$ & $120.7(4)$ \\
\hline
\end{tabular}




$\begin{array}{ll}\mathrm{C}(25 \mathrm{~A})-\mathrm{C}(26 \mathrm{~A})-\mathrm{C}(27 \mathrm{~A}) & 119.8(4) \\ \mathrm{C}(25 \mathrm{~A})-\mathrm{C}(26 \mathrm{~A})-\mathrm{H}(26 \mathrm{~A}) & 120.1 \\ \mathrm{C}(27 \mathrm{~A})-\mathrm{C}(26 \mathrm{~A})-\mathrm{H}(26 \mathrm{~A}) & 120.1 \\ \mathrm{C}(28 \mathrm{~A})-\mathrm{C}(27 \mathrm{~A})-\mathrm{C}(26 \mathrm{~A}) & 120.9(5) \\ \mathrm{C}(28 \mathrm{~A})-\mathrm{C}(27 \mathrm{~A})-\mathrm{H}(27 \mathrm{~A}) & 119.5 \\ \mathrm{C}(26 \mathrm{~A})-\mathrm{C}(27 \mathrm{~A})-\mathrm{H}(27 \mathrm{~A}) & 119.5 \\ \mathrm{C}(29 \mathrm{~A})-\mathrm{C}(28 \mathrm{~A})-\mathrm{C}(27 \mathrm{~A}) & 119.8(5) \\ \mathrm{C}(29 \mathrm{~A})-\mathrm{C}(28 \mathrm{~A})-\mathrm{H}(28 \mathrm{~A}) & 120.1 \\ \mathrm{C}(27 \mathrm{~A})-\mathrm{C}(28 \mathrm{~A})-\mathrm{H}(28 \mathrm{~A}) & 120.1 \\ \mathrm{C}(28 \mathrm{~A})-\mathrm{C}(29 \mathrm{~A})-\mathrm{C}(30 \mathrm{~A}) & 120.1(5) \\ \mathrm{C}(28 \mathrm{~A})-\mathrm{C}(29 \mathrm{~A})-\mathrm{H}(29 \mathrm{~A}) & 120.0 \\ \mathrm{C}(30 \mathrm{~A})-\mathrm{C}(29 \mathrm{~A})-\mathrm{H}(29 \mathrm{~A}) & 120.0 \\ \mathrm{C}(29 \mathrm{~A})-\mathrm{C}(30 \mathrm{~A})-\mathrm{C}(25 \mathrm{~A}) & 120.8(4) \\ \mathrm{C}(29 \mathrm{~A})-\mathrm{C}(30 \mathrm{~A})-\mathrm{H}(30 \mathrm{~A}) & 119.6 \\ \mathrm{C}(25 \mathrm{~A})-\mathrm{C}(30 \mathrm{~A})-\mathrm{H}(30 \mathrm{~A}) & 119.6\end{array}$

Symmetry transformations used to generate equivalent atoms: 
Table 4. Anisotropic displacement parameters $\left(\AA^{2} \times 10^{3}\right)$ for 25. The anisotropic displacement factor exponent takes the form: $-2 \pi^{2}\left[h^{2} a^{* 2} U^{11}+\ldots+2 h k a^{*} b * U^{12}\right]$

\begin{tabular}{|c|c|c|c|c|c|c|}
\hline & $\mathrm{U}^{11}$ & $\mathrm{U}^{22}$ & $\mathrm{U}^{33}$ & $\mathrm{U}^{23}$ & $\mathrm{U}^{13}$ & $\mathrm{U}^{12}$ \\
\hline$S(1)$ & $35(1)$ & $37(1)$ & $34(1)$ & $4(1)$ & $13(1)$ & $0(1)$ \\
\hline $\mathrm{N}(1)$ & $34(2)$ & $27(2)$ & $26(2)$ & $1(2)$ & $10(2)$ & $-1(2)$ \\
\hline $\mathrm{O}(1)$ & $36(2)$ & $31(2)$ & $35(2)$ & $6(2)$ & $12(2)$ & $2(2)$ \\
\hline $\mathrm{O}(2)$ & $43(2)$ & $50(2)$ & $28(2)$ & $16(2)$ & $5(2)$ & $10(2)$ \\
\hline $\mathrm{O}(3)$ & $47(2)$ & $56(3)$ & $51(2)$ & $-3(2)$ & $27(2)$ & $-13(2)$ \\
\hline$C(1)$ & $28(2)$ & $26(3)$ & $26(3)$ & $1(2)$ & $9(2)$ & $-2(2)$ \\
\hline$C(2)$ & $32(2)$ & $31(3)$ & $30(3)$ & $5(2)$ & $10(2)$ & $1(2)$ \\
\hline$C(3)$ & $67(4)$ & $35(3)$ & $33(3)$ & $6(2)$ & $13(3)$ & $18(3)$ \\
\hline$C(4)$ & $24(2)$ & 41(3) & $25(3)$ & $8(2)$ & $7(2)$ & $-5(2)$ \\
\hline$C(5)$ & $22(2)$ & $28(3)$ & $35(3)$ & $1(2)$ & $5(2)$ & $0(2)$ \\
\hline$C(6)$ & $26(3)$ & $53(4)$ & $43(3)$ & $-3(3)$ & $6(2)$ & $7(2)$ \\
\hline$C(7)$ & $30(3)$ & $49(4)$ & $35(3)$ & $-3(3)$ & $-1(2)$ & $0(2)$ \\
\hline$C(8)$ & $30(3)$ & $23(3)$ & $32(3)$ & $5(2)$ & $7(2)$ & $-8(2)$ \\
\hline$C(9)$ & $24(3)$ & $58(4)$ & $41(3)$ & 1(3) & $4(2)$ & $13(2)$ \\
\hline$C(10)$ & $28(3)$ & $57(4)$ & $38(3)$ & $-2(3)$ & $-2(2)$ & $10(3)$ \\
\hline$C(11)$ & $38(3)$ & $37(3)$ & $33(3)$ & $5(2)$ & $7(2)$ & $5(2)$ \\
\hline$C(12)$ & $58(3)$ & $54(4)$ & $25(3)$ & $4(3)$ & $6(2)$ & $-4(3)$ \\
\hline$C(13)$ & $27(3)$ & $27(3)$ & $33(3)$ & $0(2)$ & $8(2)$ & $-5(2)$ \\
\hline$C(14)$ & $32(3)$ & $33(3)$ & $53(3)$ & $3(3)$ & $16(2)$ & $1(2)$ \\
\hline$C(15)$ & $46(3)$ & 41(3) & $65(4)$ & $7(3)$ & $19(3)$ & $14(3)$ \\
\hline$C(16)$ & $46(3)$ & $33(3)$ & $67(4)$ & $-5(3)$ & $29(3)$ & 1(3) \\
\hline $\mathrm{C}(17)$ & $51(3)$ & $39(3)$ & $54(4)$ & $-7(3)$ & $27(3)$ & $-1(3)$ \\
\hline $\mathrm{C}(18)$ & $34(3)$ & $39(3)$ & $34(3)$ & $0(2)$ & $17(2)$ & $-2(2)$ \\
\hline$C(19)$ & $29(2)$ & $35(3)$ & $25(3)$ & $5(2)$ & $13(2)$ & $2(2)$ \\
\hline $\mathrm{C}(20)$ & $31(3)$ & $55(4)$ & $30(3)$ & $-1(3)$ & $11(2)$ & $-11(3)$ \\
\hline $\mathrm{C}(21)$ & $35(3)$ & $76(5)$ & $31(3)$ & $-7(3)$ & $11(2)$ & $-19(3)$ \\
\hline$C(22)$ & $25(3)$ & $92(5)$ & $41(3)$ & $-2(3)$ & $10(2)$ & $-13(3)$ \\
\hline $\mathrm{C}(23)$ & $37(3)$ & 71(4) & $36(3)$ & $4(3)$ & $8(2)$ & $10(3)$ \\
\hline$C(24)$ & $38(3)$ & $45(3)$ & $25(3)$ & $3(2)$ & $11(2)$ & $4(3)$ \\
\hline$C(25)$ & $26(2)$ & $25(3)$ & $20(2)$ & $0(2)$ & $6(2)$ & $0(2)$ \\
\hline$C(26)$ & $32(3)$ & $43(3)$ & $23(3)$ & $-2(2)$ & $3(2)$ & $-4(2)$ \\
\hline
\end{tabular}




\begin{tabular}{|c|c|c|c|c|c|c|}
\hline $\mathrm{C}(27)$ & $37(3)$ & $41(3)$ & $26(3)$ & $0(2)$ & $11(2)$ & $-12(2)$ \\
\hline$C(28)$ & $40(3)$ & $30(3)$ & $28(3)$ & $3(2)$ & $12(2)$ & $-1(2)$ \\
\hline$C(29)$ & $37(3)$ & $35(3)$ & $30(3)$ & $-2(2)$ & $4(2)$ & $-13(2)$ \\
\hline $\mathrm{C}(30)$ & $39(3)$ & $37(3)$ & $25(3)$ & $0(2)$ & $8(2)$ & $-4(2)$ \\
\hline $\mathrm{S}(1 \mathrm{~A})$ & $39(1)$ & $40(1)$ & $27(1)$ & $3(1)$ & $4(1)$ & $5(1)$ \\
\hline $\mathrm{N}(1 \mathrm{~A})$ & $30(2)$ & $30(2)$ & $27(2)$ & $0(2)$ & $2(2)$ & $1(2)$ \\
\hline $\mathrm{O}(1 \mathrm{~A})$ & $42(2)$ & $29(2)$ & $35(2)$ & $2(2)$ & $0(2)$ & $7(2)$ \\
\hline $\mathrm{O}(2 \mathrm{~A})$ & $67(2)$ & $43(2)$ & $43(2)$ & $9(2)$ & $28(2)$ & $-10(2)$ \\
\hline $\mathrm{O}(3 \mathrm{~A})$ & $52(2)$ & 59(3) & $38(2)$ & $4(2)$ & $-12(2)$ & $19(2)$ \\
\hline $\mathrm{C}(1 \mathrm{~A})$ & $30(2)$ & $28(3)$ & $26(3)$ & $-2(2)$ & $6(2)$ & $0(2)$ \\
\hline $\mathrm{C}(2 \mathrm{~A})$ & $47(3)$ & $37(3)$ & $25(3)$ & $-1(2)$ & $-4(2)$ & $-11(2)$ \\
\hline $\mathrm{C}(3 \mathrm{~A})$ & $31(3)$ & $33(3)$ & $24(3)$ & $5(2)$ & $-2(2)$ & $4(2)$ \\
\hline $\mathrm{C}(4 \mathrm{~A})$ & 29(3) & $60(4)$ & 21(3) & $9(2)$ & $6(2)$ & $12(3)$ \\
\hline $\mathrm{C}(5 \mathrm{~A})$ & 29(3) & $36(3)$ & $25(3)$ & $-2(2)$ & $4(2)$ & $-4(2)$ \\
\hline$C(6 A)$ & $24(3)$ & $47(3)$ & $32(3)$ & $4(2)$ & $-2(2)$ & $-1(2)$ \\
\hline$C(7 A)$ & $25(3)$ & $45(3)$ & $36(3)$ & $8(3)$ & $14(2)$ & $4(2)$ \\
\hline $\mathrm{C}(8 \mathrm{~A})$ & $32(3)$ & 29(3) & $28(3)$ & $7(2)$ & $12(2)$ & $-4(2)$ \\
\hline $\mathrm{C}(9 \mathrm{~A})$ & $32(3)$ & $38(3)$ & $28(3)$ & $-1(2)$ & $0(2)$ & $4(2)$ \\
\hline$C(10 A)$ & $27(3)$ & $33(3)$ & $33(3)$ & $1(2)$ & $9(2)$ & $0(2)$ \\
\hline $\mathrm{C}(11 \mathrm{~A})$ & $43(3)$ & $51(4)$ & $23(3)$ & $-1(2)$ & $13(2)$ & $1(3)$ \\
\hline $\mathrm{C}(12 \mathrm{~A})$ & $51(3)$ & $54(4)$ & 29(3) & $-4(3)$ & $13(2)$ & $2(3)$ \\
\hline$C(13 A)$ & $26(2)$ & $33(3)$ & $26(3)$ & $1(2)$ & $7(2)$ & $1(2)$ \\
\hline $\mathrm{C}(14 \mathrm{~A})$ & $37(3)$ & $39(3)$ & $26(3)$ & $4(2)$ & $11(2)$ & $9(2)$ \\
\hline$C(15 A)$ & $43(3)$ & $38(3)$ & $31(3)$ & $5(2)$ & $1(2)$ & $9(2)$ \\
\hline$C(16 A)$ & $38(3)$ & $32(3)$ & $43(3)$ & $9(3)$ & $7(2)$ & $7(2)$ \\
\hline $\mathrm{C}(17 \mathrm{~A})$ & $36(3)$ & $34(3)$ & $32(3)$ & $-4(2)$ & $11(2)$ & $2(2)$ \\
\hline $\mathrm{C}(18 \mathrm{~A})$ & $31(3)$ & 44(3) & 28(3) & $-2(2)$ & $7(2)$ & $3(2)$ \\
\hline $\mathrm{C}(19 \mathrm{~A})$ & $26(2)$ & $37(3)$ & $25(3)$ & $5(2)$ & $3(2)$ & $0(2)$ \\
\hline$C(20 A)$ & $36(3)$ & $43(3)$ & $33(3)$ & $-2(3)$ & $12(2)$ & $-5(3)$ \\
\hline$C(21 \mathrm{~A})$ & $37(3)$ & $56(4)$ & $46(3)$ & $4(3)$ & $15(3)$ & $-9(3)$ \\
\hline $\mathrm{C}(22 \mathrm{~A})$ & $34(3)$ & $76(5)$ & $48(4)$ & $2(3)$ & $17(3)$ & $4(3)$ \\
\hline$C(23 \mathrm{~A})$ & $37(3)$ & $54(4)$ & $37(3)$ & $-8(3)$ & $10(2)$ & $12(3)$ \\
\hline $\mathrm{C}(24 \mathrm{~A})$ & $39(3)$ & $39(3)$ & $26(3)$ & $4(2)$ & $8(2)$ & $10(2)$ \\
\hline$C(25 \mathrm{~A})$ & $26(2)$ & $25(3)$ & $27(3)$ & $1(2)$ & $3(2)$ & $5(2)$ \\
\hline$C(26 \mathrm{~A})$ & $32(3)$ & $30(3)$ & $27(3)$ & $-1(2)$ & $5(2)$ & $0(2)$ \\
\hline $\mathrm{C}(27 \mathrm{~A})$ & $43(3)$ & $32(3)$ & $34(3)$ & $0(2)$ & $-3(2)$ & $7(2)$ \\
\hline
\end{tabular}




\begin{tabular}{lllllll}
$\mathrm{C}(28 \mathrm{~A})$ & $34(3)$ & $30(3)$ & $40(3)$ & $-7(2)$ & $2(2)$ & $-1(2)$ \\
$\mathrm{C}(29 \mathrm{~A})$ & $28(3)$ & $41(3)$ & $38(3)$ & $-1(2)$ & $9(2)$ & $-2(2)$ \\
$\mathrm{C}(30 \mathrm{~A})$ & $30(3)$ & $35(3)$ & $24(3)$ & $0(2)$ & $7(2)$ & $4(2)$ \\
\hline
\end{tabular}


Table 5. Hydrogen coordinates ( $\left.\times 10^{4}\right)$ and isotropic displacement parameters $\left(\AA^{2} \times 10^{3}\right)$ for 25 .

\begin{tabular}{|c|c|c|c|c|}
\hline & $\mathrm{x}$ & $\mathrm{y}$ & $\mathrm{z}$ & $\mathrm{U}(\mathrm{eq})$ \\
\hline $\mathrm{H}(2)$ & 10350 & 5701 & 9177 & 37 \\
\hline $\mathrm{H}(3 \mathrm{~A})$ & 8965 & 4508 & 8183 & 53 \\
\hline $\mathrm{H}(3 \mathrm{~B})$ & 10091 & 4498 & 9210 & 53 \\
\hline $\mathrm{H}(4)$ & 8238 & 6284 & 8015 & 35 \\
\hline $\mathrm{H}(6)$ & 12214 & 7358 & 9299 & 49 \\
\hline $\mathrm{H}(7)$ & 11840 & 7770 & 10739 & 46 \\
\hline $\mathrm{H}(9)$ & 7371 & 7953 & 9803 & 49 \\
\hline $\mathrm{H}(10)$ & 7747 & 7586 & 8350 & 50 \\
\hline $\mathrm{H}(11 \mathrm{~A})$ & 10242 & 8231 & 11757 & 54 \\
\hline $\mathrm{H}(11 \mathrm{~B})$ & 8779 & 7752 & 11662 & 54 \\
\hline $\mathrm{H}(11 \mathrm{C})$ & 8627 & 8522 & 11300 & 54 \\
\hline $\mathrm{H}(12 \mathrm{~A})$ & 8077 & 5882 & 6485 & 69 \\
\hline $\mathrm{H}(12 \mathrm{~B})$ & 7492 & 5293 & 7101 & 69 \\
\hline $\mathrm{H}(12 \mathrm{C})$ & 9153 & 5251 & 6824 & 69 \\
\hline $\mathrm{H}(14)$ & 6254 & 5955 & 9043 & 46 \\
\hline $\mathrm{H}(15)$ & 4648 & 6844 & 9358 & 59 \\
\hline $\mathrm{H}(16)$ & 4799 & 7278 & 10850 & 56 \\
\hline $\mathrm{H}(17)$ & 6531 & 6821 & 12050 & 55 \\
\hline $\mathrm{H}(18)$ & 8123 & 5939 & 11758 & 41 \\
\hline $\mathrm{H}(20)$ & 10174 & 6299 & 10731 & 46 \\
\hline $\mathrm{H}(21)$ & 12755 & 6344 & 11304 & 56 \\
\hline $\mathrm{H}(22)$ & 14145 & 5339 & 11613 & 63 \\
\hline $\mathrm{H}(23)$ & 12918 & 4289 & 11370 & 57 \\
\hline $\mathrm{H}(24)$ & 10339 & 4235 & 10812 & 43 \\
\hline $\mathrm{H}(26)$ & 8222 & 4677 & 11867 & 39 \\
\hline $\mathrm{H}(27)$ & 6794 & 3784 & 12309 & 41 \\
\hline $\mathrm{H}(28)$ & 5162 & 3176 & 11215 & 38 \\
\hline $\mathrm{H}(29)$ & 4899 & 3491 & 9652 & 41 \\
\hline $\mathrm{H}(30)$ & 6341 & 4387 & 9185 & 40 \\
\hline $\mathrm{H}(2 \mathrm{~A} 1)$ & 4005 & 6093 & 6946 & 44 \\
\hline
\end{tabular}




\begin{tabular}{|c|c|c|c|c|}
\hline $\mathrm{H}(2 \mathrm{~A} 2)$ & 4859 & 6124 & 6005 & 44 \\
\hline $\mathrm{H}(3 \mathrm{~A} 1)$ & 4907 & 4924 & 5922 & 36 \\
\hline $\mathrm{H}(4 \mathrm{~A})$ & 3041 & 4309 & 6793 & 44 \\
\hline $\mathrm{H}(6 \mathrm{~A})$ & 7371 & 3147 & 6179 & 42 \\
\hline $\mathrm{H}(7 \mathrm{~A})$ & 7222 & 2840 & 4625 & 41 \\
\hline $\mathrm{H}(9 \mathrm{~A})$ & 2669 & 2983 & 4304 & 39 \\
\hline $\mathrm{H}(10 \mathrm{~A})$ & 2796 & 3255 & 5866 & 37 \\
\hline $\mathrm{H}(11 \mathrm{D})$ & 4058 & 2380 & 3125 & 58 \\
\hline $\mathrm{H}(11 \mathrm{E})$ & 5807 & 2568 & 3160 & 58 \\
\hline $\mathrm{H}(11 \mathrm{~F})$ & 4540 & 3144 & 2920 & 58 \\
\hline $\mathrm{H}(12 \mathrm{D})$ & 3010 & 4623 & 8319 & 66 \\
\hline $\mathrm{H}(12 \mathrm{E})$ & 2467 & 5273 & 7694 & 66 \\
\hline $\mathrm{H}(12 \mathrm{~F})$ & 4157 & 5239 & 8245 & 66 \\
\hline $\mathrm{H}(14 \mathrm{~A})$ & 1982 & 6035 & 3099 & 40 \\
\hline $\mathrm{H}(15 \mathrm{~A})$ & 375 & 6915 & 2538 & 45 \\
\hline $\mathrm{H}(16 \mathrm{~A})$ & -827 & 7562 & 3557 & 45 \\
\hline $\mathrm{H}(17 \mathrm{~A})$ & -502 & 7279 & 5120 & 40 \\
\hline $\mathrm{H}(18 \mathrm{~A})$ & 1046 & 6380 & 5693 & 41 \\
\hline $\mathrm{H}(20 \mathrm{~A})$ & 4412 & 6527 & 4370 & 44 \\
\hline $\mathrm{H}(21 \mathrm{~A})$ & 6802 & 6555 & 3927 & 54 \\
\hline $\mathrm{H}(22 \mathrm{~A})$ & 8069 & 5535 & 3715 & 62 \\
\hline $\mathrm{H}(23 \mathrm{~A})$ & 6880 & 4500 & 3903 & 51 \\
\hline $\mathrm{H}(24 \mathrm{~A})$ & 4496 & 4469 & 4350 & 42 \\
\hline $\mathrm{H}(26 \mathrm{~A})$ & 2141 & 4797 & 3161 & 36 \\
\hline $\mathrm{H}(27 \mathrm{~A})$ & 540 & 3874 & 2708 & 45 \\
\hline $\mathrm{H}(28 \mathrm{~A})$ & -852 & 3375 & 3745 & 42 \\
\hline $\mathrm{H}(29 \mathrm{~A})$ & -707 & 3807 & 5244 & 42 \\
\hline $\mathrm{H}(30 \mathrm{~A})$ & 878 & 4724 & 5716 & 35 \\
\hline
\end{tabular}




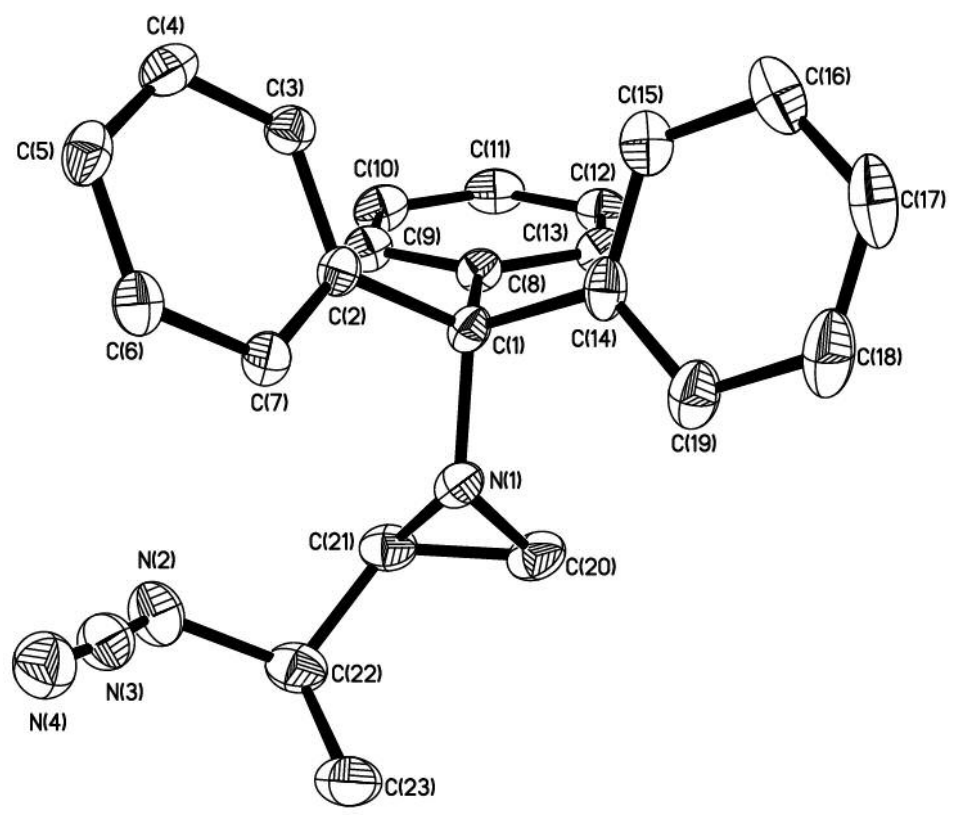

ORTEP of compound $\mathbf{2 6}$ 
Table 1. Crystal data and structure refinement for $\mathbf{2 6}$.

Identification code

ohj135p61

Empirical formula

C23 H22 N4

Formula weight

354.45

Temperature

150(2) K

Wavelength

$0.71073 \AA$

Crystal system

Hexagonal

Space group

Unit cell dimensions

$\mathrm{P}_{1}$

$\mathrm{a}=8.9977(4) \AA \quad \alpha=90^{\circ}$.

$\mathrm{b}=8.9977(4) \AA \quad \beta=90^{\circ}$.

$\mathrm{c}=40.312(2) \AA \quad \gamma=120^{\circ}$.

Volume

2826.4(2) $\AA^{3}$

$\mathrm{Z}$

6

Density (calculated)

$1.249 \mathrm{Mg} / \mathrm{m}^{3}$

Absorption coefficient

$0.076 \mathrm{~mm}^{-1}$

$\mathrm{F}(000)$

1128

Crystal size

$0.34 \times 0.21 \times 0.21 \mathrm{~mm}^{3}$

Theta range for data collection

2.61 to $30.68^{\circ}$.

Index ranges

$-12<=\mathrm{h}<=12,-12<=\mathrm{k}<=12,-57<=\mathrm{l}<=57$

Reflections collected

59143

Independent reflections

$2932[\mathrm{R}(\mathrm{int})=0.0648]$

Completeness to theta $=25.00^{\circ}$

Absorption correction

$100.0 \%$

Semi-empirical from equivalents

Max. and min. transmission

0.9843 and 0.9747

Refinement method

Full-matrix least-squares on $\mathrm{F}^{2}$

Data / restraints / parameters

2932 / 1 / 245

Goodness-of-fit on $\mathrm{F}^{2}$

1.132

Final R indices [I $>2 \operatorname{sigma}(\mathrm{I})]$

$\mathrm{R} 1=0.0443, \mathrm{wR} 2=0.1171$

$\mathrm{R}$ indices (all data)

$\mathrm{R} 1=0.0497, \mathrm{wR} 2=0.1203$

Absolute structure parameter

10(10)

Largest diff. peak and hole

0.367 and -0.170 e. $\AA^{-3}$ 
Table 2. Atomic coordinates ( $\left.\mathrm{x} 10^{4}\right)$ and equivalent isotropic displacement parameters $\left(\AA^{2} \mathrm{x} 10^{3}\right)$ for 26. $U(e q)$ is defined as one third of the trace of the orthogonalized $U^{i j}$ tensor.

\begin{tabular}{|c|c|c|c|c|}
\hline & $\mathrm{x}$ & $\mathrm{y}$ & $\mathrm{z}$ & $\mathrm{U}(\mathrm{eq})$ \\
\hline $\mathrm{N}(1)$ & $7443(2)$ & $196(2)$ & $8283(1)$ & $23(1)$ \\
\hline $\mathrm{N}(2)$ & $3735(3)$ & $-2099(3)$ & $7936(1)$ & $35(1)$ \\
\hline $\mathrm{N}(3)$ & $3311(3)$ & $-3535(3)$ & $8044(1)$ & $32(1)$ \\
\hline $\mathrm{N}(4)$ & 2792(3) & $-4885(3)$ & $8148(1)$ & $37(1)$ \\
\hline $\mathrm{C}(1)$ & $7480(2)$ & 1011(2) & $8610(1)$ & 19(1) \\
\hline$C(2)$ & $5893(2)$ & $-294(2)$ & $8808(1)$ & 19(1) \\
\hline$C(3)$ & $5220(3)$ & $213(3)$ & $9072(1)$ & $22(1)$ \\
\hline $\mathrm{C}(4)$ & $3853(3)$ & $-997(3)$ & $9259(1)$ & $27(1)$ \\
\hline$C(5)$ & $3126(3)$ & $-2741(3)$ & 9191(1) & $26(1)$ \\
\hline$C(6)$ & $3789(3)$ & $-3265(3)$ & $8934(1)$ & $25(1)$ \\
\hline$C(7)$ & $5163(3)$ & $-2050(2)$ & $8745(1)$ & $22(1)$ \\
\hline $\mathrm{C}(8)$ & $7579(2)$ & $2763(2)$ & $8569(1)$ & $20(1)$ \\
\hline$C(9)$ & 6092(3) & 2861(3) & $8518(1)$ & $24(1)$ \\
\hline$C(10)$ & 6191(3) & 4442(3) & $8464(1)$ & $29(1)$ \\
\hline $\mathrm{C}(11)$ & $7767(3)$ & 5933(3) & $8457(1)$ & $30(1)$ \\
\hline$C(12)$ & $9260(3)$ & $5860(3)$ & $8501(1)$ & $27(1)$ \\
\hline $\mathrm{C}(13)$ & $9165(3)$ & $4288(2)$ & $8557(1)$ & $23(1)$ \\
\hline$C(14)$ & $9070(2)$ & $1243(2)$ & $8802(1)$ & $24(1)$ \\
\hline $\mathrm{C}(15)$ & $9467(3)$ & 2060(3) & $9109(1)$ & $28(1)$ \\
\hline$C(16)$ & 10881(3) & 2283(3) & $9290(1)$ & $34(1)$ \\
\hline$C(17)$ & $11908(3)$ & 1660(3) & $9165(1)$ & $39(1)$ \\
\hline $\mathrm{C}(18)$ & 11514(3) & $827(3)$ & $8863(1)$ & $40(1)$ \\
\hline$C(19)$ & $10115(3)$ & $626(3)$ & $8679(1)$ & $31(1)$ \\
\hline$C(20)$ & $8480(3)$ & 1281(3) & $8006(1)$ & $31(1)$ \\
\hline$C(21)$ & $6575(3)$ & $426(3)$ & 7995(1) & $27(1)$ \\
\hline$C(22)$ & 5491(3) & $-1116(3)$ & $7777(1)$ & $31(1)$ \\
\hline$C(23)$ & $6261(4)$ & $-2236(3)$ & 7699(1) & $36(1)$ \\
\hline
\end{tabular}


Table 3. Bond lengths [ $\left[\AA\right.$ ] and angles $\left[{ }^{\circ}\right]$ for 26.

\begin{tabular}{|c|c|}
\hline $\mathrm{N}(1)-\mathrm{C}(20)$ & $1.471(3)$ \\
\hline $\mathrm{N}(1)-\mathrm{C}(21)$ & $1.471(3)$ \\
\hline $\mathrm{N}(1)-\mathrm{C}(1)$ & $1.500(3)$ \\
\hline $\mathrm{N}(2)-\mathrm{N}(3)$ & $1.230(3)$ \\
\hline $\mathrm{N}(2)-\mathrm{C}(22)$ & $1.512(3)$ \\
\hline $\mathrm{N}(3)-\mathrm{N}(4)$ & $1.141(3)$ \\
\hline$C(1)-C(2)$ & $1.542(3)$ \\
\hline$C(1)-C(8)$ & $1.542(3)$ \\
\hline $\mathrm{C}(1)-\mathrm{C}(14)$ & $1.547(3)$ \\
\hline$C(2)-C(7)$ & $1.398(3)$ \\
\hline$C(2)-C(3)$ & $1.407(3)$ \\
\hline$C(3)-C(4)$ & $1.389(3)$ \\
\hline $\mathrm{C}(3)-\mathrm{H}(3)$ & 0.9500 \\
\hline$C(4)-C(5)$ & $1.392(3)$ \\
\hline $\mathrm{C}(4)-\mathrm{H}(4)$ & 0.9500 \\
\hline$C(5)-C(6)$ & $1.391(3)$ \\
\hline $\mathrm{C}(5)-\mathrm{H}(5)$ & 0.9500 \\
\hline$C(6)-C(7)$ & $1.396(3)$ \\
\hline $\mathrm{C}(6)-\mathrm{H}(6)$ & 0.9500 \\
\hline $\mathrm{C}(7)-\mathrm{H}(7)$ & 0.9500 \\
\hline $\mathrm{C}(8)-\mathrm{C}(9)$ & $1.399(3)$ \\
\hline$C(8)-C(13)$ & $1.401(3)$ \\
\hline C(9)-C(10) & $1.397(3)$ \\
\hline $\mathrm{C}(9)-\mathrm{H}(9)$ & 0.9500 \\
\hline$C(10)-C(11)$ & $1.382(4)$ \\
\hline $\mathrm{C}(10)-\mathrm{H}(10)$ & 0.9500 \\
\hline $\mathrm{C}(11)-\mathrm{C}(12)$ & $1.389(3)$ \\
\hline $\mathrm{C}(11)-\mathrm{H}(11)$ & 0.9500 \\
\hline$C(12)-C(13)$ & $1.392(3)$ \\
\hline $\mathrm{C}(12)-\mathrm{H}(12)$ & 0.9500 \\
\hline $\mathrm{C}(13)-\mathrm{H}(13)$ & 0.9500 \\
\hline$C(14)-C(15)$ & $1.390(3)$ \\
\hline $\mathrm{C}(14)-\mathrm{C}(19)$ & $1.399(3)$ \\
\hline$C(15)-C(16)$ & $1.392(3)$ \\
\hline
\end{tabular}




\begin{tabular}{|c|c|}
\hline $\mathrm{C}(15)-\mathrm{H}(15)$ & 0.9500 \\
\hline$C(16)-C(17)$ & $1.393(4)$ \\
\hline $\mathrm{C}(16)-\mathrm{H}(16)$ & 0.9500 \\
\hline $\mathrm{C}(17)-\mathrm{C}(18)$ & $1.378(4)$ \\
\hline $\mathrm{C}(17)-\mathrm{H}(17)$ & 0.9500 \\
\hline $\mathrm{C}(18)-\mathrm{C}(19)$ & $1.394(3)$ \\
\hline $\mathrm{C}(18)-\mathrm{H}(18)$ & 0.9500 \\
\hline $\mathrm{C}(19)-\mathrm{H}(19)$ & 0.9500 \\
\hline$C(20)-C(21)$ & $1.488(3)$ \\
\hline $\mathrm{C}(20)-\mathrm{H}(20 \mathrm{~A})$ & 0.9900 \\
\hline $\mathrm{C}(20)-\mathrm{H}(20 \mathrm{~B})$ & 0.9900 \\
\hline $\mathrm{C}(21)-\mathrm{C}(22)$ & $1.514(3)$ \\
\hline $\mathrm{C}(21)-\mathrm{H}(21)$ & 1.0000 \\
\hline $\mathrm{C}(22)-\mathrm{C}(23)$ & $1.514(4)$ \\
\hline $\mathrm{C}(22)-\mathrm{H}(22)$ & 1.0000 \\
\hline $\mathrm{C}(23)-\mathrm{H}(23 \mathrm{~A})$ & 0.9800 \\
\hline $\mathrm{C}(23)-\mathrm{H}(23 \mathrm{~B})$ & 0.9800 \\
\hline $\mathrm{C}(23)-\mathrm{H}(23 \mathrm{C})$ & 0.9800 \\
\hline $\mathrm{C}(20)-\mathrm{N}(1)-\mathrm{C}(21)$ & $60.77(15)$ \\
\hline $\mathrm{C}(20)-\mathrm{N}(1)-\mathrm{C}(1)$ & $119.42(16)$ \\
\hline $\mathrm{C}(21)-\mathrm{N}(1)-\mathrm{C}(1)$ & $120.53(15)$ \\
\hline $\mathrm{N}(3)-\mathrm{N}(2)-\mathrm{C}(22)$ & $116.7(2)$ \\
\hline $\mathrm{N}(4)-\mathrm{N}(3)-\mathrm{N}(2)$ & $174.6(3)$ \\
\hline $\mathrm{N}(1)-\mathrm{C}(1)-\mathrm{C}(2)$ & $107.27(15)$ \\
\hline $\mathrm{N}(1)-\mathrm{C}(1)-\mathrm{C}(8)$ & $112.44(16)$ \\
\hline $\mathrm{C}(2)-\mathrm{C}(1)-\mathrm{C}(8)$ & $113.08(15)$ \\
\hline $\mathrm{N}(1)-\mathrm{C}(1)-\mathrm{C}(14)$ & $107.33(15)$ \\
\hline $\mathrm{C}(2)-\mathrm{C}(1)-\mathrm{C}(14)$ & $106.59(16)$ \\
\hline $\mathrm{C}(8)-\mathrm{C}(1)-\mathrm{C}(14)$ & $109.80(15)$ \\
\hline $\mathrm{C}(7)-\mathrm{C}(2)-\mathrm{C}(3)$ & $117.90(17)$ \\
\hline $\mathrm{C}(7)-\mathrm{C}(2)-\mathrm{C}(1)$ & $119.98(17)$ \\
\hline $\mathrm{C}(3)-\mathrm{C}(2)-\mathrm{C}(1)$ & 121.94(17) \\
\hline $\mathrm{C}(4)-\mathrm{C}(3)-\mathrm{C}(2)$ & $120.83(19)$ \\
\hline $\mathrm{C}(4)-\mathrm{C}(3)-\mathrm{H}(3)$ & 119.6 \\
\hline $\mathrm{C}(2)-\mathrm{C}(3)-\mathrm{H}(3)$ & 119.6 \\
\hline
\end{tabular}




\begin{tabular}{|c|c|}
\hline$C(3)-C(4)-C(5)$ & $120.62(19)$ \\
\hline $\mathrm{C}(3)-\mathrm{C}(4)-\mathrm{H}(4)$ & 119.7 \\
\hline $\mathrm{C}(5)-\mathrm{C}(4)-\mathrm{H}(4)$ & 119.7 \\
\hline$C(6)-C(5)-C(4)$ & $119.30(19)$ \\
\hline $\mathrm{C}(6)-\mathrm{C}(5)-\mathrm{H}(5)$ & 120.3 \\
\hline $\mathrm{C}(4)-\mathrm{C}(5)-\mathrm{H}(5)$ & 120.3 \\
\hline$C(5)-C(6)-C(7)$ & $120.2(2)$ \\
\hline $\mathrm{C}(5)-\mathrm{C}(6)-\mathrm{H}(6)$ & 119.9 \\
\hline$C(7)-C(6)-H(6)$ & 119.9 \\
\hline$C(6)-C(7)-C(2)$ & $121.19(19)$ \\
\hline $\mathrm{C}(6)-\mathrm{C}(7)-\mathrm{H}(7)$ & 119.4 \\
\hline $\mathrm{C}(2)-\mathrm{C}(7)-\mathrm{H}(7)$ & 119.4 \\
\hline $\mathrm{C}(9)-\mathrm{C}(8)-\mathrm{C}(13)$ & $118.10(18)$ \\
\hline $\mathrm{C}(9)-\mathrm{C}(8)-\mathrm{C}(1)$ & $120.76(17)$ \\
\hline $\mathrm{C}(13)-\mathrm{C}(8)-\mathrm{C}(1)$ & $120.97(17)$ \\
\hline $\mathrm{C}(10)-\mathrm{C}(9)-\mathrm{C}(8)$ & $120.7(2)$ \\
\hline $\mathrm{C}(10)-\mathrm{C}(9)-\mathrm{H}(9)$ & 119.6 \\
\hline $\mathrm{C}(8)-\mathrm{C}(9)-\mathrm{H}(9)$ & 119.6 \\
\hline $\mathrm{C}(11)-\mathrm{C}(10)-\mathrm{C}(9)$ & $120.3(2)$ \\
\hline $\mathrm{C}(11)-\mathrm{C}(10)-\mathrm{H}(10)$ & 119.9 \\
\hline $\mathrm{C}(9)-\mathrm{C}(10)-\mathrm{H}(10)$ & 119.9 \\
\hline $\mathrm{C}(10)-\mathrm{C}(11)-\mathrm{C}(12)$ & $119.90(19)$ \\
\hline $\mathrm{C}(10)-\mathrm{C}(11)-\mathrm{H}(11)$ & 120.0 \\
\hline $\mathrm{C}(12)-\mathrm{C}(11)-\mathrm{H}(11)$ & 120.0 \\
\hline $\mathrm{C}(11)-\mathrm{C}(12)-\mathrm{C}(13)$ & $120.0(2)$ \\
\hline $\mathrm{C}(11)-\mathrm{C}(12)-\mathrm{H}(12)$ & 120.0 \\
\hline $\mathrm{C}(13)-\mathrm{C}(12)-\mathrm{H}(12)$ & 120.0 \\
\hline $\mathrm{C}(12)-\mathrm{C}(13)-\mathrm{C}(8)$ & $121.04(19)$ \\
\hline $\mathrm{C}(12)-\mathrm{C}(13)-\mathrm{H}(13)$ & 119.5 \\
\hline $\mathrm{C}(8)-\mathrm{C}(13)-\mathrm{H}(13)$ & 119.5 \\
\hline$C(15)-C(14)-C(19)$ & $118.6(2)$ \\
\hline $\mathrm{C}(15)-\mathrm{C}(14)-\mathrm{C}(1)$ & $119.45(18)$ \\
\hline $\mathrm{C}(19)-\mathrm{C}(14)-\mathrm{C}(1)$ & $121.9(2)$ \\
\hline$C(14)-C(15)-C(16)$ & 121.1(2) \\
\hline $\mathrm{C}(14)-\mathrm{C}(15)-\mathrm{H}(15)$ & 119.4 \\
\hline $\mathrm{C}(16)-\mathrm{C}(15)-\mathrm{H}(15)$ & 119.4 \\
\hline
\end{tabular}




\begin{tabular}{|c|c|}
\hline$C(15)-C(16)-C(17)$ & $119.6(2)$ \\
\hline $\mathrm{C}(15)-\mathrm{C}(16)-\mathrm{H}(16)$ & 120.2 \\
\hline $\mathrm{C}(17)-\mathrm{C}(16)-\mathrm{H}(16)$ & 120.2 \\
\hline $\mathrm{C}(18)-\mathrm{C}(17)-\mathrm{C}(16)$ & $119.8(2)$ \\
\hline $\mathrm{C}(18)-\mathrm{C}(17)-\mathrm{H}(17)$ & 120.1 \\
\hline $\mathrm{C}(16)-\mathrm{C}(17)-\mathrm{H}(17)$ & 120.1 \\
\hline $\mathrm{C}(17)-\mathrm{C}(18)-\mathrm{C}(19)$ & $120.7(2)$ \\
\hline $\mathrm{C}(17)-\mathrm{C}(18)-\mathrm{H}(18)$ & 119.7 \\
\hline $\mathrm{C}(19)-\mathrm{C}(18)-\mathrm{H}(18)$ & 119.7 \\
\hline$C(18)-C(19)-C(14)$ & $120.2(2)$ \\
\hline $\mathrm{C}(18)-\mathrm{C}(19)-\mathrm{H}(19)$ & 119.9 \\
\hline $\mathrm{C}(14)-\mathrm{C}(19)-\mathrm{H}(19)$ & 119.9 \\
\hline $\mathrm{N}(1)-\mathrm{C}(20)-\mathrm{C}(21)$ & $59.64(13)$ \\
\hline $\mathrm{N}(1)-\mathrm{C}(20)-\mathrm{H}(20 \mathrm{~A})$ & 117.8 \\
\hline $\mathrm{C}(21)-\mathrm{C}(20)-\mathrm{H}(20 \mathrm{~A})$ & 117.8 \\
\hline $\mathrm{N}(1)-\mathrm{C}(20)-\mathrm{H}(20 \mathrm{~B})$ & 117.8 \\
\hline $\mathrm{C}(21)-\mathrm{C}(20)-\mathrm{H}(20 \mathrm{~B})$ & 117.8 \\
\hline $\mathrm{H}(20 \mathrm{~A})-\mathrm{C}(20)-\mathrm{H}(20 \mathrm{~B})$ & 114.9 \\
\hline $\mathrm{N}(1)-\mathrm{C}(21)-\mathrm{C}(20)$ & $59.59(14)$ \\
\hline $\mathrm{N}(1)-\mathrm{C}(21)-\mathrm{C}(22)$ & $118.23(18)$ \\
\hline $\mathrm{C}(20)-\mathrm{C}(21)-\mathrm{C}(22)$ & $122.6(2)$ \\
\hline $\mathrm{N}(1)-\mathrm{C}(21)-\mathrm{H}(21)$ & 115.0 \\
\hline $\mathrm{C}(20)-\mathrm{C}(21)-\mathrm{H}(21)$ & 115.0 \\
\hline $\mathrm{C}(22)-\mathrm{C}(21)-\mathrm{H}(21)$ & 115.0 \\
\hline $\mathrm{N}(2)-\mathrm{C}(22)-\mathrm{C}(23)$ & $113.0(2)$ \\
\hline $\mathrm{N}(2)-\mathrm{C}(22)-\mathrm{C}(21)$ & $107.29(19)$ \\
\hline$C(23)-C(22)-C(21)$ & $115.5(2)$ \\
\hline $\mathrm{N}(2)-\mathrm{C}(22)-\mathrm{H}(22)$ & 106.9 \\
\hline $\mathrm{C}(23)-\mathrm{C}(22)-\mathrm{H}(22)$ & 106.9 \\
\hline $\mathrm{C}(21)-\mathrm{C}(22)-\mathrm{H}(22)$ & 106.9 \\
\hline $\mathrm{C}(22)-\mathrm{C}(23)-\mathrm{H}(23 \mathrm{~A})$ & 109.5 \\
\hline $\mathrm{C}(22)-\mathrm{C}(23)-\mathrm{H}(23 \mathrm{~B})$ & 109.5 \\
\hline $\mathrm{H}(23 \mathrm{~A})-\mathrm{C}(23)-\mathrm{H}(23 \mathrm{~B})$ & 109.5 \\
\hline $\mathrm{C}(22)-\mathrm{C}(23)-\mathrm{H}(23 \mathrm{C})$ & 109.5 \\
\hline $\mathrm{H}(23 \mathrm{~A})-\mathrm{C}(23)-\mathrm{H}(23 \mathrm{C})$ & 109.5 \\
\hline $\mathrm{H}(23 \mathrm{~B})-\mathrm{C}(23)-\mathrm{H}(23 \mathrm{C})$ & 109.5 \\
\hline
\end{tabular}


Symmetry transformations used to generate equivalent atoms: 
Table 4. Anisotropic displacement parameters $\left(\AA^{2} \times 10^{3}\right)$ for 26. The anisotropic displacement factor exponent takes the form: $-2 \pi^{2}\left[h^{2} a^{* 2} U^{11}+\ldots+2 h k a^{*} b^{*} U^{12}\right]$

\begin{tabular}{|c|c|c|c|c|c|c|}
\hline & $\mathrm{U}^{11}$ & $\mathrm{U}^{22}$ & $\mathrm{U}^{33}$ & $\mathrm{U}^{23}$ & $\mathrm{U}^{13}$ & $\mathrm{U}^{12}$ \\
\hline $\mathrm{N}(1)$ & $23(1)$ & $22(1)$ & $24(1)$ & $1(1)$ & $5(1)$ & $12(1)$ \\
\hline $\mathrm{N}(2)$ & $32(1)$ & $33(1)$ & $38(1)$ & $1(1)$ & $-6(1)$ & $14(1)$ \\
\hline $\mathrm{N}(3)$ & $29(1)$ & $39(1)$ & $29(1)$ & $-5(1)$ & $-4(1)$ & $18(1)$ \\
\hline $\mathrm{N}(4)$ & $35(1)$ & $37(1)$ & $35(1)$ & $1(1)$ & $1(1)$ & $14(1)$ \\
\hline $\mathrm{C}(1)$ & $18(1)$ & $18(1)$ & $24(1)$ & $2(1)$ & $3(1)$ & $10(1)$ \\
\hline$C(2)$ & $17(1)$ & $20(1)$ & 21(1) & $2(1)$ & $1(1)$ & $10(1)$ \\
\hline$C(3)$ & $23(1)$ & $24(1)$ & $22(1)$ & $-1(1)$ & $0(1)$ & $14(1)$ \\
\hline$C(4)$ & $28(1)$ & $37(1)$ & 21(1) & $3(1)$ & $4(1)$ & 20(1) \\
\hline$C(5)$ & $20(1)$ & $30(1)$ & $26(1)$ & $9(1)$ & $3(1)$ & 11(1) \\
\hline$C(6)$ & $21(1)$ & $22(1)$ & $30(1)$ & $3(1)$ & $-1(1)$ & $9(1)$ \\
\hline$C(7)$ & $20(1)$ & $21(1)$ & $26(1)$ & $1(1)$ & $1(1)$ & $10(1)$ \\
\hline $\mathrm{C}(8)$ & $22(1)$ & $18(1)$ & $22(1)$ & $1(1)$ & $2(1)$ & 11(1) \\
\hline$C(9)$ & $26(1)$ & $25(1)$ & $24(1)$ & $1(1)$ & $1(1)$ & $15(1)$ \\
\hline$C(10)$ & $36(1)$ & $32(1)$ & $30(1)$ & $2(1)$ & $2(1)$ & $25(1)$ \\
\hline $\mathrm{C}(11)$ & $45(1)$ & $25(1)$ & $27(1)$ & $1(1)$ & $2(1)$ & $24(1)$ \\
\hline$C(12)$ & $33(1)$ & $20(1)$ & $26(1)$ & $1(1)$ & $1(1)$ & $12(1)$ \\
\hline$C(13)$ & $24(1)$ & $22(1)$ & $23(1)$ & $2(1)$ & $2(1)$ & $13(1)$ \\
\hline$C(14)$ & $16(1)$ & $18(1)$ & $35(1)$ & $6(1)$ & $1(1)$ & $8(1)$ \\
\hline$C(15)$ & $25(1)$ & $26(1)$ & $32(1)$ & $7(1)$ & $-1(1)$ & $12(1)$ \\
\hline$C(16)$ & $29(1)$ & $26(1)$ & $41(1)$ & $5(1)$ & $-12(1)$ & $8(1)$ \\
\hline$C(17)$ & $21(1)$ & $29(1)$ & $63(2)$ & $8(1)$ & $-11(1)$ & $10(1)$ \\
\hline $\mathrm{C}(18)$ & $23(1)$ & $34(1)$ & $67(2)$ & $5(1)$ & $-2(1)$ & $16(1)$ \\
\hline$C(19)$ & $23(1)$ & $25(1)$ & $45(1)$ & $2(1)$ & $2(1)$ & $13(1)$ \\
\hline$C(20)$ & $34(1)$ & $24(1)$ & $29(1)$ & $4(1)$ & $13(1)$ & $11(1)$ \\
\hline$C(21)$ & $35(1)$ & $25(1)$ & 21(1) & $2(1)$ & $3(1)$ & $16(1)$ \\
\hline$C(22)$ & $40(1)$ & $32(1)$ & $21(1)$ & $1(1)$ & $-1(1)$ & $17(1)$ \\
\hline$C(23)$ & $43(1)$ & $33(1)$ & $30(1)$ & $-6(1)$ & $3(1)$ & $18(1)$ \\
\hline
\end{tabular}


Table 5. Hydrogen coordinates ( $\left.\times 10^{4}\right)$ and isotropic displacement parameters $\left(\AA^{2} \times 10^{3}\right)$ for 26 .

\begin{tabular}{|c|c|c|c|c|}
\hline & $\mathrm{x}$ & $\mathrm{y}$ & $\mathrm{z}$ & $\mathrm{U}(\mathrm{eq})$ \\
\hline $\mathrm{H}(3)$ & 5706 & 1398 & 9122 & 27 \\
\hline $\mathrm{H}(4)$ & 3410 & -632 & 9435 & 32 \\
\hline $\mathrm{H}(5)$ & 2187 & -3564 & 9319 & 31 \\
\hline $\mathrm{H}(6)$ & 3306 & -4452 & 8886 & 30 \\
\hline $\mathrm{H}(7)$ & 5609 & -2424 & 8571 & 27 \\
\hline $\mathrm{H}(9)$ & 5003 & 1842 & 8521 & 29 \\
\hline $\mathrm{H}(10)$ & 5170 & 4492 & 8433 & 35 \\
\hline $\mathrm{H}(11)$ & 7829 & 7006 & 8421 & 36 \\
\hline $\mathrm{H}(12)$ & 10346 & 6882 & 8493 & 32 \\
\hline $\mathrm{H}(13)$ & 10191 & 4248 & 8588 & 27 \\
\hline $\mathrm{H}(15)$ & 8761 & 2473 & 9196 & 34 \\
\hline $\mathrm{H}(16)$ & 11144 & 2857 & 9498 & 41 \\
\hline $\mathrm{H}(17)$ & 12876 & 1809 & 9287 & 47 \\
\hline $\mathrm{H}(18)$ & 12204 & 385 & 8780 & 48 \\
\hline $\mathrm{H}(19)$ & 9869 & 69 & 8470 & 37 \\
\hline $\mathrm{H}(20 \mathrm{~A})$ & 9047 & 830 & 7860 & 37 \\
\hline $\mathrm{H}(20 \mathrm{~B})$ & 9132 & 2538 & 8041 & 37 \\
\hline $\mathrm{H}(21)$ & 6094 & 1202 & 8033 & 32 \\
\hline $\mathrm{H}(22)$ & 5338 & -666 & 7561 & 37 \\
\hline $\mathrm{H}(23 \mathrm{~A})$ & 6533 & -2616 & 7907 & 53 \\
\hline $\mathrm{H}(23 \mathrm{~B})$ & 7313 & -1577 & 7569 & 53 \\
\hline $\mathrm{H}(23 \mathrm{C})$ & 5436 & -3238 & 7572 & 53 \\
\hline
\end{tabular}




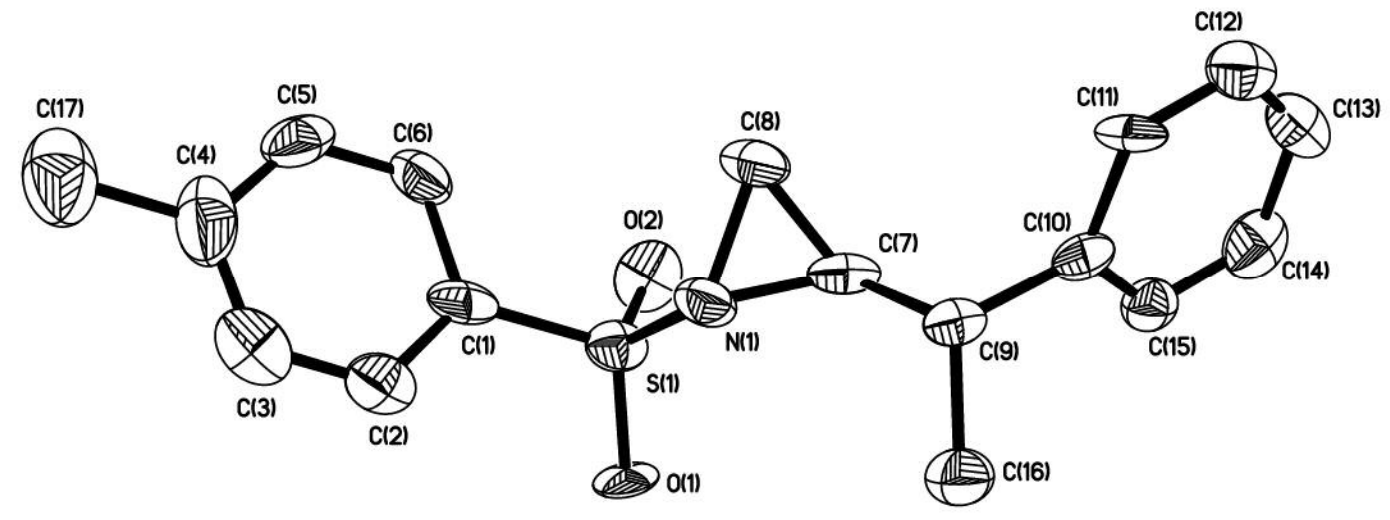

ORTEP of compound 27 
Table 1. Crystal data and structure refinement for 27.

Identification code

Empirical formula

Formula weight

Temperature

Wavelength

Crystal system

Space group

Unit cell dimensions

Volume

$\mathrm{Z}$

Density (calculated)

Absorption coefficient

$\mathrm{F}(000)$

Crystal size

Theta range for data collection

Index ranges

Reflections collected

Independent reflections

Completeness to theta $=24.99^{\circ}$

Absorption correction

Max. and min. transmission

Refinement method

Data / restraints / parameters

Goodness-of-fit on $\mathrm{F}^{2}$

Final $\mathrm{R}$ indices $[\mathrm{I}>2 \operatorname{sigma}(\mathrm{I})]$

$\mathrm{R}$ indices (all data)

Absolute structure parameter

Largest diff. peak and hole ohj136_0m

C17 H19 N O2 S

301.39

296(2) K

$0.71073 \AA$

Monoclinic

$\mathrm{P} 2_{1}$

$\mathrm{a}=6.2669(17) \AA$

$\alpha=90^{\circ}$.

$\mathrm{b}=7.288(2) \AA$

$\beta=92.276(19)^{\circ}$.

$\mathrm{c}=17.483(6) \AA$

$\gamma=90^{\circ}$.

797.9(4) $\AA^{3}$

2

$1.254 \mathrm{Mg} / \mathrm{m}^{3}$

$0.207 \mathrm{~mm}^{-1}$

320

$0.11 \times 0.03 \times 0.03 \mathrm{~mm}^{3}$

1.17 to $24.99^{\circ}$.

$-7<=\mathrm{h}<=7,-8<=\mathrm{k}<=6,-20<=\mathrm{l}<=20$

4006

$1521[\mathrm{R}(\mathrm{int})=0.1144]$

$99.2 \%$

Semi-empirical from equivalents

0.9938 and 0.9776

Full-matrix least-squares on $\mathrm{F}^{2}$

$1521 / 1 / 193$

1.070

$\mathrm{R} 1=0.0977, \mathrm{wR} 2=0.2336$

$\mathrm{R} 1=0.1440, \mathrm{wR} 2=0.2661$

$0(10)$

0.692 and -0.473 e. $\AA^{-3}$ 
Table 2. Atomic coordinates ( x 10 $0^{4}$ ) and equivalent isotropic displacement parameters $\left(\AA^{2} \times 10^{3}\right)$ for $27 . \mathrm{U}(\mathrm{eq})$ is defined as one third of the trace of the orthogonalized $\mathrm{U}^{\mathrm{ij}}$ tensor.

\begin{tabular}{|c|c|c|c|c|}
\hline & $\mathrm{x}$ & $\mathrm{y}$ & $\mathrm{z}$ & $\mathrm{U}(\mathrm{eq})$ \\
\hline$S(1)$ & 4736(4) & 2198(4) & $7939(2)$ & $36(1)$ \\
\hline $\mathrm{N}(1)$ & $3911(15)$ & $4314(15)$ & $7709(5)$ & $39(3)$ \\
\hline $\mathrm{O}(1)$ & $7019(10)$ & $2269(14)$ & 7982(4) & $45(2)$ \\
\hline $\mathrm{O}(2)$ & $3732(15)$ & $833(12)$ & $7450(5)$ & $52(2)$ \\
\hline $\mathrm{C}(1)$ & $3753(15)$ & 1997(18) & $8855(6)$ & $38(3)$ \\
\hline $\mathrm{C}(2)$ & $4870(20)$ & $2587(16)$ & 9494(7) & $45(3)$ \\
\hline $\mathrm{C}(3)$ & $4070(20)$ & $2500(20)$ & $10204(7)$ & $52(4)$ \\
\hline $\mathrm{C}(4)$ & $2050(30)$ & $1800(20)$ & $10317(7)$ & $68(5)$ \\
\hline $\mathrm{C}(5)$ & $890(20)$ & $1200(20)$ & $9656(8)$ & $53(4)$ \\
\hline$C(6)$ & 1647(19) & 1267(19) & $8932(6)$ & $45(3)$ \\
\hline$C(7)$ & $3630(16)$ & $4667(18)$ & $6870(7)$ & $38(3)$ \\
\hline $\mathrm{C}(8)$ & 1711(19) & $4470(20)$ & 7334(7) & $50(3)$ \\
\hline $\mathrm{C}(9)$ & $4385(16)$ & $6516(14)$ & $6587(6)$ & $28(2)$ \\
\hline $\mathrm{C}(10)$ & $3519(17)$ & $6770(16)$ & $5771(6)$ & $35(3)$ \\
\hline $\mathrm{C}(11)$ & $1566(16)$ & 7673(17) & $5618(6)$ & $37(3)$ \\
\hline $\mathrm{C}(12)$ & $710(20)$ & 7797(17) & $4886(7)$ & $43(3)$ \\
\hline $\mathrm{C}(13)$ & 1694(19) & $7008(19)$ & $4288(7)$ & $43(3)$ \\
\hline$C(14)$ & $3670(20)$ & $6140(18)$ & $4405(7)$ & $48(3)$ \\
\hline$C(15)$ & $4523(18)$ & $6004(16)$ & $5149(6)$ & $36(3)$ \\
\hline$C(16)$ & $6820(17)$ & $6680(20)$ & $6685(7)$ & $47(3)$ \\
\hline$C(17)$ & $1180(30)$ & $1640(30)$ & $11107(8)$ & $81(6)$ \\
\hline
\end{tabular}


Table 3. Bond lengths [ $\left[\AA\right.$ ] and angles $\left[{ }^{\circ}\right]$ for 27.

\begin{tabular}{|c|c|}
\hline $\mathrm{S}(1)-\mathrm{O}(1)$ & $1.431(7)$ \\
\hline $\mathrm{S}(1)-\mathrm{O}(2)$ & $1.440(9)$ \\
\hline $\mathrm{S}(1)-\mathrm{N}(1)$ & $1.670(11)$ \\
\hline$S(1)-C(1)$ & $1.746(11)$ \\
\hline $\mathrm{N}(1)-\mathrm{C}(7)$ & $1.492(15)$ \\
\hline $\mathrm{N}(1)-\mathrm{C}(8)$ & $1.508(15)$ \\
\hline$C(1)-C(2)$ & $1.365(15)$ \\
\hline$C(1)-C(6)$ & $1.434(15)$ \\
\hline$C(2)-C(3)$ & $1.359(16)$ \\
\hline $\mathrm{C}(2)-\mathrm{H}(2)$ & 0.9300 \\
\hline $\mathrm{C}(3)-\mathrm{C}(4)$ & $1.39(2)$ \\
\hline $\mathrm{C}(3)-\mathrm{H}(3)$ & 0.9300 \\
\hline $\mathrm{C}(4)-\mathrm{C}(5)$ & $1.41(2)$ \\
\hline $\mathrm{C}(4)-\mathrm{C}(17)$ & $1.508(18)$ \\
\hline $\mathrm{C}(5)-\mathrm{C}(6)$ & $1.368(16)$ \\
\hline $\mathrm{C}(5)-\mathrm{H}(5)$ & 0.9300 \\
\hline $\mathrm{C}(6)-\mathrm{H}(6)$ & 0.9300 \\
\hline$C(7)-C(8)$ & $1.484(16)$ \\
\hline $\mathrm{C}(7)-\mathrm{C}(9)$ & $1.518(16)$ \\
\hline $\mathrm{C}(7)-\mathrm{H}(7)$ & 0.9800 \\
\hline $\mathrm{C}(8)-\mathrm{H}(8 \mathrm{~A})$ & 0.9700 \\
\hline $\mathrm{C}(8)-\mathrm{H}(8 \mathrm{~B})$ & 0.9700 \\
\hline $\mathrm{C}(9)-\mathrm{C}(10)$ & $1.518(15)$ \\
\hline $\mathrm{C}(9)-\mathrm{C}(16)$ & $1.533(14)$ \\
\hline $\mathrm{C}(9)-\mathrm{H}(9)$ & 0.9800 \\
\hline$C(10)-C(15)$ & $1.395(15)$ \\
\hline $\mathrm{C}(10)-\mathrm{C}(11)$ & $1.406(15)$ \\
\hline $\mathrm{C}(11)-\mathrm{C}(12)$ & $1.373(16)$ \\
\hline $\mathrm{C}(11)-\mathrm{H}(11)$ & 0.9300 \\
\hline$C(12)-C(13)$ & $1.363(17)$ \\
\hline $\mathrm{C}(12)-\mathrm{H}(12)$ & 0.9300 \\
\hline $\mathrm{C}(13)-\mathrm{C}(14)$ & $1.398(17)$ \\
\hline $\mathrm{C}(13)-\mathrm{H}(13)$ & 0.9300 \\
\hline $\mathrm{C}(14)-\mathrm{C}(15)$ & $1.389(17)$ \\
\hline
\end{tabular}




\begin{tabular}{|c|c|}
\hline $\mathrm{C}(14)-\mathrm{H}(14)$ & 0.9300 \\
\hline $\mathrm{C}(15)-\mathrm{H}(15)$ & 0.9300 \\
\hline $\mathrm{C}(16)-\mathrm{H}(16 \mathrm{~A})$ & 0.9600 \\
\hline $\mathrm{C}(16)-\mathrm{H}(16 \mathrm{~B})$ & 0.9600 \\
\hline $\mathrm{C}(16)-\mathrm{H}(16 \mathrm{C})$ & 0.9600 \\
\hline $\mathrm{C}(17)-\mathrm{H}(17 \mathrm{~A})$ & 0.9600 \\
\hline $\mathrm{C}(17)-\mathrm{H}(17 \mathrm{~B})$ & 0.9600 \\
\hline $\mathrm{C}(17)-\mathrm{H}(17 \mathrm{C})$ & 0.9600 \\
\hline $\mathrm{O}(1)-\mathrm{S}(1)-\mathrm{O}(2)$ & $117.9(6)$ \\
\hline $\mathrm{O}(1)-\mathrm{S}(1)-\mathrm{N}(1)$ & $106.2(5)$ \\
\hline $\mathrm{O}(2)-\mathrm{S}(1)-\mathrm{N}(1)$ & $111.8(6)$ \\
\hline $\mathrm{O}(1)-\mathrm{S}(1)-\mathrm{C}(1)$ & $110.1(5)$ \\
\hline $\mathrm{O}(2)-\mathrm{S}(1)-\mathrm{C}(1)$ & $109.0(6)$ \\
\hline $\mathrm{N}(1)-\mathrm{S}(1)-\mathrm{C}(1)$ & $100.4(5)$ \\
\hline $\mathrm{C}(7)-\mathrm{N}(1)-\mathrm{C}(8)$ & $59.3(7)$ \\
\hline $\mathrm{C}(7)-\mathrm{N}(1)-\mathrm{S}(1)$ & $114.7(8)$ \\
\hline C(8)-N(1)-S(1) & $116.2(9)$ \\
\hline$C(2)-C(1)-C(6)$ & 119.1(10) \\
\hline $\mathrm{C}(2)-\mathrm{C}(1)-\mathrm{S}(1)$ & $122.4(9)$ \\
\hline$C(6)-C(1)-S(1)$ & $118.5(8)$ \\
\hline $\mathrm{C}(3)-\mathrm{C}(2)-\mathrm{C}(1)$ & $122.3(12)$ \\
\hline $\mathrm{C}(3)-\mathrm{C}(2)-\mathrm{H}(2)$ & 118.8 \\
\hline $\mathrm{C}(1)-\mathrm{C}(2)-\mathrm{H}(2)$ & 118.8 \\
\hline $\mathrm{C}(2)-\mathrm{C}(3)-\mathrm{C}(4)$ & $121.4(12)$ \\
\hline $\mathrm{C}(2)-\mathrm{C}(3)-\mathrm{H}(3)$ & 119.3 \\
\hline $\mathrm{C}(4)-\mathrm{C}(3)-\mathrm{H}(3)$ & 119.3 \\
\hline $\mathrm{C}(3)-\mathrm{C}(4)-\mathrm{C}(5)$ & $116.0(11)$ \\
\hline $\mathrm{C}(3)-\mathrm{C}(4)-\mathrm{C}(17)$ & $121.6(14)$ \\
\hline $\mathrm{C}(5)-\mathrm{C}(4)-\mathrm{C}(17)$ & $122.4(15)$ \\
\hline$C(6)-C(5)-C(4)$ & $124.3(12)$ \\
\hline $\mathrm{C}(6)-\mathrm{C}(5)-\mathrm{H}(5)$ & 117.9 \\
\hline $\mathrm{C}(4)-\mathrm{C}(5)-\mathrm{H}(5)$ & 117.9 \\
\hline$C(5)-C(6)-C(1)$ & $116.9(11)$ \\
\hline $\mathrm{C}(5)-\mathrm{C}(6)-\mathrm{H}(6)$ & 121.6 \\
\hline $\mathrm{C}(1)-\mathrm{C}(6)-\mathrm{H}(6)$ & 121.6 \\
\hline
\end{tabular}




\begin{tabular}{|c|c|}
\hline $\mathrm{C}(8)-\mathrm{C}(7)-\mathrm{N}(1)$ & $60.9(7)$ \\
\hline $\mathrm{C}(8)-\mathrm{C}(7)-\mathrm{C}(9)$ & $122.4(11)$ \\
\hline $\mathrm{N}(1)-\mathrm{C}(7)-\mathrm{C}(9)$ & $116.6(10)$ \\
\hline $\mathrm{C}(8)-\mathrm{C}(7)-\mathrm{H}(7)$ & 115.3 \\
\hline $\mathrm{N}(1)-\mathrm{C}(7)-\mathrm{H}(7)$ & 115.3 \\
\hline $\mathrm{C}(9)-\mathrm{C}(7)-\mathrm{H}(7)$ & 115.3 \\
\hline $\mathrm{C}(7)-\mathrm{C}(8)-\mathrm{N}(1)$ & $59.8(7)$ \\
\hline $\mathrm{C}(7)-\mathrm{C}(8)-\mathrm{H}(8 \mathrm{~A})$ & 117.8 \\
\hline $\mathrm{N}(1)-\mathrm{C}(8)-\mathrm{H}(8 \mathrm{~A})$ & 117.8 \\
\hline $\mathrm{C}(7)-\mathrm{C}(8)-\mathrm{H}(8 \mathrm{~B})$ & 117.8 \\
\hline $\mathrm{N}(1)-\mathrm{C}(8)-\mathrm{H}(8 \mathrm{~B})$ & 117.8 \\
\hline $\mathrm{H}(8 \mathrm{~A})-\mathrm{C}(8)-\mathrm{H}(8 \mathrm{~B})$ & 114.9 \\
\hline$C(7)-C(9)-C(10)$ & $108.1(9)$ \\
\hline$C(7)-C(9)-C(16)$ & $110.7(9)$ \\
\hline $\mathrm{C}(10)-\mathrm{C}(9)-\mathrm{C}(16)$ & $114.4(8)$ \\
\hline $\mathrm{C}(7)-\mathrm{C}(9)-\mathrm{H}(9)$ & 107.8 \\
\hline $\mathrm{C}(10)-\mathrm{C}(9)-\mathrm{H}(9)$ & 107.8 \\
\hline $\mathrm{C}(16)-\mathrm{C}(9)-\mathrm{H}(9)$ & 107.8 \\
\hline$C(15)-C(10)-C(11)$ & 117.1(10) \\
\hline $\mathrm{C}(15)-\mathrm{C}(10)-\mathrm{C}(9)$ & $121.9(10)$ \\
\hline $\mathrm{C}(11)-\mathrm{C}(10)-\mathrm{C}(9)$ & $120.8(9)$ \\
\hline $\mathrm{C}(12)-\mathrm{C}(11)-\mathrm{C}(10)$ & $120.9(10)$ \\
\hline $\mathrm{C}(12)-\mathrm{C}(11)-\mathrm{H}(11)$ & 119.5 \\
\hline $\mathrm{C}(10)-\mathrm{C}(11)-\mathrm{H}(11)$ & 119.5 \\
\hline $\mathrm{C}(13)-\mathrm{C}(12)-\mathrm{C}(11)$ & $120.9(12)$ \\
\hline $\mathrm{C}(13)-\mathrm{C}(12)-\mathrm{H}(12)$ & 119.5 \\
\hline $\mathrm{C}(11)-\mathrm{C}(12)-\mathrm{H}(12)$ & 119.5 \\
\hline $\mathrm{C}(12)-\mathrm{C}(13)-\mathrm{C}(14)$ & $120.4(11)$ \\
\hline $\mathrm{C}(12)-\mathrm{C}(13)-\mathrm{H}(13)$ & 119.8 \\
\hline $\mathrm{C}(14)-\mathrm{C}(13)-\mathrm{H}(13)$ & 119.8 \\
\hline$C(15)-C(14)-C(13)$ & $118.4(11)$ \\
\hline $\mathrm{C}(15)-\mathrm{C}(14)-\mathrm{H}(14)$ & 120.8 \\
\hline $\mathrm{C}(13)-\mathrm{C}(14)-\mathrm{H}(14)$ & 120.8 \\
\hline$C(14)-C(15)-C(10)$ & $122.1(11)$ \\
\hline $\mathrm{C}(14)-\mathrm{C}(15)-\mathrm{H}(15)$ & 118.9 \\
\hline $\mathrm{C}(10)-\mathrm{C}(15)-\mathrm{H}(15)$ & 118.9 \\
\hline
\end{tabular}




$\begin{array}{ll}\text { C(9)-C(16)-H(16A) } & 109.5 \\ \text { C(9)-C(16)-H(16B) } & 109.5 \\ \text { H(16A)-C(16)-H(16B) } & 109.5 \\ \text { C(9)-C(16)-H(16C) } & 109.5 \\ \text { H(16A)-C(16)-H(16C) } & 109.5 \\ \text { H(16B)-C(16)-H(16C) } & 109.5 \\ \text { C(4)-C(17)-H(17A) } & 109.5 \\ \text { C(4)-C(17)-H(17B) } & 109.5 \\ \text { H(17A)-C(17)-H(17B) } & 109.5 \\ \text { C(4)-C(17)-H(17C) } & 109.5 \\ \text { H(17A)-C(17)-H(17C) } & 109.5 \\ \text { H(17B)-C(17)-H(17C) } & 109.5\end{array}$

Symmetry transformations used to generate equivalent atoms: 
Table 4. Anisotropic displacement parameters $\left(\AA^{2} \times 10^{3}\right)$ for 27. The anisotropic displacement factor exponent takes the form: $-2 \pi^{2}\left[h^{2} a^{* 2} U^{11}+\ldots+2 h k a^{*} b^{*} U^{12}\right]$

\begin{tabular}{|c|c|c|c|c|c|c|}
\hline & $\mathrm{U}^{11}$ & $\mathrm{U}^{22}$ & $\mathrm{U}^{33}$ & $\mathrm{U}^{23}$ & $\mathrm{U}^{13}$ & $\mathrm{U}^{12}$ \\
\hline$S(1)$ & $31(1)$ & $27(1)$ & $51(2)$ & $3(2)$ & $-2(1)$ & $4(2)$ \\
\hline $\mathrm{N}(1)$ & $29(6)$ & $43(7)$ & $43(5)$ & $4(4)$ & $-12(4)$ & $-7(5)$ \\
\hline $\mathrm{O}(1)$ & $10(3)$ & $52(5)$ & $73(5)$ & $25(5)$ & $6(3)$ & $10(5)$ \\
\hline $\mathrm{O}(2)$ & $66(7)$ & $35(6)$ & $57(5)$ & $-8(4)$ & $9(4)$ & $-4(5)$ \\
\hline $\mathrm{C}(1)$ & $23(5)$ & $28(7)$ & $61(7)$ & $4(6)$ & $-9(5)$ & $9(6)$ \\
\hline$C(2)$ & $44(7)$ & $29(8)$ & $60(7)$ & $8(6)$ & $-12(6)$ & $-12(6)$ \\
\hline$C(3)$ & $66(9)$ & $33(9)$ & $56(7)$ & $-7(6)$ & $-15(6)$ & $0(7)$ \\
\hline $\mathrm{C}(4)$ & $85(11)$ & $74(14)$ & $45(7)$ & $15(7)$ & $10(7)$ & $24(10)$ \\
\hline$C(5)$ & $28(7)$ & $54(9)$ & $77(9)$ & $24(7)$ & $13(6)$ & 11(6) \\
\hline$C(6)$ & $38(7)$ & $56(9)$ & $41(6)$ & $2(6)$ & $-15(5)$ & $-4(6)$ \\
\hline$C(7)$ & $19(6)$ & $39(7)$ & $56(7)$ & $-7(6)$ & $-3(5)$ & $-3(5)$ \\
\hline $\mathrm{C}(8)$ & $25(6)$ & $62(10)$ & $62(8)$ & $14(7)$ & $-10(5)$ & $-1(6)$ \\
\hline $\mathrm{C}(9)$ & $22(6)$ & $17(5)$ & $46(6)$ & $-4(4)$ & $3(4)$ & $1(4)$ \\
\hline$C(10)$ & $26(6)$ & $34(8)$ & $45(6)$ & $0(5)$ & $0(4)$ & $-16(5)$ \\
\hline$C(11)$ & $14(5)$ & 49(9) & $48(6)$ & $-6(5)$ & $-1(4)$ & $5(5)$ \\
\hline$C(12)$ & $36(7)$ & $29(7)$ & $64(8)$ & $2(5)$ & $-4(6)$ & $0(5)$ \\
\hline$C(13)$ & $49(7)$ & $22(7)$ & $57(7)$ & $1(6)$ & $-21(5)$ & $-6(7)$ \\
\hline$C(14)$ & $55(9)$ & $27(8)$ & $61(8)$ & $-1(6)$ & $13(6)$ & $-4(6)$ \\
\hline$C(15)$ & $32(7)$ & $29(7)$ & $47(6)$ & $5(5)$ & $2(5)$ & $-2(5)$ \\
\hline$C(16)$ & $32(7)$ & $51(10)$ & $59(7)$ & $10(6)$ & $1(5)$ & $1(6)$ \\
\hline $\mathrm{C}(17)$ & $98(13)$ & $84(14)$ & $61(9)$ & $15(8)$ & $8(8)$ & $35(11)$ \\
\hline
\end{tabular}


Table 5. Hydrogen coordinates ( $\left.\times 10^{4}\right)$ and isotropic displacement parameters $\left(\AA^{2} \times 10^{3}\right)$ for 27.

\begin{tabular}{|c|c|c|c|c|}
\hline & $\mathrm{x}$ & $\mathrm{y}$ & $\mathrm{z}$ & $\mathrm{U}(\mathrm{eq})$ \\
\hline $\mathrm{H}(2)$ & 6236 & 3067 & 9442 & 54 \\
\hline $\mathrm{H}(3)$ & 4894 & 2917 & 10623 & 63 \\
\hline $\mathrm{H}(5)$ & -469 & 730 & 9715 & 63 \\
\hline $\mathrm{H}(6)$ & 832 & 858 & 8510 & 54 \\
\hline $\mathrm{H}(7)$ & 3937 & 3621 & 6540 & 46 \\
\hline $\mathrm{H}(8 \mathrm{~A})$ & 877 & 3351 & 7272 & 60 \\
\hline $\mathrm{H}(8 \mathrm{~B})$ & 887 & 5561 & 7434 & 60 \\
\hline $\mathrm{H}(9)$ & 3752 & 7467 & 6903 & 34 \\
\hline $\mathrm{H}(11)$ & 847 & 8194 & 6020 & 44 \\
\hline $\mathrm{H}(12)$ & -568 & 8429 & 4796 & 52 \\
\hline $\mathrm{H}(13)$ & 1050 & 7047 & 3800 & 52 \\
\hline $\mathrm{H}(14)$ & 4393 & 5666 & 3995 & 57 \\
\hline $\mathrm{H}(15)$ & 5806 & 5381 & 5234 & 43 \\
\hline $\mathrm{H}(16 \mathrm{~A})$ & 7218 & 6765 & 7219 & 71 \\
\hline $\mathrm{H}(16 \mathrm{~B})$ & 7290 & 7758 & 6425 & 71 \\
\hline $\mathrm{H}(16 \mathrm{C})$ & 7476 & 5616 & 6471 & 71 \\
\hline $\mathrm{H}(17 \mathrm{~A})$ & 1456 & 432 & 11305 & 121 \\
\hline $\mathrm{H}(17 \mathrm{~B})$ & -332 & 1859 & 11080 & 121 \\
\hline $\mathrm{H}(17 \mathrm{C})$ & 1862 & 2532 & 11439 & 121 \\
\hline
\end{tabular}

